Ann. Scient. Éc. Norm. Sup.,

$4^{\mathrm{e}}$ série, t. 40, 2007, p. 765 à 813.

\title{
BOUNDARY BEHAVIOUR FOR $p$ HARMONIC FUNCTIONS IN LIPSCHITZ AND STARLIKE LIPSCHITZ RING DOMAINS
}

\author{
BY JOHN L. LEWIS ${ }^{1}$ AND KAJ NYSTRÖM
}

\begin{abstract}
In this paper we prove new results for $p$ harmonic functions, $p \neq 2,1<p<\infty$, in Lipschitz and starlike Lipschitz ring domains. In particular we prove the boundary Harnack inequality, Theorem 1, for the ratio of two positive $p$ harmonic functions vanishing on a portion of the boundary of a Lipschitz domain, with constants only depending on $p, n$ and the Lipschitz constant of the domain. For $p$ capacitary functions, in starlike Lipschitz ring domains, we prove an even stronger result, Theorem 2, showing that the ratio is Hölder continuous up to the boundary. Moreover, for $p$ capacitary functions in starlike Lipschitz ring domains we prove, Theorems 3 and 4, appropriate extensions to $p \neq 2,1<p<\infty$, of famous results of Dahlberg [12] and Jerison and Kenig [25] on the Poisson kernel associated to the Laplace operator (i.e. $p=2$ ).
\end{abstract}

(c) 2007 Elsevier Masson SAS

RÉSUMÉ. - Dans cet article, nous présentons de nouveaux résultats pour des fonctions $p$-harmoniques, $p \neq 2,1<p<\infty$, dans des domaines annulaires lipschitziens et lipschitziens étoilés. En particulier, nous démontrons l'inégalité de Harnack au bord (Théorème 1) pour le rapport de deux fonctions $p$-harmoniques strictement positives quand les deux fonctions s'annulent sur une partie du bord d'un domaine lipschitzien, avec constantes ne dépendant que de $p$, de $n$ et de la constante de Lipschitz. Pour les fonctions $p$-harmoniques de capacité, dans des domaines annulaires lipschitziens étoilés, nous prouvons un résultat encore plus fort (Théorème 2) démontrant que le rapport est Hölder continu jusqu'au bord. De plus, pour les fonctions $p$-harmoniques de capacité dans des domaines annulaires lipschitziens étoilés, nous montrons (Théorèmes 3 et 4 ) des extensions appropriées pour $p \neq 2,1<p<\infty$, de résultats très connus de Dahlberg [12] et de Jerison et Kenig [25] sur le noyau de Poisson associé à l'opérateur de Laplace (pour $p=2$ ).

(C) 2007 Elsevier Masson SAS

\section{Introduction}

In this paper we prove a number of new results concerning the boundary behaviour of $p$ capacitary functions, $p \neq 2$ and $1<p<\infty$, in starlike Lipschitz ring domains. Using our results we are also able to prove the boundary Harnack inequality for the ratio of two positive $p$ harmonic functions, vanishing on a portion of the boundary of a bounded Lipschitz domain $\Omega \subset \mathbb{R}^{n}$. The constants in the inequality only depend on $p, n$ and the Lipschitz constant of $\Omega$. To put these results into perspective we note that the boundary Harnack inequality for harmonic functions (i.e. $p=2$ ) in a Lipschitz domain was first introduced in [26] and later proved

\footnotetext{
${ }^{1}$ Lewis was partially supported by an NSF grant. 
independently by $[4,12,44]$. This inequality was generalized in [24], for $p=2$, to nontangentially accessible domains (NTA domains). In these settings it was also proved that the ratio of two positive harmonic functions, vanishing on a portion of the boundary, is Hölder continuous up to the boundary. The importance of these two results-the boundary Harnack inequality and Hölder continuity up to the boundary for quotients of harmonic functions- to potential theory, boundary value problems and free boundary problems in Lipschitz domains and beyond, for the Laplace operator and more general elliptic second order operators, can hardly be overstated. To be specific concerning areas where the above results are crucial we mention work of B. Dahlberg [12] as well as Jerison and Kenig [25] on harmonic measure and the Poisson kernel in Lipschitz and $C^{1}$ domains, the program of Caffarelli [7-9] for the analysis of elliptic free boundary problems and the program carried out in the papers $[3,23,27-30]$, on free boundary regularity and regularity of the Poisson kernel below the continuous threshold.

Analogues of these results for the $p$ Laplacian are easily stated but until now their proofs have eluded the experts, primarily because this operator is nonlinear when $p \neq 2$. In fact the results and techniques of this paper define a starting point for far reaching developments concerning the $p$ Laplace operator in Lipschitz domains and beyond. In this paper, which is the first in a sequel, we lay the groundwork for further developments by proving for $p$ capacitary functions in starlike Lipschitz ring domains: (a) the boundary Harnack inequality and Hölder continuity of quotients up to the boundary (Theorem 2), and (b) analogues of results of Dahlberg [12] (Theorem 3) and Jerison and Kenig [24] (Theorem 4). The boundary Harnack inequality is then (Theorem 1), extended to general bounded Lipschitz domains and to general positive $p$ harmonic functions vanishing on a portion of the boundary through comparison with appropriate $p$ capacitary functions. Hence an in-depth analysis of $p$ capacitary functions in starlike Lipschitz ring domains is the main focus of this paper.

To proceed and to state our results we need to introduce some notation. Points in Euclidean $n$ space $\mathbb{R}^{n}$ are denoted by $x=\left(x_{1}, \ldots, x_{n}\right)$ or $\left(x^{\prime}, x_{n}\right)$ where $x^{\prime}=\left(x_{1}, \ldots, x_{n-1}\right) \in \mathbb{R}^{n-1}$. We let $\bar{E}, \partial E$, diam $E$, be the closure, boundary, diameter, of the set $E \subset \mathbb{R}^{n}$ and we define $d(y, E)$ to equal the distance from $y \in \mathbb{R}^{n}$ to $E .\langle\cdot, \cdot\rangle$ denotes the standard inner product on $\mathbb{R}^{n}$ and we let $|x|=\langle x, x\rangle^{1 / 2}$ be the Euclidean norm of $x . B(x, r)=\left\{y \in \mathbb{R}^{n}:|x-y|<r\right\}$ is defined whenever $x \in \mathbb{R}^{n}, r>0$, and $d x$ denotes Lebesgue $n$ measure on $\mathbb{R}^{n}$. If $O \subset \mathbb{R}^{n}$ is open and $1 \leqslant q \leqslant \infty$, then by $W^{1, q}(O)$, we denote the space of equivalence classes of functions $f$ with distributional gradient $\nabla f=\left(f_{x_{1}}, \ldots, f_{x_{n}}\right)$, both of which are $q$-th power integrable on $O$. Let $\|f\|_{1, q}=\|f\|_{q}+\||\nabla f|\|_{q}$ be the norm in $W^{1, q}(O)$ where $\|\cdot\|_{q}$ denotes the usual Lebesgue $q$ norm in $O$. Next let $C_{0}^{\infty}(O)$ be infinitely differentiable functions with compact support in $O$ and let $W_{0}^{1, q}(O)$ be the closure of $C_{0}^{\infty}(O)$ in the norm of $W^{1, q}(O)$.

Given $G$ a bounded domain (i.e., a connected open set) and $1<p<\infty$, we say that $\hat{u}$ is $p$ harmonic in $G$ provided $\hat{u} \in W^{1, p}(G)$ and

$$
\int|\nabla \hat{u}|^{p-2}\langle\nabla \hat{u}, \nabla \theta\rangle d x=0
$$

whenever $\theta \in W_{0}^{1, p}(G)$. Observe that if $\hat{u}$ is smooth and $\nabla \hat{u} \neq 0$ in $G$, then

$$
\nabla \cdot\left(|\nabla \hat{u}|^{p-2} \nabla \hat{u}\right) \equiv 0 \quad \text { in } G
$$

so $\hat{u}$ is a classical solution in $G$ to the $p$ Laplace partial differential equation. Here, as in the sequel, $\nabla \cdot$ is the divergence operator. We note that $\phi: E \rightarrow \mathbb{R}$ is said to be Lipschitz on $E$ provided there exists $b, 0<b<\infty$, such that

$4^{\mathrm{e}}$ SÉRIE - TOME $40-2007-\mathrm{N}^{\circ} 5$ 


$$
|\phi(z)-\phi(w)| \leqslant b|z-w| \quad \text { whenever } z, w \in E .
$$

The infimum of all $b$ such that (1.3) holds is called the Lipschitz norm of $\phi$ on $E$, denoted $\| \phi \hat{\|}_{E}$. It is well known that if $E=\mathbb{R}^{n-1}$, then $\phi$ is differentiable on $\mathbb{R}^{n-1}$ and $\|\phi\|_{\mathbb{R}^{n-1}}=\|\mid \nabla \phi\|_{\infty}$.

Finally let $e_{i}, 1 \leqslant i \leqslant n$, denote the point in $\mathbb{R}^{n}$ with one in the $i$-th coordinate position and zeroes elsewhere. We now formulate our result on the boundary Harnack inequality for positive $p$ harmonic functions in Lipschitz domains.

THEOREM 1. - Let $G=\left\{y=\left(y^{\prime}, y_{n}\right) \in \mathbb{R}^{n}: y_{n}>\phi\left(y^{\prime}\right)\right\}$ where $\phi$ is Lipschitz on $\mathbb{R}^{n-1}$. Given $p, 1<p<\infty, w=\left(w^{\prime}, \phi\left(w^{\prime}\right)\right) \in \partial G$, and $r>0$, suppose that $\tilde{u}, \tilde{v}$ are positive $p$ harmonic functions in $G \cap B(w, r)$, that $\tilde{u}, \tilde{v}$ are continuous in $\bar{G} \cap B(w, r), \tilde{u}\left(w+\frac{r}{4} e_{n}\right)=\tilde{v}\left(w+\frac{r}{4} e_{n}\right)=1$ and that $\tilde{u}, \tilde{v}=0$ on $\partial G \cap B(w, r)$. Then there exists $c_{1}, 1 \leqslant c_{1}<\infty$, depending only on $p, n$, and $\||\nabla \phi|\|_{\infty}$ such that

$$
\frac{\tilde{u}(y)}{\tilde{v}(y)} \leqslant c_{1} \quad \text { whenever } y \in G \cap B\left(w, r / c_{1}\right) .
$$

The conclusion of Theorem 1 is known as a boundary Harnack inequality and as mentioned above the boundary Harnack inequality for harmonic functions (i.e. $p=2$ ) in a Lipschitz domain was first introduced in [26], later proved independently by $[4,12,44]$, and generalized in [24] to NTA-domains. For $p \neq 2$, and $\phi$ sufficiently smooth, we note that Theorem 1 follows from barrier type estimates and the boundary maximum principle for $p$ harmonic functions (see [2]). However constants then depend on a certain smoothness norm of $\phi$. We also remark that Theorem 1 is not new in $\mathbb{R}^{2}$. In fact in [5] it is shown that the conclusion of Theorem 1 is valid whenever $w$ lies on a quasicircle. Their proof however works only in two dimensions. Thus Theorem 1 is new for $p \neq 2,1<p<\infty, n>2$.

In the setting of starlike Lipschitz ring domains we are able to prove a refined version of Theorem 1 including the Hölder continuity of quotients of solutions. To formulate our results we have to introduce some more notation. A bounded domain $\Omega \subset \mathbb{R}^{n}$ is said to be starlike Lipschitz with respect to $\hat{x} \in \Omega$ provided

$$
\begin{aligned}
& \partial \Omega=\{\hat{x}+R(\omega) \omega: \omega \in \partial B(0,1)\} \\
& \quad \text { where } \log R: \partial B(0,1) \rightarrow \mathbb{R} \text { is Lipschitz on } \partial B(0,1) .
\end{aligned}
$$

We say that $D$ is a starlike Lipschitz ring domain with center $\hat{x}$ provided $D=\Omega \backslash \bar{\Omega}^{\prime}$ where $\Omega, \Omega^{\prime}$ are starlike Lipschitz domains with center $\hat{x}$ and $\bar{\Omega}^{\prime} \subset \Omega$. Let $R, R^{\prime}$ be the graph functions for $\partial \Omega, \partial \Omega^{\prime}$. We shall refer to $\left\|\log R \hat{\|}_{\partial B(0,1)}+\right\| \log R^{\prime} \hat{\|}_{\partial B(0,1)}$ as the Lipschitz constant for $D$. Observe that this constant is invariant under scaling about $\hat{x}$ and also that $\operatorname{diam} \Omega \approx d(\hat{x}, \partial \Omega)$, $\operatorname{diam} \Omega^{\prime} \approx d\left(\hat{x}, \partial \Omega^{\prime}\right)$, where $A \approx B$ means $A / B$ is bounded above and below by constants which depend only on $p, n$, and the Lipschitz constant for $D$. If $p$ is fixed, $1<p<\infty$, let $\hat{u}=\hat{u}(\cdot, p)$ be the $p$ capacitary function for $D$. That is $\hat{u} \equiv 1$ on $\partial \Omega^{\prime}, \hat{u} \equiv 0$ on $\partial \Omega$ in the sense of $W_{0}^{1, p}(\Omega)$ and $\hat{u}$ is $p$ harmonic in $D$. It is well known that $\hat{u}$ is unique and

$$
\int_{D}|\nabla \hat{u}|^{p} d x=\inf \left\{\int_{D}|\nabla \theta|^{p} d x\right\}
$$

where the infimum is taken over all $\theta \in C_{0}^{\infty}(\Omega)$ with $\theta \equiv 1$ on $\bar{\Omega}^{\prime}$. We are able to prove the following theorem on the boundary behaviour of $p$ capacitary functions. 
THEOREM 2. - Let $\hat{D}_{1}, \hat{D}_{2}$ be starlike Lipschitz ring domains with centers, $\hat{x}, \hat{y}$, respectively. For fixed $p, 1<p<\infty$, let $\hat{u}_{i}$ be the $p$ capacitary function for $\hat{D}_{i}$, and put $\tilde{u}_{i}=\min \left(\hat{u}_{i}, 1-\hat{u}_{i}\right)$ for $i=1,2$. Assume also that $w \in \partial \hat{D}_{1} \cap \partial \hat{D}_{2}, \hat{x}, \hat{y} \notin B(w, 16 r)$,

$$
B(w, 2 r) \cap \hat{D}_{1}=B(w, 2 r) \cap \hat{D}_{2},
$$

and $\bar{B}(w, 8 r)$ does not contain points in either both bounded components or both unbounded components of $\mathbb{R}^{n} \backslash \hat{D}_{i}$ for $i=1,2$. Then there exist $\alpha, c_{2}, 0<\alpha \leqslant 1 \leqslant c_{2}<\infty$, depending only on $p, n$, and the Lipschitz constants for $\hat{D}_{1}, \hat{D}_{2}$, such that if $w_{1}, w_{2}, \in \bar{B}(w, r) \cap \hat{D}_{1}$, then

$$
\left|\frac{\tilde{u}_{1}\left(w_{1}\right)}{\tilde{u}_{2}\left(w_{1}\right)}-\frac{\tilde{u}_{1}\left(w_{2}\right)}{\tilde{u}_{2}\left(w_{2}\right)}\right| \leqslant c_{2} \frac{\tilde{u}_{1}\left(a_{r}(w)\right)}{\tilde{u}_{2}\left(a_{r}(w)\right)}\left(\frac{\left|w_{1}-w_{2}\right|}{r}\right)^{\alpha},
$$

where $a_{r}(w)$ is a point in $\bar{B}(w, r) \cap \hat{D}_{1} \quad$ with $\quad d\left(a_{r}(w), \partial \hat{D}_{1}\right)=\sup \left\{d\left(y, \partial \hat{D}_{1}\right)\right.$ : $\left.y \in \bar{B}(w, r) \cap \hat{D}_{1}\right\}$.

Theorem 1 is proved at the end of Section 4. As noted earlier, the proof of this theorem uses Theorem 2 and a comparison argument involving $p$ capacitary functions. Thus we briefly outline the proof of Theorem 2 . We start by noting that if $\hat{u}(\cdot, \lambda), \lambda \in[0,1]$, is $p$ harmonic in a domain $G$, $\nabla \hat{u}(x, \lambda)$ is nonzero for $x \in G$, and if $\hat{u}$ is sufficiently smooth in $x, \lambda$, then $\zeta=\frac{\partial \hat{u}}{\partial \lambda}(\cdot, \lambda)$ satisfies, at $x$, the partial differential equation

$$
L \zeta=\nabla \cdot\left[(p-2)|\nabla \hat{u}|^{p-4}\langle\nabla \hat{u}, \nabla \zeta\rangle \nabla \hat{u}+|\nabla \hat{u}|^{p-2} \nabla \zeta\right]=0 .
$$

This follows from differentiating (1.2) for $\hat{u}$ with respect to $\lambda$. In (1.5) we have written $\nabla \hat{u}$ for $\nabla \hat{u}(\cdot, \lambda)$. Clearly,

$$
L \hat{u}(x, \cdot)=(p-1) \nabla \cdot\left[|\nabla \hat{u}|^{p-2} \nabla \hat{u}(x, \cdot)\right]=0 .
$$

(1.5) can be written in the form

$$
L \zeta=\sum_{i, j=1}^{n} \frac{\partial}{\partial x_{i}}\left[b_{i j}(x) \zeta_{x_{j}}(x)\right]=0,
$$

where at $x \in G$,

$$
b_{i j}(x)=|\nabla \hat{u}|^{p-4}\left[(p-2) \hat{u}_{x_{i}} \hat{u}_{x_{j}}+\delta_{i j}|\nabla \hat{u}|^{2}\right](x), \quad 1 \leqslant i, j \leqslant n,
$$

and $\delta_{i j}$ is the Kronecker $\delta$. Again we have written $\nabla \hat{u}$ for $\nabla \hat{u}(\cdot, \lambda)$. The first key observation in the proof of Theorem 2 is that $\hat{u}(\cdot, \lambda), \frac{\partial \hat{u}}{\partial \lambda}(\cdot, \lambda)$, both satisfy the divergence form partial differential equation (1.7).

To continue our outline of the proof of Theorem 2, the proof uses a delicate deformation technique for starlike Lipschitz ring domains. To describe this technique and to simplify matters, we consider the following special case of Theorem 2. Let $\hat{u}_{i}$ be the $p$ capacitary functions for starlike Lipschitz ring domains, $\hat{D}_{i}$, with $\hat{D}_{i}=\hat{\Omega}_{i} \backslash \bar{B}(\hat{x}, \rho), i=1,2, w \in \partial \hat{\Omega}_{1} \cap \partial \hat{\Omega}_{2}$, and

$$
d\left(\hat{x}, \partial \hat{\Omega}_{i}\right) / 4 \leqslant \rho \leqslant d\left(\hat{x}, \partial \hat{\Omega}_{i}\right) / 2 \text { for } i=1,2 .
$$

Let $\hat{R}_{i}, i=1,2$, be the corresponding graph functions for $\partial \hat{\Omega}_{i}$ and assume that $\hat{R}_{i}, i=1,2$, is infinitely differentiable on the manifold $\partial B(0,1)$. Put $\hat{R}(\tau)=\hat{R}_{2}^{\tau} \hat{R}_{1}^{1-\tau}, 0 \leqslant \tau \leqslant 1$, and 
let $\hat{\Omega}(\tau)$ be the starlike Lipschitz domain with center $\hat{x}$, graph function $\hat{R}(\tau)$, while $\hat{D}(\tau)=$ $\hat{\Omega}(\tau) \backslash \bar{B}(\hat{x}, \rho)$ is the corresponding ring domain. Let $\hat{u}(\cdot, \tau), \tau \in[0,1]$, be the $p$ capacitary function for $\hat{D}(\tau)$ so that $\hat{u}(\cdot, 0)=\hat{u}_{1}, \hat{u}(\cdot, 1)=\hat{u}_{2}$. In Lemma 2.5 we show that

$$
|\nabla \hat{u}(x, \tau)| \approx \frac{\hat{u}(x, \tau)}{d(x, \partial \hat{\Omega}(\tau))} \quad \text { whenever } x \in \hat{D}(\tau)
$$

This fact and Schauder type arguments imply (see Lemma 4.5) that $\hat{u}(x, \tau)$ is smooth in $x, \tau$ whenever $x \in \bigcup \hat{D}(\tau)$. Hence $\{\hat{u}(x, \tau)\}, \tau \in[0,1]$, is a smooth deformation of $\hat{u}_{1}(x)$ to $\hat{u}_{2}(x)$ and (1.5)-(1.8) hold with $\lambda$ replaced by $\tau$. Using this deduction we get

$$
\log \left(\frac{\hat{u}_{2}(x)}{\hat{u}_{1}(x)}\right)=\int_{0}^{1} \frac{\hat{u}_{\tau}(x, \tau)}{\hat{u}(x, \tau)} d \tau .
$$

It follows, from the assumptions in Theorem 2, that

$$
\hat{D}(\tau) \cap B(w, 2 r)=\hat{D}_{1} \cap B(w, 2 r) \quad \text { for all } \tau \in[0,1] .
$$

Furthermore it turns out, if for example $\hat{R}_{2} \geqslant \hat{R}_{1}$, that $\hat{u}_{\tau}>0$ in $\hat{D}(\tau)$ and $\hat{u}_{\tau}=0$ continuously on $B(w, 2 r) \cap \partial \hat{D}_{1}$. Therefore we see, in view of (1.10) and the first key observation, that in order to prove the above simplified version of Theorem 2 , it suffices to show that $\hat{u}_{\tau} / \hat{u}$ is Hölder continuous in $\hat{D}_{1} \cap B(w, r)$ with constants independent of $\tau, 0<\tau<1$. Thus the proof of Theorem 2 is, in this case, reduced to proving a boundary Harnack inequality for positive solutions to (1.5) vanishing on $B(w, 2 r) \cap \partial \hat{D}_{1}$. To prove such an inequality we note that if $p$ is near enough $2, p \neq 2$, then we can use (1.9) and argue as in [36] to deduce first that $|\nabla \hat{u}(\cdot, \tau)|^{p-2}$ extends to an $A_{2}$ weight on $\mathbb{R}^{n}$ and second apply results from [15-17] to get the desired boundary Harnack inequality. Thus in this case one first gets Hölder continuity of $\hat{u}_{\tau}(\cdot, \tau) / \hat{u}(\cdot, \tau)$ and then of $\hat{u}_{1} / \hat{u}_{2}$. In the general case, $1<p<\infty, p \neq 2$, we must work harder, as simple examples show that $h=|\nabla \hat{u}|^{p-2}(\cdot, \tau)$ need not be an $A_{2}$ weight. To get around this difficulty we use some Rellich type inequalities (see Lemmas 2.39, 2.45, 2.54) and a theorem of Kenig and Pipher [31] (see Theorem 3.11) to show directly in the spirit of Jerison and Kenig (see Lemma 3.13) that $\hat{u}_{\tau}(\cdot, \tau) / \hat{u}(\cdot, \tau)$ is Hölder continuous in $\hat{D}_{1} \cap B(w, r)$.

Finally we note that we currently cannot prove Hölder continuity in Theorem 1, using a similar variational type argument (as in Theorem 2), because we cannot prove a boundary Harnack inequality for the resulting partial differential equation satisfied by $\hat{u}_{\tau}, \hat{u}$. At the very least it appears that one needs to know that inequalities similar to (1.9) hold for $\tilde{u}, \tilde{v}$ in $B(w, r / c) \cap G$ for some large $c$ depending only on $p, n$, and the Lipschitz constant for $\phi$.

Next we formulate results on nontangential limits, at the boundary, for gradients of $p$ capacitary functions in starlike Lipschitz ring domains. In particular we state generalizations of work by Dahlberg [12] (Theorem 3), and results of Jerison and Kenig [25] (Theorem 4). To do this we shall need some more notation. Let $D=\Omega \backslash \bar{\Omega}^{\prime}$ be a starlike Lipschitz ring domain with center $\hat{x}$ and as previously, let $R, R^{\prime}$ be the graph functions for $\partial \Omega, \partial \Omega^{\prime}$. Let $w \in \partial D, r>0$, and suppose that

$$
\hat{x} \notin B(w, 8 r) \text {, as well as, either } B(w, 8 r) \cap \partial \Omega^{\prime}=\emptyset \text { or } B(w, 8 r) \cap \partial \Omega=\emptyset .
$$

We say that $B(w, 8 r) \cap \partial D$ is $C^{1}$ provided $\tilde{R}$ is continuously differentiable on $\{(y-\hat{x}) /|y-\hat{x}|$ : $y \in B(w, 8 r) \cap \partial D\}$, where $\tilde{R}=R^{\prime}$ if $B(w, 8 r) \cap \partial \Omega=\emptyset$ and $\tilde{R}=R$ when $B(w, 8 r) \cap \partial \Omega^{\prime}=\emptyset$. 
Given $b>1$ and $x \in \partial D \cap B(w, 2 r)$, let $\Gamma(x)=\{y \in D \cap B(w, 8 r):|y-x|<b d(y, \partial D)\}$. We note from elementary geometry that if $b$ is large enough (depending on the Lipschitz constant for $D$ ), then $\Gamma(x)$ contains the inside of a truncated cone with vertex $x$, axis parallel to $\hat{x}-x$, angle opening $\theta=\theta(b)>0$, and height $r$. Fix $b$ so that this property holds for all $x \in \partial D \cap B(w, 2 r)$. Given a measurable function $k$ on $D \cap B(w, 8 r)$ define the nontangential maximal function $N(k): \partial D \cap B(w, 2 r) \rightarrow \mathbb{R}$ of $k$ by

$$
N(k)(x)=\sup _{y \in \Gamma(x)}|k|(y) \quad \text { whenever } x \in \partial D \cap B(w, 2 r) .
$$

Let $H^{m}, 1 \leqslant m \leqslant n$, denote $m$-dimensional Hausdorff measure (see [40] for a definition) and let $L^{q}[\partial D \cap B(w, 2 r)], 1 \leqslant q \leqslant \infty$, be the usual space of $q$-th power $H^{n-1}$ integrable functions on $\partial D \cap B(w, 2 r)$. Given a measurable function $f$ on $\partial D \cap B(w, 2 r)$ we say that $f$ is of bounded mean oscillation on $\partial D \cap B(w, r)(f \in B M O(\partial D \cap B(w, r)))$ if for all $x \in \partial D \cap B(w, r)$ and $0<s \leqslant r$, there exists $0<A<\infty$ satisfying

$$
\int_{B(x, s) \cap \partial D}\left|f-f_{B}\right| d H^{n-1} \leqslant A s^{n-1} .
$$

Here $f_{B}$ denotes the average of $f$ on $B(x, s) \cap \partial D$ with respect to $H^{n-1}$ measure. The least such $A$ for which (1.14) holds will be denoted by $\|f\|$. We say that $f$ is in $\operatorname{VMO}(\partial D \cap B(w, r))$ provided for each $\epsilon>0$ there is a $\delta>0$ such that (1.14) holds with $A$ replaced by $\epsilon$ whenever $0<s<\min (\delta, r)$ and $x \in \partial D \cap B(w, r)$. Using this notation, Lemmas 2.39, 2.45, 2.54, and Theorem 3.11, we prove the following two theorems.

THEOREM 3. - Let D be a starlike Lipschitz ring domain with center $\hat{x}$ and $r, w$ as in (1.12). Let $u$ be the $p$ capacitary function for $D$. Then

$$
\lim _{y \in \Gamma(x), y \rightarrow x} \nabla u(y)=\nabla u(x) \quad \text { for } H^{n-1} \text { almost every } x \in \partial D .
$$

Furthermore, there exist $1 \leqslant c<\infty$ and $q, q>p$, depending only on $p, n$, and $\||\nabla \phi|\|_{\infty}$ such that

(a) $N(|\nabla u|) \in L^{q}(\partial D \cap B(w, 2 r))$,

(b)

$$
\int_{B(w, 2 r) \cap \partial D}|\nabla u|^{q} d H^{n-1} \leqslant c r^{(n-1)\left(\frac{p-1-q}{p-1}\right)}\left(\int_{B(w, 2 r) \cap \partial D}|\nabla u|^{p-1} d H^{n-1}\right)^{q /(p-1)},
$$

(c) $\log |\nabla u| \in B M O(\partial D \cap B(w, r))$ with $\| \log |\nabla u| \tilde{\|} \leqslant c$.

THEOREM 4. - If $\partial D \cap B(w, 8 r)$ is $C^{1}$ and $u, D, r, w$ are as in Theorem 3 , then

$$
\log |\nabla u| \in \operatorname{VMO}(\partial D \cap B(w, r)) .
$$

The rest of the paper is organized as follows. In Section 2 we state and derive some basic lemmas which will be used in the proof of Theorems 1-4. In Section 3 we introduce elliptic measure defined with respect to the partial differential equation in (1.7), (1.8), and derive a boundary Harnack inequality for positive solutions. In Section 4 we study variations of capacitary functions in smooth starlike Lipschitz ring domains and prove Theorem 1. In Section 5 we prove Theorems 2, 3, 4 .

$4^{\text {e }}$ SÉRIE - TOME $40-2007-\mathrm{N}^{\circ} 5$ 


\section{Basic estimates}

Let $\Omega^{+}, \Omega^{-}$be starlike Lipschitz domains with center $\hat{z}$ and $\bar{\Omega}^{-} \subset \Omega^{+}$. Let $R^{+}, R^{-}$be the graph functions for $\Omega^{+}, \Omega^{-}$and put $\hat{D}=\Omega^{+} \backslash \bar{\Omega}^{-}$. Let $\beta$ be the Lipschitz constant for $\hat{D}$. In the sequel, unless otherwise stated, $c$ will denote a positive constant $\geqslant 1$ (not necessarily the same at each occurrence), depending only on $p, n$, and $\beta$. In general, $c\left(a_{1}, \ldots, a_{n}\right)$ denotes a positive constant $\geqslant 1$, which depends on $p, n, \beta$ and $a_{1}, \ldots, a_{n}$, not necessarily the same at each occurrence. Let $\hat{u}$ be the $p$ capacitary function for $\hat{D}$ and put $\hat{u} \equiv 1$ on $\bar{\Omega}^{-}$while $\hat{u} \equiv 0$ on $\mathbb{R}^{n} \backslash \Omega^{+}$. With $\hat{u}$ now defined on $\mathbb{R}^{n}$ let $\max _{B(z, s)} \hat{u}, \min _{B(z, s)} \hat{u}$ be the essential supremum and infimum of $\hat{u}$ on $B(z, s)$ whenever $B(z, s) \subset \mathbb{R}^{n}$. We say that (1.12) holds for $w$, provided $w \in \partial \hat{D}$ and (1.12) is valid with $\hat{x}, \Omega, \Omega^{\prime}$ replaced by $\hat{z}, \Omega^{+}, \Omega^{-}$, respectively. To begin this section, we state some interior and boundary estimates for $\hat{u}$.

LEMMA 2.1. - Let $\hat{u}$ be the papacitary function for $\hat{D}$ and put $\tilde{u}=\min (\hat{u}, 1-\hat{u})$.

(a) If $B(w, 2 r) \subset \hat{D}$, or (1.12) holds for $w$, then

$$
r^{p-n} \int_{B(w, r / 2)}|\nabla \hat{u}|^{p} d x \leqslant c \max _{B(w, r)} \tilde{u}^{p} .
$$

(b) If $B(w, 2 r) \subset \hat{D}$, then $\max _{B(w, r)} \tilde{u} \leqslant c \min _{B(w, r)} \tilde{u}$.

(c) If $B(w, 2 r) \subset \hat{D}$, or (1.12) holds for $w$, then

$$
|\tilde{u}(x)-\tilde{u}(y)| \leqslant c\left(\frac{|x-y|}{r}\right)^{\alpha} \max _{B(w, 2 r)} \tilde{u} \quad \text { whenever } x, y \in B(w, r) .
$$

Proof. - (a) of Lemma 2.1 is a standard subsolution estimate. $(b)$ is a well-known Harnack inequality for positive solutions of $p$ Laplacian type. If $B(w, 2 r) \subset \hat{D}$, then $(c)$ is a well-known interior Hölder continuity estimate for solutions of $p$ Laplacian type (see [41] for these results). If (1.12) holds for $w$, then (c) follows from simple barrier type estimates (see also [19]).

LEMMA 2.2.-Let $\tilde{u}$ be as in Lemma 2.1 and suppose (1.12) holds for $w$. Then $\max _{B(w, 2 r)} \tilde{u} \leqslant c \tilde{u}\left(a_{r}(w)\right)$ where $a_{r}(w)$ is a point in $\bar{B}(w, r)$ with $d\left(a_{r}(w), \partial \hat{D}\right)=$ $\sup \{d(y, \partial \hat{D}): y \in \bar{B}(w, r) \cap \hat{D}\}$. Thus

$$
|\tilde{u}(x)-\tilde{u}(y)| \leqslant c\left(\frac{|x-y|}{r}\right)^{\alpha} \tilde{u}\left(a_{r}(w)\right) \quad \text { whenever } x, y \in B(w, r) .
$$

Proof. - The first inequality in Lemma 2.2 follows from a general argument using Lemma 2.1 often attributed to Carleson (see [10]). However Domar was apparently the first to use this argument (see [1]). The second inequality follows from the first inequality and Lemma $2.1(c)$.

LEMMA 2.3. $-\hat{u}$ has a representative in $W^{1, p}\left(\mathbb{R}^{n}\right)$ that has Hölder continuous partial derivatives in $\hat{D}$. That is, for some $\sigma \in(0,1]$ (depending only on $p, n)$ we have

$$
c^{-1}|\nabla \hat{u}(x)-\nabla \hat{u}(y)| \leqslant(|x-y| / r)^{\sigma} \max _{B(w, r)}|\nabla \hat{u}| \leqslant c r^{-1}(|x-y| / r)^{\sigma} \max _{B(w, 2 r)} \hat{u}
$$

whenever $x, y \in B(w, r / 2)$ and $B(w, 4 r) \subset \hat{D}$. 
Proof. - The proof of Lemma 2.3 can be found in [13], [36] or [43].

LEMMA 2.4. $-\hat{u}$ is $C^{\infty}$ in $\hat{D} \backslash\{x: \nabla \hat{u}(x)=0\}$. Moreover if $R^{+}, R^{-}$are infinitely differentiable $\left(R^{+}, R^{-} \in C^{\infty}[\partial B(0,1)]\right)$, then there exists an open neighborhood $N$ of $\partial \hat{D}$ such that $\min _{\hat{D} \cap N}|\nabla \hat{u}|>0$ and $\hat{u}$ has a $C^{\infty}$ extension to the closure of $\hat{D} \cap N$.

Proof. - If $R^{+}, R^{-}$are infinitely differentiable, then from Lemma 2.3 and a result of Lieberman [38] it follows that $\nabla \hat{u}$ has a Hölder $\gamma$ extension to the closure of $\hat{D}$ for some $\gamma \in(0,1]$, depending on $p, n$ and the $C^{2}$ norm for $\partial \hat{D}$. Using this result and barriers of the form

$$
x \rightarrow A|x-z|^{(p-n) /(p-1)}+B \quad \text { for } p \neq n, \quad A \log |x-z|+B \quad \text { for } p=n,
$$

where $z \in \hat{D}$ and $A, B$ are constants, we conclude that there exists a neighborhood $N$ of $\partial \hat{D}$ for which $\min _{\hat{D} \cap N}|\nabla \hat{u}|>0$. Second from (1.2) and this conclusion we see that $\hat{u}$ is a solution to a nondivergence form uniformly elliptic equation with Hölder continuous coefficients in the closure of $\hat{D} \cap N$. We now use Schauder theory (see [20, Chapters 6, 9]) and a bootstrap type argument to get that $\hat{u}$ has a $C^{\infty}$ extension to the closure of $\hat{D} \cap N$. A similar argument gives that $\hat{u}$ is infinitely differentiable in a neighborhood of each point $x \in \hat{D}$ where $\nabla \hat{u}(x) \neq 0$.

LEMMA 2.5. - Let ũ be as in Lemma 2.1 and suppose that (1.12) holds for w. There exists $c$ such that

$$
\begin{aligned}
& \text { (i) } 0<|\nabla \hat{u}(x)| \leqslant c\left\langle\frac{\hat{z}-x}{|\hat{z}-x|}, \nabla \hat{u}(x)\right\rangle \text { whenever } x \in \hat{D}, \\
& \text { (ii) } c^{-1} \tilde{u}(x) / d(x, \partial \hat{D}) \leqslant|\nabla \hat{u}(x)| \leqslant c \tilde{u}(x) / d(x, \partial \hat{D}) \quad \text { whenever } x \in \hat{D} \cap B(w, 3 r), \\
& \text { (iii) } \max _{B\left(x, \frac{s}{2}\right)} \sum_{i, j=1}^{n}\left|\hat{u}_{y_{i} y_{j}}\right| \leqslant c\left(s^{-n} \int_{B(x, 3 s / 4)} \sum_{i, j=1}^{n}\left|\hat{u}_{y_{i} y_{j}}\right|^{2} d y\right)^{1 / 2} \leqslant c^{2} \tilde{u}(x) / d(x, \partial \hat{D})^{2} \\
& \text { whenever } x \in \hat{D} \cap B(w, 2 r) \text { and } 0<s \leqslant d(x, \partial \hat{D}) .
\end{aligned}
$$

Proof. - Since (1.2) is invariant under translations we assume, as we may, that $\hat{z}=0$. We also temporarily assume that

$$
R^{+}, R^{-} \in C^{\infty}[\partial B(0,1)] .
$$

Let $\theta(x)=-\langle x, \nabla \hat{u}(x)\rangle, x \in \hat{D}$. Then from Lemma 2.4 we deduce that $\theta$ has a continuous extension to the closure of $\hat{D}$. We claim for some $\epsilon>0$ that

$$
\theta \geqslant \epsilon \quad \text { in } \hat{D} .
$$

To prove claim (2.7) first observe from (2.6) and Lemma 2.4 that there exists a neighborhood $N$ of $\partial D$ such that

$$
\theta \geqslant 2 \epsilon \text { in } \hat{D} \cap N
$$

for $\epsilon$ small enough. Second, given $\eta>0,0<\eta \leqslant 1 / 2$, let $v=v(\cdot, \eta)$ be the weak solution to

$$
\nabla \cdot\left[\left(\eta+|\nabla v|^{2}\right)^{(p / 2-1)} \nabla v\right]=0 \quad \text { in } \hat{D}
$$

with boundary values 1 on $\bar{\Omega}^{-}, 0$ on $\mathbb{R}^{n} \backslash \Omega^{+}$in the sense of the Sobolev space $W_{0}^{1, p}$. We note that Lemmas 2.1-2.4 are valid with $\hat{u}$ replaced by $v$. Moreover, an examination of the proofs in the references after these lemmas shows that the constants and Hölder exponents in

$4^{\text {e }}$ SÉRIE - TOME $40-2007-\mathrm{N}^{\circ} 5$ 
Lemmas 2.2, 2.3 can, for a fixed $p, 1<p<\infty$, be chosen independent of $\eta \in(0,1 / 2]$. Using this fact, we deduce that any $W^{1, p}$ weakly convergent subsequence of $\{v(\cdot, \eta)\}$ converges uniformly as $\eta \rightarrow 0$ to a weak solution, say $\tilde{v}$, to the $p$ Laplacian in $\hat{D}$ with continuous boundary values 1 on $\partial \Omega^{-}$and 0 on $\partial \Omega^{+}$. From the weak maximum principle for the $p$ Laplace equation, it follows that $\tilde{v}=\hat{u}$. Thus, $v(\cdot, \eta), \nabla v(\cdot, \eta) \rightarrow \hat{u}, \nabla \hat{u}$ as $\eta \rightarrow 0$, uniformly on compact subsets of $\hat{D}$. From Schauder type estimates it then follows for each positive integer $k$ that $\partial^{k} v \rightarrow \partial^{k} \hat{u}$ uniformly on compact subsets of $V=\hat{D} \backslash\{x: \nabla \hat{u}(x)=0\}$. Here $\partial^{k}$ denotes a $k$-th partial. Finally $v(\cdot, \eta)$ is infinitely differentiable in $\hat{D}$, as follows once again from Schauder theory and the fact that (2.9) is uniformly elliptic in $\hat{D}$.

Given $\xi \in \mathbb{R}^{n},|\xi|=1$, and $\eta \in(0,1 / 2]$ let $f=\langle\nabla v, \xi\rangle$. Differentiating (2.9) in the direction $\xi$ we get

$$
L^{*} f=\sum_{i, j=1}^{n} \frac{\partial}{\partial x_{i}}\left(b_{i j}^{*} f_{x_{j}}\right)=0
$$

in $\hat{D}$, where at $x \in \hat{D}$,

$$
b_{i j}^{*}=\left(\eta+|\nabla v|^{2}\right)^{(p / 2-2)}\left[(p-2) v_{x_{i}} v_{x_{j}}+\delta_{i j}\left(\eta+|\nabla v|^{2}\right)\right]
$$

for $1 \leqslant i, j \leqslant n$. In (2.11), $\delta_{i j}$ again denotes the Kronecker $\delta$. We have

$$
\begin{aligned}
& \min (p-1,1)\left(\eta+|\nabla v|^{2}\right)^{(p / 2-1)}|\xi|^{2} \\
& \leqslant \sum_{i, j=1}^{n} b_{i j}^{*} \xi_{i} \xi_{j} \leqslant \max (p-1,1)\left(\eta+|\nabla v|^{2}\right)^{(p / 2-1)}|\xi|^{2}
\end{aligned}
$$

at $x \in \hat{D}$. Let $\theta^{*}=\theta^{*}(\cdot, \eta)$ be defined on $\hat{D}$ by $\theta^{*}(x)=-\langle\nabla v(x), x\rangle, x \in \hat{D}$.

From (2.9), (2.10), we find at $x \in \hat{D}$, that

$$
L^{*}\left(\theta^{*}\right)=(p-2) \eta \nabla \cdot\left[\left(\eta+|\nabla v|^{2}\right)^{(p / 2-2)} \nabla v\right] .
$$

Set $\psi(x)=\min \left(\theta^{*}(x)-\epsilon, 0\right)$ and let $\zeta \in C_{0}^{\infty}(\hat{D})$ with $\zeta \equiv 1$ on $\hat{D} \backslash N$. Observe from (2.8) and uniform convergence of $\nabla v$ to $\nabla \hat{u}$, that for sufficiently small $\eta$, say $0<\eta \leqslant \eta_{0}$, we have $\psi \zeta^{2} \equiv 0$ in $\hat{D} \cap \bar{N}$. From this observation and the definition of $\zeta$ we see that $\nabla\left(\psi \zeta^{2}\right)=(\nabla \psi) \zeta^{2}$ for almost every $x$ in $\hat{D}$ (with respect to Lebesgue $n$ measure). Using this fact, (2.12), (2.13), and integrating by parts we get,

$$
\begin{aligned}
I & =\min (p-1,1) \int_{\hat{D}}\left(\eta+|\nabla v|^{2}\right)^{(p / 2-1)}|\nabla \psi|^{2} \zeta^{2} d x \leqslant \sum_{i, j=1}^{n} \int_{\hat{D}} b_{i j}^{*} \psi_{x_{i}} \theta_{x_{j}}^{*} \zeta^{2} d x \\
& =(p-2) \eta \int_{\hat{D}}\left(\eta+|\nabla v|^{2}\right)^{(p / 2-2)}\left\langle\nabla v, \nabla\left(\psi \zeta^{2}\right)\right\rangle d x \equiv J .
\end{aligned}
$$

From Cauchy's inequality, we have

$$
J \leqslant c I^{1 / 2} K^{1 / 2}
$$


where

$$
K=\eta^{2} \int_{\hat{D}}\left(\eta+|\nabla v|^{2}\right)^{(p / 2-3)}|\nabla v|^{2} \zeta^{2} d x .
$$

For small $\delta>0$ we write,

$$
K=\int_{\{|\nabla \hat{u}| \leqslant \delta\} \cap \hat{D}} \ldots+\int_{\{|\nabla \hat{u}|>\delta\} \cap \hat{D}} \ldots=K_{1}+K_{2} .
$$

Now $\lim _{\eta \rightarrow 0} K_{2}=0$, as we see from uniform convergence of $\nabla v$ to $\nabla \hat{u}$ on the support of $\zeta$. Also, $\lim \sup _{\eta \rightarrow 0} K_{1} \leqslant A \delta^{p}$ for some $1<A<\infty$, independent of $\delta$. Since $\delta>0$ is arbitrary, we conclude that $\lim _{\eta \rightarrow 0} K=0$. Using this equality, the Fatou lemma, and uniform convergence of $\partial^{k} v$ to $\partial^{k} \hat{u}$ on compact subsets of $V=\hat{D} \backslash\{x: \hat{u}(x)=0\}$, we deduce from (2.14), (2.15) that

$$
\int_{\{\theta<\epsilon\} \cap V}|\nabla \hat{u}|^{p-2}|\nabla \theta|^{2} \zeta^{2} d x=0
$$

which implies in view of (2.8) that either $\{\theta<\epsilon\}$ is empty or $\theta$ is constant on components of $\{\theta<\epsilon\} \cap V$. The latter possibility cannot occur. In fact from (2.8), the only possible boundary points of such components are points in $\hat{D} \backslash V$ where $\theta \equiv 0$. Thus $\theta \equiv 0$ in $\{\theta<\epsilon\} \cap D$. However this deduction is impossible, since from continuity of $\theta$, connectivity of $\hat{D}$, and (2.8) we would have to have $\theta=\epsilon / 2$ at some point in $\hat{D}$. Thus claim (2.7) is valid.

To continue the proof of Lemma 2.5, observe from assumption (2.6) and Lemma 2.4 that for $c$ large enough (depending only on $\beta$ ),

$$
g(x)=c \theta(x)-|x||\nabla \hat{u}(x)|>0
$$

for $x \in \partial \hat{D}$. Here we have also used the fact that $\nabla \hat{u}$ is normal to tangent planes through points of $\partial \hat{D}$ and starlike Lipschitzness of $\partial \hat{D}$. We assert that (2.16) also holds in $\hat{D}$, which clearly implies Lemma 2.5(i). To prove our assertion, let $L$ be the operator in (1.7), (1.8) defined relative to $\hat{u}$ (to get $L$ replace $v$ by $\hat{u}$ and put $\eta=0$ in the definition of $L^{*}$ in (2.10)). We note that $L \theta=0$ in $\hat{D}$, as follows from Lemma 2.4, (2.7), and either the discussion above (1.6) with $\hat{u}(\cdot, \lambda)$ replaced by $\hat{u}(\lambda x)$ or (2.13) with $\eta=0$. To show that $g$ cannot have a negative minimum in $\hat{D}$ it suffices to show

$$
L(|x||\nabla \hat{u}(x)|) \geqslant 0 \quad \text { for all } x \in \hat{D}
$$

as we find from (2.7) and a standard argument for uniformly elliptic PDE in divergence form. To this end observe from symmetry and smoothness of $\left\{b_{i j}(x)\right\}, 1 \leqslant i, j \leqslant n$, that at $x \in \hat{D}$,

$$
\begin{aligned}
L(|x||\nabla \hat{u}|)= & |\nabla \hat{u}| L(|x|)+|x| L(|\nabla \hat{u}|) \\
& +2|x|^{-1}|\nabla \hat{u}|^{-1}\left(\sum_{i, j, k=1}^{n} b_{i j} x_{i} \hat{u}_{x_{k}} \hat{u}_{x_{k} x_{j}}\right) .
\end{aligned}
$$

To simplify the calculations we note that solutions to the $p$ Laplacian (see (1.2)) remain solutions under rotations. Moreover, $g$ is invariant under rotations around the origin. Thus we assume, for fixed $x \in \hat{D}$, that

$$
\nabla \hat{u}=|\nabla \hat{u}| e_{n}
$$

$4^{\text {e }}$ SÉRIE - TOME $40-2007-\mathrm{N}^{\circ} 5$ 
since otherwise we change coordinate systems. From (1.8) or (2.11) with $\eta=0, v=\hat{u}$, and (2.19) we see at $x$ that

$$
\left\{\begin{array}{l}
b_{i j}=0 \text { for } i \neq j, \\
b_{i i}=|\nabla \hat{u}|^{p-2} \text { for } 1 \leqslant i<n, \\
b_{n n}=(p-1)|\nabla \hat{u}|^{p-2} .
\end{array}\right.
$$

Differentiating (1.8) and using (2.19) we find at $x$ that

$$
\left\{\begin{array}{l}
\frac{\partial b_{i j}}{\partial x_{i}}=0 \quad \text { for } i \neq j, 1 \leqslant i, j<n \\
\frac{\partial b_{i i}}{\partial x_{i}}=\frac{\partial b_{n i}}{\partial x_{n}}=(p-2)|\nabla \hat{u}|^{p-3} \hat{u}_{x_{i} x_{n}} \quad \text { when } i<n \\
\frac{\partial b_{i n}}{\partial x_{i}}=(p-2)|\nabla \hat{u}|^{p-3} \hat{u}_{x_{i} x_{i}} \quad \text { if } 1 \leqslant i<n \\
\frac{\partial b_{n n}}{\partial x_{n}}=(p-1)(p-2)|\nabla \hat{u}|^{p-3} \hat{u}_{x_{n} x_{n}} .
\end{array}\right.
$$

We have

$$
\begin{aligned}
|\nabla \hat{u}| L(|x|) & =|\nabla \hat{u}||x|^{-1} \sum_{i, j=1}^{n} \frac{\partial b_{i j}}{\partial x_{i}} x_{j}+|\nabla \hat{u}||x|^{-3} \sum_{i, j=1}^{n} b_{i j}\left(|x|^{2} \delta_{i j}-x_{i} x_{j}\right) \\
& =T_{1}+T_{2} .
\end{aligned}
$$

We note that (1.7) for $\hat{u},(2.20),(2.21)$ imply that

$$
(p-1) \hat{u}_{x_{n} x_{n}}+\sum_{i=1}^{n-1} \hat{u}_{x_{i} x_{i}}=0 .
$$

Using (2.21), (2.23) we get at $x$,

$$
\begin{aligned}
T_{1} & =(p-2)|\nabla \hat{u}|^{p-2}|x|^{-1}\left[\sum_{i=1}^{n-1}\left(2 x_{i} \hat{u}_{x_{i} x_{n}}+x_{n} \hat{u}_{x_{i} x_{i}}\right)+(p-1) x_{n} \hat{u}_{x_{n} x_{n}}\right] \\
& =2(p-2)|\nabla \hat{u}|^{p-2}|x|^{-1} \sum_{i=1}^{n-1} x_{i} \hat{u}_{x_{i} x_{n}} .
\end{aligned}
$$

Also, from (2.20) we deduce

$$
\begin{aligned}
T_{2} & =|\nabla \hat{u}|^{p-1}|x|^{-3}\left[\sum_{i=1}^{n-1}\left(|x|^{2}-x_{i}^{2}\right)+(p-1)\left(|x|^{2}-x_{n}^{2}\right)\right] \\
& =|\nabla \hat{u}|^{p-1}|x|^{-3}\left[(n+p-3)\left(\sum_{i=1}^{n-1} x_{i}^{2}\right)+(n-1) x_{n}^{2}\right] .
\end{aligned}
$$

Next we note that $\hat{u}_{x_{k}}$ is a classical solution to (1.7), (1.8), as we see from (2.7), the discussion above (1.5) with $\hat{u}(x, \lambda)=\hat{u}\left(x+\lambda e_{k}\right)$ and the chain rule (see also (2.10)). Using this observation, (2.19), and (2.20) we find at $x$ that 


$$
\begin{aligned}
|x| L(|\nabla \hat{u}|) & =|x| \sum_{i, j, k=1}^{n} \frac{\partial}{\partial x_{i}}\left[\left(b_{i j} \hat{u}_{x_{k} x_{j}}\right)\left(\hat{u}_{x_{k}}|\nabla \hat{u}|^{-1}\right)\right] \\
& =|x||\nabla \hat{u}|^{-1} \sum_{i, j=1}^{n} \sum_{k=1}^{n-1} b_{i j} \hat{u}_{x_{k} x_{i}} \hat{u}_{x_{k} x_{j}} \\
& =|x||\nabla \hat{u}|^{p-3}\left[\sum_{i, k=1}^{n-1} \hat{u}_{x_{k} x_{i}}^{2}+(p-1) \sum_{k=1}^{n-1} \hat{u}_{x_{k} x_{n}}^{2}\right] .
\end{aligned}
$$

Finally using (2.20) we see at $x$ that

$$
\begin{aligned}
& 2|x|^{-1}|\nabla \hat{u}|^{-1}\left(\sum_{i, j, k=1}^{n} b_{i j} x_{i} \hat{u}_{x_{k}} \hat{u}_{x_{k} x_{j}}\right) \\
& =2|x|^{-1}|\nabla \hat{u}|^{p-2}\left(\sum_{i=1}^{n-1} x_{i} \hat{u}_{x_{i} x_{n}}+(p-1) x_{n} \hat{u}_{x_{n} x_{n}}\right) .
\end{aligned}
$$

Using (2.22), (2.24)-(2.27) in (2.18) and gathering terms we obtain after some juggling that

$$
\begin{aligned}
|\nabla \hat{u}|^{3-p}|x|^{3} L(|x||\nabla \hat{u}|) & \\
= & {\left[(n+p-3)|\nabla \hat{u}|^{2}\left(\sum_{i=1}^{n-1} x_{i}^{2}\right)+2(p-1)|\nabla \hat{u}||x|^{2}\left(\sum_{i=1}^{n-1} x_{i} \hat{u}_{x_{i} x_{n}}\right)\right.} \\
& \left.+(p-1)|x|^{4} \sum_{i=1}^{n-1} \hat{u}_{x_{i} x_{n}}^{2}\right] \\
& +\left[(n-1)|\nabla \hat{u}|^{2} x_{n}^{2}+2(p-1)|\nabla \hat{u}||x|^{2} x_{n} \hat{u}_{x_{n} x_{n}}+|x|^{4} \sum_{i, k=1}^{n-1} \hat{u}_{x_{i} x_{k}}^{2}\right] .
\end{aligned}
$$

To complete the proof of (2.17) we show both terms in brackets in (2.28) are nonnegative. In fact from Schwarz's inequality we see that

$$
\left(\sum_{i=1}^{n-1} x_{i} \hat{u}_{x_{i} x_{n}}\right)^{2} \leqslant\left(\sum_{i=1}^{n-1} \hat{u}_{x_{i} x_{n}}^{2}\right) \cdot\left(\sum_{i=1}^{n-1} x_{i}^{2}\right) .
$$

Using (2.29) in the first term in brackets in (2.28) (along with $2 a b \leqslant a^{2}+b^{2}$ ) we deduce that this term is nonnegative. Also from (2.23) and Schwarz's inequality we find

$$
(p-1)^{2} \hat{u}_{x_{n} x_{n}}^{2} \leqslant(n-1) \sum_{i=1}^{n-1} \hat{u}_{x_{i} x_{i}}^{2} .
$$

Using (2.30) in the second term in brackets in (2.28) and Schwarz's inequality, we conclude that this term is also nonnegative. Thus (2.17) is true. From our earlier remarks we obtain (2.16) in $\hat{D}$. Hence Lemma 2.5(i) is true under assumption (2.6).

To continue the proof of Lemma 2.5 we note that the upper bound in Lemma 2.5(ii) follows from Lemma 2.3. We use (2.16) to prove the lower bound in Lemma 2.5(ii). We first show that

$$
\max _{B(x, s)} \theta \leqslant c \min _{B(x, s)} \theta \quad \text { whenever } B(x, 4 s) \subset \hat{D} .
$$

$4^{\text {e }}$ SÉRIE - TOME $40-2007-\mathrm{N}^{\circ} 5$ 
To prove (2.31) observe from (2.16) and (1.8) that there exists $c$ for which

$$
\begin{aligned}
c^{-2}|x|^{2-p} \theta(y)^{p-2}|\xi|^{2} & \leqslant c^{-1}|\nabla \hat{u}(y)|^{p-2}|\xi|^{2} \leqslant \sum_{i, j=1}^{n} b_{i j}(y) \xi_{i} \xi_{j} \\
& \leqslant c|\nabla \hat{u}(y)|^{p-2}|\xi|^{2} \leqslant c^{2}|x|^{2-p} \theta(y)^{p-2}|\xi|^{2}
\end{aligned}
$$

whenever $y \in B(x, 2 s)$ and $\xi \in \mathbb{R}^{n}$. Using (2.32) and (1.7) for $\theta$ we see that Moser iteration can be applied to powers of $\theta$ in the usual way (see [41]) in order to get (2.31). To prove the lower bound in Lemma 2.5(ii), we consider two cases. First suppose that $\partial \Omega^{-} \cap B(w, 8 r)=\emptyset$. From Lemmas 2.1, 2.2 we see that $\tilde{u} \approx \hat{u}$ in $B(w, 6 r) \cap \hat{D}$ with proportionality constants depending only on $p, n, \beta$. If $x \in B(w, 3 r) \cap \hat{D}$, we can draw a ray $l$ in $\hat{D}$ from $x$ to a point in $\bar{B}(x, d(x, \partial \hat{D})) \cap \partial \Omega^{+}$thanks to (1.12) for $w$. Let $y$ be the first point on $l$ (starting from $x$ ) with $\hat{u}(y)=\hat{u}(x) / 2$. Then from the mean value theorem of elementary calculus there exists $z$ on the part of $l$ between $x, y$ with

$$
\hat{u}(x) / 2=\hat{u}(x)-\hat{u}(y) \leqslant|\nabla \hat{u}(z)||y-x| .
$$

From Lemma 2.2 we deduce the existence of $c$ with

$$
y, z \in B\left[x,\left(1-c^{-1}\right) d(x, \partial \hat{D})\right] .
$$

From (2.34), (2.31), and (2.16) it follows that for some $c,|\nabla \hat{u}(z)| \leqslant c|\nabla \hat{u}(x)|$. Using this inequality in (2.33) we conclude that

$$
\hat{u}(x) \leqslant c|\nabla \hat{u}(x)| d(x, \partial \hat{D}) .
$$

Hence the lower bound in Lemma 2.5(ii) is valid if $w \in \partial \Omega^{+}$. A similar argument applies if $w \in \partial \Omega^{-}$. We omit the details. Thus Lemma $2.5(i i)$ is valid.

Finally Lemma 2.5(ii) implies that the PDE in (1.7), (1.8) is uniformly elliptic in $B(x, 3 d(x, \partial \hat{D}) / 4)$ with Hölder continuous coefficients involving derivatives of $\hat{u}$. Since derivatives of $\hat{u}$ satisfy (1.7) (as mentioned above (2.26)), we can differentiate (1.7) to get a divergence form PDE for second derivatives of $u$. DiGiorgi or Moser iteration can then be applied to get Lemma 2.5(iii). One can also obtain Lemma 2.5(iii) from Schauder type estimates for the nondivergence form PDE satisfied by $\hat{u}$ as in Lemma 2.4. Thus Lemma 2.5 is valid under assumption (2.6).

To complete the proof of Lemma 2.5 we show that assumption (2.6) is unnecessary. For this purpose let $R_{m}^{+}, R_{m}^{-} \in C^{\infty}(\partial B(0,1))$ for $m=1,2, \ldots$, with $\| \log R_{m}^{*} \hat{\|} \leqslant k \beta$, and $R_{m}^{*} \rightarrow R^{*}$ as $m \rightarrow \infty$ uniformly on $\partial B(0,1)$ whenever $* \in\{+,-\}$. Here $k$ depends only on $n$. Let $\hat{D}_{m}, \hat{u}_{m}$ be the ring domain and $p$ capacitary function with center $\hat{z}$, corresponding to $R_{m}^{+}, R_{m}^{-}$. From Lemmas 2.2, 2.3 we see that $\hat{u}_{m}, \nabla \hat{u}_{m}$ converge uniformly on compact subsets of $\hat{D}$ to $\hat{u}, \nabla \hat{u}$. We apply Lemma 2.5 to each $\hat{u}_{m}$. Since the constants in this lemma and (2.31) are independent of $m$ we conclude that Lemma 2.5 also holds for $\hat{u}$ without hypothesis (2.6). The proof of Lemma 2.5 is now complete.

Lemma 2.35. - Let $\tilde{u}, \hat{u}, \hat{D}$, be as in Lemma 2.5. There exists unique finite positive Borel measures $\mu^{+}, \mu^{-}$on $\mathbb{R}^{n}$ with support in $\partial \Omega^{+}, \partial \Omega^{-}$, respectively, such that if $\mu=\mu^{+}-\mu^{-}$, then

(a) $\int|\nabla \hat{u}|^{p-2}\langle\nabla \hat{u}, \nabla \phi\rangle d x=-\int \phi d \mu$ whenever $\phi \in C_{0}^{\infty}\left(\mathbb{R}^{n}\right)$.

(b) If $w$ satisfies (1.12) then there exists $c$ such that $c^{-1} r^{p-n} \tilde{\mu}[B(w, 4 r)] \leqslant \tilde{u}\left(a_{r}(w)\right)^{p-1} \leqslant$ $c r^{p-n} \tilde{\mu}[B(w, r / 2)]$, where $\tilde{\mu}=\mu^{+}$if $B(w, 8 r) \cap \partial \Omega^{-}=\emptyset$ and $\tilde{\mu}=\mu^{-}$if $B(w, 8 r) \cap$ $\partial \Omega^{+}=\emptyset$. 
Proof. - Existence and uniqueness of $\mu$ satisfying Lemma 2.35(a) is easily proved using Lemmas 2.1 and 2.2 (see [5] or [21] for a proof). Assuming Lemma 2.35(a) one gets the lefthand inequality in Lemma $2.35(b)$ by first choosing $\phi \in C_{0}^{\infty}(B(w, 6 r))$ with $\phi \equiv 1$ on $B(w, 4 r)$ and then using Lemma 2.1. The right-hand inequality in Lemma $2.35(b)$ is essentially proved in [32, Lemma 3.1] (see also [14, Lemma 1]).

From Lemma 2.5 it is easily seen that

$$
\begin{aligned}
D(t)= & \{x: t<\hat{u}(x)<1\} \text { and } D^{\prime}(t)=\{x: 0<\hat{u}(x)<t\}, 0<t<1, \\
& \text { are starlike Lipschitz ring domains with center } \hat{z} \\
& \text { and constants depending only on } \beta .
\end{aligned}
$$

Moreover, $(1-t)^{-1} \max \{\hat{u}-t, 0\}$ and $\min \{\hat{u} / t, 1\}$ are the $p$ capacitary functions for $D(t), D^{\prime}(t)$, respectively. If $B(w, 8 r) \cap \Omega^{-}=\emptyset$, let $\mu_{t}, \mu^{-}$, be the measures corresponding to $\max \{\hat{u}-t, 0\}$ in $D(t)$, as in Lemma 2.35. We note that

$$
d \mu_{t}(w)=|\nabla \hat{u}|^{p-1}(w) d H^{n-1}(w) \quad \text { for } w \in\{x: \hat{u}(x)=t\}
$$

as follows from Lemma $2.35(a)$ with $\mu^{+}$replaced by $\mu_{t}$, Lemmas $2.4,2.5$, and integration by parts. A similar argument gives that $\mu_{t}, \mu^{+}$are the measures corresponding to $\min (u, t)$ in $D^{\prime}(t)$. Moreover, since $\hat{u} \in W^{1, p}\left(\mathbb{R}^{n}\right)$, it is easily deduced from Lemma $2.35(a)$ that

$$
\mu_{t} \rightarrow \mu^{+}, \mu^{-} \text {weakly in the sense of measures as } t \rightarrow 0,1 \text { respectively. }
$$

Next we have the following reverse Hölder inequality.

LEMMA 2.39. - Let $\mu, w$ be as in Lemma 2.35. Then $d \mu / d H^{n-1}= \pm k^{p-1}$ on $B(w, 8 r) \cap \partial \hat{D}$ where $k \geqslant 0$ with

$$
\int_{B(w, 2 r) \cap \partial \hat{D}} k^{p} d H^{n-1} \leqslant c r^{-\frac{n-1}{p-1}}\left(\int_{B(w, 2 r) \cap \partial \hat{D}} k^{p-1} d H^{n-1}\right)^{p /(p-1)} .
$$

Proof. - Again we consider two cases. If $B(w, 8 r) \cap \Omega^{-}=\emptyset$, we first show that Lemma 2.39 is valid with $\mu^{+}$replaced by $\mu_{t}$ for $t$ sufficiently near 0 . Again we assume that $\hat{z}=0$. For $t$ near 0 , and $2 r<s<3 r$, we note from Lemmas $2.4,2.5$ that integration by parts can be used to get

$$
\begin{aligned}
\int_{\partial[B(w, s) \cap D(t)]}\langle x, \nu\rangle|\nabla \hat{u}|^{p} d H^{n-1}= & \int_{B(w, s) \cap D(t)} \nabla \cdot\left(x|\nabla \hat{u}|^{p}\right) d x \\
= & n \int_{B(w, s) \cap D(t)}|\nabla \hat{u}|^{p} d x \\
& +\sum_{i, j=1}^{n} \int_{B(w, s) \cap D(t)} p|\nabla \hat{u}|^{p-2} x_{j} \hat{u}_{x_{i}} \hat{u}_{x_{i} x_{j}} d x
\end{aligned}
$$

$4^{\mathrm{e}}$ SÉRIE - TOME $40-2007-\mathrm{N}^{\circ} 5$ 
where $\nu$ denotes the outer unit normal to the boundary of $B(w, s) \cap D(t)$. Now from (1.2) and integration by parts, we deduce

$$
\begin{aligned}
& \sum_{i, j=1}^{n} \int_{B(w, s) \cap D(t)} p|\nabla \hat{u}|^{p-2} x_{j} \hat{u}_{x_{i}} \hat{u}_{x_{i} x_{j}} d x \\
& =-p \int_{B(w, s) \cap D(t)}|\nabla \hat{u}|^{p} d x+p \int_{\partial[B(w, s) \cap D(t)]}\langle x, \nabla \hat{u}\rangle\langle\nabla \hat{u}, \nu\rangle|\nabla \hat{u}|^{p-2} d H^{n-1} .
\end{aligned}
$$

Using this equality in (2.40) we get

$$
\begin{aligned}
I & =\int_{\partial[B(w, s) \cap D(t)]}\langle x, \nu\rangle|\nabla \hat{u}|^{p} d H^{n-1} \\
& -p \int_{\partial[B(w, s) \cap D(t)]}\langle x, \nabla \hat{u}\rangle\langle\nabla \hat{u}, \nu\rangle|\nabla \hat{u}|^{p-2} d H^{n-1} \\
& =(n-p) \int_{B(w, s) \cap D(t)}|\nabla \hat{u}|^{p} d x .
\end{aligned}
$$

We note that $\nu=-\frac{\nabla \hat{u}}{|\nabla \hat{u}|}$ on $\partial D(t) \cap B(w, s)$. Thus from (2.41)

$$
(p-1) \int_{\partial D(t) \cap B(w, s)}\langle x, \nabla \hat{u}\rangle|\nabla \hat{u}|^{p-1} d H^{n-1}=I+E
$$

where

$$
|E| \leqslant(p+1) \int_{\partial B(w, s) \cap D(t)}|x||\nabla \hat{u}|^{p} d H^{n-1} .
$$

Next choose $s \in(2 r, 3 r)$ so that

$$
\int_{\partial B(w, s) \cap D(t)}|\nabla \hat{u}|^{p} d H^{n-1} \leqslant 2 r^{-1} \int_{B(w, 3 r) \cap D(t)}|\nabla \hat{u}|^{p} d x .
$$

This choice is possible from weak type estimates. Using these inequalities in $(2.42), d(0, \partial \hat{D}) \approx$ $\operatorname{diam} \hat{D}$, and $(2.5)(i)$, we deduce

$$
\int_{B(w, s) \cap \partial D(t)}|x||\nabla \hat{u}|^{p} d H^{n-1} \leqslant c[d(0, \partial \hat{D}) / r] \int_{B(w, 3 r) \cap D(t)}|\nabla \hat{u}|^{p} d x+|I| .
$$


Then from (2.43), (2.41), (2.37), Lemmas 2.1, 2.2, 2.35 with $\hat{D}, \hat{u}$, replaced by $D(t)$, $(1-t)^{-1} \max (\hat{u}-t, 0)$, we find that

$$
\begin{aligned}
& c^{-2} \int_{B(w, s) \cap \partial D(t)}|\nabla \hat{u}|^{p} d H^{n-1} \leqslant c^{-1} r^{-1} \int_{B(w, 3 r) \cap D(t)}|\nabla \hat{u}|^{p} d x \\
& \leqslant r^{-\frac{n-1}{p-1}}\left(\int_{B(w, 2 r) \cap \partial D(t)}|\nabla \hat{u}|^{p-1} d H^{n-1}\right)^{p /(p-1)} \leqslant c r^{-p+n-1} \hat{u}\left(a_{3 r}(w)\right)^{p} \\
& =c r^{-\frac{n-1}{p-1}}\left(\mu_{t}[B(w, 2 r)]\right)^{p /(p-1)} .
\end{aligned}
$$

In (2.44) we have also used the fact that $d(0, \partial \hat{D})>r$. Thus Lemma 2.39 holds with $k=|\nabla \hat{u}|$ and $\mu^{+}, \hat{D}$ replaced by $\mu_{t}, D(t)$. Using the change of variables formula we can pull back each $\mu_{t}$ to a measure on $\partial B(0,1)$. In view of (2.44) and (2.36) we see that the Radon-Nikodym derivative of each pullback measure with respect to $H^{n-1}$ measure on $\partial B(0,1)$, satisfies a $L^{p /(p-1)}$ reverse Hölder inequality on $\{x /|x|: x \in B(w, 2 r) \cap \partial D(t)\}$. Moreover $L^{p /(p-1)}$ and Hölder constants depend only on $p, n, \beta$, for small $t>0$. Hence any sequence of these derivatives has a subsequence that converges weakly in $L^{p /(p-1)}$. Using these observations, lower semicontinuity of the norm in $L^{p /(p-1)}$ under weak convergence, (2.38), and letting $t \rightarrow 0$ we get the RadonNikodym derivative of the pullback of $\mu^{+}$on $B(w, 2 r) \cap \partial \hat{D}$ satisfies a $L^{p /(p-1)}$ reverse Hölder inequality. Transforming back, we conclude that Lemma 2.39 is true when $B(w, 8 r) \cap \partial \Omega^{-}=\emptyset$. If $B(w, 8 r) \cap \partial \Omega^{+}=\emptyset$, we can use the same argument with $D(t)$ replaced by $D^{\prime}(t)$. Letting $t \rightarrow 1$ and taking limits as above we get Lemma 2.39 in this case also.

We use Lemma 2.39 to prove the following localization lemma.

LEMmA 2.45. - Let $\tilde{u}, \hat{u}$, and $\hat{D}$ be as in Lemma 2.5. Let $w$ satisfy (1.12) and let $w^{\prime} \in \hat{D}$ be the point on the ray from $\hat{z}$ through $w$ with $\left|w-w^{\prime}\right|=r / 4$. There exist $c$ and a starlike Lipschitz domain $\tilde{\Omega} \subset B(w, 2 r) \cap \hat{D}$ with center at $w^{\prime}$,

$$
c H^{n-1}[\partial \tilde{\Omega} \cap B(w, r) \cap \partial \hat{D}] \geqslant r^{n-1},
$$

and Lipschitz constant $\beta^{\prime} \leqslant c(\beta+1)$. Moreover if $x \in \tilde{\Omega}$, then

$$
c^{-1} r^{-1} \tilde{u}\left(w^{\prime}\right) \leqslant|\nabla \hat{u}(x)| \leqslant c r^{-1} \tilde{u}\left(w^{\prime}\right) .
$$

Proof. - As usual we consider two cases. First suppose that $B(w, 8 r) \cap \partial \Omega^{-}=\emptyset$. From Lipschitzness of $\partial \hat{D}$ and basic geometry we deduce the existence of $c^{\prime}$ (depending only on $p, n, \beta)$ such that if

$$
\begin{aligned}
& \hat{R}(\omega)=\left|x-w^{\prime}\right| \quad \text { when } \omega=\frac{x-w^{\prime}}{\left|x-w^{\prime}\right|}, x \in B\left(w, r / c^{\prime}\right) \cap \partial \hat{D}, \\
& \text { then } \log \hat{R} \text { is Lipschitz on } E=\left\{\frac{x-w^{\prime}}{\left|x-w^{\prime}\right|}: x \in B\left(w, r / c^{\prime}\right) \cap \partial \hat{D}\right\} \\
& \text { with } \| \log \hat{R} \hat{\|}_{E} \leqslant c^{\prime}(\beta+1) .
\end{aligned}
$$

One proof of (2.46) is to observe that it is equivalent to the statement that the cones $\Gamma(x), x \in$ $B\left(w, r / c^{\prime}\right) \cap \partial \hat{D}$, defined above (1.13), each contain $w^{\prime}$. (2.46) can also be deduced from 
basic trigonometry using starlike Lipschitzness of $\hat{D}$ with respect to $\hat{z}$. Let $r^{\prime}=r / c^{\prime}$ and for $x \in B\left(w, r^{\prime}\right) \cap \partial \hat{D}$ set

$$
\begin{aligned}
& M_{1}(x)=\inf _{0<s<r^{\prime}} s^{1-n} \int_{B(x, s) \cap \partial \hat{D}} k^{p-1} d H^{n-1}, \\
& M_{2}(x)=\sup _{0<s<r^{\prime}} s^{1-n} \int_{B(x, s) \cap \partial \hat{D}} k^{p-1} d H^{n-1} .
\end{aligned}
$$

We claim there exist a compact set $F \subset B\left(w, r^{\prime} / 4\right) \cap \partial \hat{D}$ and $c^{\prime \prime}$ (depending only on $p, n, \beta$ ) with

$$
c^{\prime \prime} M_{1}>\left(r^{\prime}\right)^{1-p} \hat{u}\left(a_{r^{\prime}}(w)\right)^{p-1} \quad \text { on } F \quad \text { and } \quad c^{\prime \prime} H^{n-1}(F)>\left(r^{\prime}\right)^{n-1} .
$$

In fact if

$$
\begin{gathered}
\epsilon=\left(1 / c^{\prime \prime}\right)\left(r^{\prime}\right)^{1-p} \hat{u}\left(a_{r^{\prime}}(w)\right)^{p-1}, \\
G=\left\{x \in B\left(w, r^{\prime} / 4\right) \cap \partial \hat{D}: M_{1}(x) \leqslant \epsilon\right\},
\end{gathered}
$$

then by a standard covering argument there exists $\left\{B\left(x_{i}, r_{i}\right)\right\}$ with $x_{i} \in G, 0<r_{i} \leqslant r^{\prime}$, $G \subset \bigcup_{i} B\left(x_{i}, r_{i}\right)$ and $\left\{B\left(x_{i}, r_{i} / 10\right)\right\}$ pairwise disjoint. Also,

$$
\int_{B\left(x_{i}, r_{i}\right) \cap \partial \hat{D}} k^{p-1} d H^{n-1} \leqslant 2 \epsilon r_{i}^{n-1} \text { for each } i .
$$

Using these facts and $H^{n-1}\left[B\left(x_{i}, r_{i} / 10\right) \cap \partial \hat{D}\right] \approx r_{i}^{n-1}$, we get

$$
\begin{aligned}
\int_{G} k^{p-1} d H^{n-1} & \leqslant \sum_{i} \int_{B\left(x_{i}, r_{i}\right) \cap \partial \hat{D}} k^{p-1} d H^{n-1} \\
& \leqslant 2 \epsilon \sum_{i} r_{i}^{n-1} \leqslant c \epsilon\left(r^{\prime}\right)^{n-1} .
\end{aligned}
$$

On the other hand if $\Lambda=B\left(w, r^{\prime} / 4\right) \cap \partial \hat{D} \backslash G$, then from Lemmas 2.39, 2.35, with $r$ replaced by $r^{\prime} / 8$, and Hölder's inequality, we get for some $c$,

$$
\begin{aligned}
\int_{\Lambda} k^{p-1} d H^{n-1} & \leqslant H^{n-1}(\Lambda)^{1 / p} \cdot\left(\int_{B\left(w, r^{\prime} / 4\right) \cap \partial \hat{D}} k^{p} d H^{n-1}\right)^{1-1 / p} \\
& \leqslant c\left[\left(r^{\prime}\right)^{1-n} H^{n-1}(\Lambda)\right]^{1 / p} \cdot \int_{B\left(w, r^{\prime} / 4\right) \cap \partial \hat{D}} k^{p-1} d H^{n-1} \\
& \leqslant c^{2}\left[\left(r^{\prime}\right)^{1-n} H^{n-1}(\Lambda)\right]^{1 / p}\left(r^{\prime}\right)^{n-p} \hat{u}\left(a_{r^{\prime}}(w)\right)^{p-1}
\end{aligned}
$$

Since

$$
\left(r^{\prime}\right)^{n-p} \hat{u}\left(a_{r^{\prime}}(w)\right)^{p-1} \leqslant c \int_{B\left(w, r^{\prime} / 4\right) \cap \partial \hat{D}} k^{p-1} d H^{n-1}
$$


we can add (2.48), (2.49) to get after division by $\left(r^{\prime}\right)^{n-p} \hat{u}\left(a_{r^{\prime}}(w)\right)^{p-1}$ that for some $c$,

$$
c^{-1} \leqslant\left[\left(r^{\prime}\right)^{1-n} H^{n-1}(\Lambda)\right]^{1 / p}+1 / c^{\prime \prime} .
$$

Clearly (2.50) implies (2.47) with $F$ replaced by $\Lambda$. A standard measure theory argument then shows that we can replace $\Lambda$ by suitable $F$ compact, $F \subset \Lambda$. Thus (2.47) is valid.

Fix $c^{\prime \prime}$ so that (2.47) holds. To continue, we use the Hardy-Littlewood Maximal theorem (see [42, ch. 1]) and Lemmas 2.35, 2.39, to find $F^{\prime}$ compact, $F^{\prime} \subset F$ and $\bar{c} \geqslant c^{\prime \prime}$ such that

$$
M_{2} \leqslant \bar{c}\left(r^{\prime}\right)^{1-p} \hat{u}\left(a_{r^{\prime}}(w)\right)^{p-1} \text { on } F^{\prime} \quad \text { and } \quad \bar{c} H^{n-1}\left(F^{\prime}\right) \geqslant\left(r^{\prime}\right)^{n-1} .
$$

Combining this inequality with (2.47) and using Harnack's inequality (Lemma 2.1(b)), we have for some $c$,

$$
\begin{aligned}
& c^{-1} r^{1-p} \hat{u}\left(w^{\prime}\right)^{p-1} \leqslant s^{1-n} \int_{B(x, s) \cap \partial \hat{D}} k^{p-1} d H^{n-1} \leqslant c r^{1-p} \hat{u}\left(w^{\prime}\right)^{p-1} \\
& \text { for } x \in F^{\prime} \text { and } 0<s<r^{\prime} \text { where } H^{n-1}\left(F^{\prime}\right) \geqslant c^{-1} r^{n-1} .
\end{aligned}
$$

Next we draw all rays from points in $\bar{B}\left(w^{\prime}, r / c\right)$ to points in $F^{\prime}$. Let $\tilde{\Omega}$ be the interior of this set. Using (2.46) it is easily seen for $c$ large enough that $\tilde{\Omega}$ is starlike Lipschitz with center at $w^{\prime}$ and Lipschitz constant $\leqslant c(\beta+1)$. Also from (2.51) we see that

$$
c H^{n-1}[\partial \tilde{\Omega} \cap B(w, r) \cap \partial \hat{D}] \geqslant r^{n-1} .
$$

If $x \in \tilde{\Omega}$ and $d(x, \partial \hat{D}) \geqslant r^{\prime} / 10^{5}$, then from Lemma 2.5(ii) and Harnack's inequality, we find that

$$
c^{-1} r^{-1} \hat{u}\left(w^{\prime}\right) \leqslant|\nabla \hat{u}(x)| \leqslant c r^{-1} \hat{u}\left(w^{\prime}\right) .
$$

Otherwise there exists $\tilde{x} \in F^{\prime}$ with $|x-\tilde{x}| \leqslant c d(x, \partial \hat{D})$. Choose $s, 0<s<r^{\prime}$, such that $s \leqslant|x-\tilde{x}| \leqslant c s$. Using Lemmas 2.35, 2.39, 2.5(ii), (2.51), and Harnack's inequality we conclude that

$$
\begin{aligned}
|\nabla \hat{u}(x)|^{p-1} & \approx[\hat{u}(x) / d(x, \partial \hat{D})]^{p-1} \approx s^{1-n} \int_{B(\tilde{x}, s) \cap \partial \hat{D}} k^{p-1} d H^{n-1} \\
& \approx r^{1-p} \hat{u}\left(w^{\prime}\right)^{p-1}
\end{aligned}
$$

where all proportionality constants depend only on $p, n, \beta$. Thus (2.52) holds in this case also. The proof of Lemma 2.45 is now complete when $B(w, 8 r) \cap \partial \Omega^{-}=\emptyset$. An essentially identical argument holds if $B(w, 8 r) \cap \partial \Omega^{+}=\emptyset$.

Finally in this section we use Lemmas $2.45,2.5$ to show that if

$$
d \tilde{\sigma}(y)=d(y, \partial \tilde{\Omega}) \max _{B\left(y, \frac{1}{2} d(y, \partial \tilde{\Omega})\right)}\left\{|\nabla \hat{u}|^{2 p-6} \sum_{i, j=1}^{n} \hat{u}_{x_{i} x_{j}}^{2}\right\} d y
$$

when $y \in \tilde{\Omega}$, then $\tilde{\sigma}$ is a Carleson measure on $\tilde{\Omega}$. More specifically we prove the following lemma.

$4^{\mathrm{e}}$ SÉRIE - TOME $40-2007-\mathrm{N}^{\circ} 5$ 
LEMMA 2.54. - Let $w, w^{\prime}, r, \tilde{\Omega}$ be as in Lemma 2.45. If $z \in \partial \tilde{\Omega}$ and $0<s<r$, then

$$
\tilde{\sigma}(B(z, s) \cap \tilde{\Omega}) \leqslant c s^{n-1}\left(\tilde{u}\left(w^{\prime}\right) / r\right)^{2 p-4} .
$$

Proof. - We only give the proof when $B(w, 8 r) \cap \Omega^{-}=\emptyset$. Let $\tilde{R}$ be the graph function for $\tilde{\Omega}$. We let for, $0<\epsilon<\frac{1}{100} \min _{\partial B(0,1)} \tilde{R}, R=\tilde{R}-\epsilon$ and let $\Omega$ be the starlike Lipschitz domain with center $w^{\prime}$, graph function $R$. If $z \in \partial \tilde{\Omega}$ and $0<s \leqslant \frac{1}{2} d(z, \partial \hat{D})$, we can use Lemmas 2.45, 2.5, and the fact that $d(y, \partial \tilde{\Omega}) \leqslant d(y, \partial \hat{D})$ for $y \in \tilde{\Omega}$, to get that Lemma 2.54 is valid in this case. Otherwise, we use a simplified version of the argument in [34,35]. Again, let $L,\left(b_{i j}\right)$, $1 \leqslant i, j \leqslant n$, be as in (1.7), (1.8), defined relative to $\hat{u}, \hat{D}$. Since derivatives of $\hat{u}$ are solutions to $L \zeta=0$ (see the remark above (2.26)), we find at $x \in \hat{D}$,

$$
\begin{aligned}
L\left(|\nabla \hat{u}|^{2}\right) & =\sum_{i, j=1}^{n} \frac{\partial}{\partial x_{i}}\left[b_{i j} \frac{\partial}{\partial x_{j}}\left(|\nabla \hat{u}|^{2}\right)\right] \\
& =2 \sum_{i, j=1}^{n} b_{i j} \hat{u}_{x_{i} x_{j}} \hat{u}_{x_{i} x_{j}} \geqslant \min (1, p-1)|\nabla \hat{u}|^{p-2} \sum_{i, j=1}^{n} \hat{u}_{x_{i} x_{j}}^{2} .
\end{aligned}
$$

Also we note from (2.5)(ii), (iii) and Lemma 2.45 that

$$
\begin{aligned}
& \int_{B(z, s) \cap \Omega} d(y, \partial \Omega) \max _{B\left(y, \frac{1}{2} d(y, \partial \Omega)\right)}\left\{|\nabla \hat{u}|^{2 p-6} \sum_{i, j=1}^{n} \hat{u}_{x_{i} x_{j}}^{2}\right\} d y \\
& \leqslant c \int_{B(z, s) \cap \Omega} d(y, \partial \Omega)^{1-n}\left(\int_{B\left(y, \frac{3}{4} d(y, \partial \Omega)\right)}|\nabla \hat{u}|^{2 p-6} \sum_{i, j=1}^{n} \hat{u}_{x_{i} x_{j}}^{2} d x\right) d y \\
& \leqslant c \int_{B(z, 4 s) \cap \Omega} d(y, \partial \Omega)|\nabla \hat{u}(y)|^{2 p-6} \sum_{i, j=1}^{n} \hat{u}_{y_{i} y_{j}}^{2} d y
\end{aligned}
$$

where the third integral is obtained from the second integral by interchanging the order of integration. Define $\sigma$ as above Lemma 2.54 with $\tilde{\Omega}$ replaced by $\Omega$. Using (2.55), (2.56) and once again Lemmas $2.5,2.45$ we see that

$$
\begin{aligned}
\sigma[B(z, s) \cap \Omega] & \leqslant c \int_{B(z, 4 s) \cap \Omega} d(y, \partial \Omega)|\nabla \hat{u}(y)|^{2 p-6} \sum_{i, j=1}^{n} \hat{u}_{y_{i} y_{j}}^{2} d y \\
& \leqslant c\left(\frac{\hat{u}\left(w^{\prime}\right)}{r}\right)^{(p-5)} \int_{B(z, 4 s) \cap \Omega} \hat{u}(y) L\left(|\nabla \hat{u}|^{2}\right)(y) d y .
\end{aligned}
$$

We note that the divergence theorem can be used in $B(z, 4 s) \cap \Omega$. Since $L \hat{u}=0$ in $\hat{D}$ we have

$$
\int_{B(z, 4 s) \cap \Omega} \hat{u} L\left(|\nabla \hat{u}|^{2}\right) d y=\int_{B(z, 4 s) \cap \Omega}\left[\hat{u} L\left(|\nabla \hat{u}|^{2}\right)-|\nabla \hat{u}|^{2} L \hat{u}\right] d y .
$$

Hence, if we let $\nu=\left(\nu_{1}, \ldots, \nu_{n}\right)$ be the outer unit normal to $B(z, 4 s) \cap \Omega$, then 


$$
\begin{aligned}
\left|\int_{B(z, 4 s) \cap \Omega} \hat{u} L\left(|\nabla \hat{u}|^{2}\right) d y\right| \leqslant & 2\left|\int_{\partial[B(z, 4 s) \cap \Omega]} \sum_{i, j, k=1}^{n} \hat{u} \nu_{i} b_{i j} \hat{u}_{x_{k}} \hat{u}_{x_{k} x_{j}} d H^{n-1}\right| \\
& +\left.\left|\int_{\partial[B(z, 4 s) \cap \Omega]} \sum_{i, j=1}^{n}\right| \nabla \hat{u}\right|^{2} \nu_{i} b_{i j} \hat{u}_{x_{j}} d H^{n-1} \mid \\
& \leqslant c s^{(n-1)} r^{-(p+1)} \hat{u}\left(w^{\prime}\right)^{p+1}
\end{aligned}
$$

where we have used $\left|b_{i j}\right| \leqslant p|\nabla \hat{u}|^{p-2}$, and once again Lemmas $2.5,2.45$ to make estimates. Combining (2.57)-(2.58) letting $\epsilon \rightarrow 0$, and using Fatou's lemma we conclude that Lemma 2.54 is true.

\section{Elliptic measure and a boundary Harnack inequality}

To continue the program outlined in Section 1 , let $\hat{u}, \hat{D}, \hat{z}, \Omega^{+}, \Omega^{-}, R^{+}, R^{-}, \beta$, be as in Section 2. Throughout this section we assume that

$$
R^{+}, R^{-} \in C^{\infty}(\partial B(0,1))
$$

Let $L,\left(b_{i j}\right)$, be as in (1.7), (1.8) relative to $\hat{u}, \hat{D}$. Then from (3.1), Lemmas 2.4, 2.5, see that $\left(b_{i j}\right)$ is symmetric and infinitely differentiable in the closure of $\hat{D}$. Also,

$$
c^{-1}|\nabla u(y)|^{p-2}|\xi|^{2} \leqslant \sum_{i, j=1}^{n} b_{i j}(y) \xi_{i} \xi_{j} \leqslant c|\xi|^{2}|\nabla u(y)|^{p-2}
$$

whenever $y \in \hat{D}$ and $\xi \in \mathbb{R}^{n}$, so $\left(b_{i j}\right)$ is uniformly elliptic in $\hat{D}$. Let $H \subset \hat{D}$ be either a starlike Lipschitz domain or $H=\hat{D}$. Using the above facts and arguing as in [39] we deduce the existence of Green's function, $g(\cdot, \cdot): H \times H \rightarrow(0, \infty]$ with the following properties.

$\left(a^{\prime}\right) \quad g(x, y)=g(y, x)$ when $x \neq y, x, y \in H$,

$\left(b^{\prime}\right) \quad \zeta g(\cdot, y) \in W_{0}^{1,2}[H \backslash \bar{B}(y, \epsilon)]$ whenever $\bar{B}(y, \epsilon) \subset H$ and $\zeta \in C_{0}^{\infty}\left(\mathbb{R}^{n} \backslash \bar{B}(y, \epsilon)\right)$,

$\left.{ }^{\prime}\right) \quad$ if $x \neq y, x, y \in B\left[y, \frac{d(y, \partial \hat{D})}{2}\right] \cap H$, then, $g(x, y) \approx\left(\frac{\hat{u}(y)}{d(y, \partial \hat{D})}\right)^{2-p}|x-y|^{2-n}$

$$
\text { for } n>2 \text { and for } n=2, g(x, y) \approx\left(\frac{\hat{u}(y)}{d(y, \partial \hat{D})}\right)^{2-p} \log \left(\frac{2 d(y, \partial \hat{D})}{|x-y|}\right),
$$

$\left(d^{\prime}\right) \quad$ if $y \in H$, let $g(\cdot, y) \equiv 0$ on $\mathbb{R}^{n} \backslash H$. Then $g(\cdot, y)$ is continuous on $\mathbb{R}^{n} \backslash\{y\}$.

In $\left(c^{\prime}\right)$ we have used Lemma 2.5 to estimate the ellipticity constants in (3.2). Also all proportionality constants depend only on $p, n$, and $\beta$. For given $y \in H$ extend $g(\cdot, y)$ to a continuous function on $\mathbb{R}^{n} \backslash\{y\}$ as in $\left(d^{\prime}\right)$. Then there exists a positive Borel measure, $\omega(\cdot, y)$, on $\mathbb{R}^{n}$ with support in $\partial H, \omega(\partial H, y)=1$, and the property that

$$
\theta(y)=\int \sum_{i, j=1}^{n} \theta_{x_{i}} b_{i j} g_{x_{j}}(\cdot, y) d x+\int \theta d \omega(\cdot, y)
$$

$4^{\text {e }}$ SÉRIE - TOME $40-2007-\mathrm{N}^{\circ} 5$ 
whenever $\theta \in C_{0}^{\infty}\left(\mathbb{R}^{n}\right)$. Observe from (3.4) with $\theta \in C_{0}^{\infty}(H \backslash\{y\})$ that $g(\cdot, y)$ is a weak solution to $L$ in $H \backslash\{y\}$. From Schauder or Moser-DiGiorgi type estimates it follows that $g(\cdot, y)$ is an infinitely differentiable solution to $L$ in $H \backslash\{y\}$ which extends to a continuous function in $\mathbb{R}^{n} \backslash\{y\}$ with $g(\cdot, y) \equiv 0$ on $\mathbb{R}^{n} \backslash H$. Using this fact, we deduce the following interior estimates for $g(\cdot, y)$.

LEMMA 3.5. - If $B(z, 4 s) \subset H \backslash\{y\}$, then,

(a) $s^{2-n} \int_{B(z, s / 2)}|\nabla g(\cdot, y)|^{2} d x \leqslant c \max _{B(z, s)} g(\cdot, y)^{2}$,

(b) $\max _{B(z, s)} g(\cdot, y) \leqslant c \min _{B(z, s)} g(\cdot, y)$,

(c) For some $0<\hat{\alpha}<1,|g(\zeta, y)-g(\xi, y)| \leqslant c\left(\frac{|\zeta-\xi|}{s}\right)^{\hat{\alpha}} \max _{B(z, s)} g(\cdot, y)$ whenever $\zeta, \xi \in B(z, s)$.

Proof. - From Lemma 2.5 and (3.2) we see that the ellipticity constants for $L$ are bounded above and below in $B(z, 2 s)$ by constant multiples of $\left(\frac{u(z)}{d(z, \partial \hat{D})}\right)^{p-2}$ (depending only on $p, n, \beta$ ). Thus Lemma 3.5 follows from standard estimates of the above mentioned authors.

Using (3.4), Lemma 3.5, and arguing as in [39], one can deduce by way of a method of PerronWiener-Brelot, that the continuous Dirichlet problem for $L$ in $H$ has a solution in the sense that given a continuous function $f$ on $\partial H$ there exists a strong solution $F$ to $L$ in $H$ with $F=f$ continuously on $\partial H$. Indeed,

$$
F(y)=\int f d \omega(\cdot, y) \quad \text { for } y \in H .
$$

If $H=\hat{D}$, then from $C^{\infty}$ smoothness of $b_{i j}, \partial \hat{D}$, as well as (3.2) and Schauder type arguments, one can show that $g(\cdot, y)$ extends to a $C^{\infty}$ function in the closure of $\hat{D}$ (away from $y$ ). Using this fact and the divergence theorem we deduce that on $\partial \hat{D}$,

$$
d \omega(\cdot, y)=|\nabla g|^{-1}(\cdot, y) \sum_{i, j=1}^{n} b_{i j} g_{x_{i}}(\cdot, y) g_{x_{j}}(\cdot, y) d H^{n-1} \approx|\nabla \hat{u}|^{p-2}|\nabla g(\cdot, y)| d H^{n-1}
$$

$\omega(\cdot, y)$ is called elliptic measure for $H$ relative to $L$ and $y \in H$. We say that $\omega(\cdot, y)$ is a doubling measure if, for some $1 \leqslant C<\infty$,

$$
\omega(B(x, 2 s), y) \leqslant C \omega(B(x, s), y) \quad \text { whenever } x \in \partial H \text { and } 0<s \leqslant \operatorname{diam} H .
$$

$\omega(\cdot, y)$ is said to be an $A^{\infty}$ weight with respect to $H^{n-1}$ on $\partial H$ if $\omega(\cdot, y)$ is a doubling measure and for some $\delta>0$ there exists $\epsilon=\epsilon(\delta)>0$ with the property that if $E$ is Borel, $E \subset B(x, s) \cap \partial H(x, s$, as in (3.7)), then

$$
\frac{H^{n-1}(E)}{H^{n-1}(B(x, s) \cap \partial H)} \geqslant \delta \longrightarrow \frac{\omega(E, y)}{\omega(B(x, s), y)} \geqslant \epsilon
$$

(see [11] for several equivalent characterizations of $A^{\infty}$ ). Let $\hat{\omega}, \hat{g}$ denote elliptic measure and Green's function for $\hat{D}$. We prove the following result on the elliptic measure. 
Lemma 3.9. - Let $x \in \partial \hat{D}, 0<\rho$, suppose $\hat{z} \notin B(x, 8 \rho)$ and either $B(x, 8 \rho) \cap \partial \Omega^{+}=\emptyset$ or $B(x, 8 \rho) \cap \partial \Omega^{-}=\emptyset$. Then for some $\eta>0$ (depending only on $\left.p, n, \beta\right)$

$$
\hat{\omega}\left(B(x, \rho), a_{\rho}(x)\right) \geqslant \eta .
$$

Proof. - We note that $a_{\rho}(x)$ is defined as in Lemma 2.2 with $r, w$ replaced by $\rho, x$. To prove Lemma 3.9, let $x^{\prime} \in \hat{D}$ be the point on the ray from $\hat{z}$ through $x$ with $\left|x^{\prime}-x\right|=\rho$. Let $\tilde{\Omega}$ be as in Lemmas 2.45, 2.54, defined relative to $x, x^{\prime}, \rho$. From the definition of $\left(b_{i j}\right)$ in (1.8) we find at $y \in \tilde{\Omega}$ that

$$
\sum_{i, j=1}^{n}\left|\nabla b_{i j}\right|^{2} \leqslant c|\nabla \hat{u}|^{2 p-6} \sum_{i, j=1}^{n}\left|\hat{u}_{y_{i} y_{j}}\right|^{2} .
$$

From this display and Lemma 2.54 we conclude that if

$$
d \gamma(y)=\sum_{i, j=1}^{n} d(y, \partial \tilde{\Omega}) \max _{\bar{B}\left(y, \frac{1}{2} d(y, \tilde{\Omega})\right)}\left|\nabla b_{i j}\right|^{2} d y \quad \text { for } y \in \tilde{\Omega},
$$

then $\gamma$ is a Carleson measure on $\tilde{\Omega}$ with

$$
\sup _{0<s<\rho} \frac{\gamma(B(z, s) \cap \tilde{\Omega})}{s^{n-1}} \leqslant c\left(\frac{u\left(x^{\prime}\right)}{\rho}\right)^{2 p-4} \quad \text { whenever } z \in \partial \tilde{\Omega} .
$$

Let $\tilde{\omega}\left(\cdot, x^{\prime}\right)$ be elliptic measure for $\tilde{\Omega}$ defined relative to $L, x^{\prime}$. We shall need the following theorem of [31] tailored to our situation (see also [22, ch. 3]).

THEOREM 3.11. - If (3.10) holds then $\tilde{\omega}\left(\cdot, x^{\prime}\right)$ is an $A^{\infty}$ weight with respect to $H^{n-1}$ with constants $C$ in (3.7) and $\delta, \epsilon$ in (3.8) depending only on $p, n, \beta$.

Proof. - We note that the dependence of the constants in Theorem 3.11 can be easily checked since if

$$
b_{i j}^{\prime}=\left(\frac{u\left(x^{\prime}\right)}{\rho}\right)^{2-p} b_{i j}
$$

then $\tilde{\omega}\left(\cdot, x^{\prime}\right)$ is obviously a solution in $\tilde{\Omega}$ to the PDE one gets from replacing $\left(b_{i j}\right)$ by $\left(b_{i j}^{\prime}\right)$ in the definition of $L$. Moreover ellipticity constants in (3.2) and the Carleson norm in (3.10) taken relative to $\left(b_{i j}^{\prime}\right)$ depend only on $p, n, \beta$.

To get Lemma 3.9 from Theorem 3.11, we first observe from Lemma 2.45 that

$$
H^{n-1}(B(x, \rho) \cap \partial \tilde{\Omega} \cap \partial \hat{D}) \geqslant \rho^{n-1} / c .
$$

In particular there exist $\tilde{x} \in \partial \tilde{\Omega}$ and $\tilde{c}>1$ such that $B(\tilde{x}, \rho / \tilde{c}) \subset B(x, \rho)$ and

$$
H^{n-1}(B(\tilde{x}, \rho / \tilde{c}) \cap \partial \tilde{\Omega} \cap \partial \hat{D}) \geqslant \rho^{n-1} / c .
$$

Applying Theorem 3.11 we deduce from the definition of $A^{\infty}$ in (3.8) that

$$
\tilde{\omega}\left(B(\tilde{x}, \rho / \tilde{c}) \cap \partial \hat{D}, x^{\prime}\right) \geqslant c^{-2} \tilde{\omega}\left(B(\tilde{x}, \rho / \tilde{c}), x^{\prime}\right) \geqslant c^{-3},
$$

$4^{\mathrm{e}}$ SÉRIE - TOME $40-2007-\mathrm{N}^{\circ} 5$ 
where the last inequality follows from the doubling property of $\tilde{\omega}$ and the fact that $\tilde{\omega}\left(\partial \tilde{\Omega}, x^{\prime}\right)=1$. Thus from the maximum principle we have for some $c$

$$
\hat{\omega}\left(B(x, \rho) \cap \partial \tilde{\Omega}, x^{\prime}\right) \geqslant \tilde{\omega}\left(B(x, \rho) \cap \partial \hat{D}, x^{\prime}\right) \geqslant c^{-1} .
$$

We note that Harnack's inequality holds for $\hat{\omega}(\cdot, y)$ with constants depending only on $p, n, \beta$ by the same reasoning as in Lemma 3.5 $(b)$. From Harnack's inequality, we see that $x^{\prime}$ can be replaced by $a_{\rho}(x)$ in the above inequality. Hence Lemma 3.9 is true.

If $x, \rho$ are as in Lemma 3.9 we claim that

$$
\hat{\omega}(B(x, \rho), y) \geqslant c^{-1} \quad \text { whenever } y \in B(x, \rho / 4) \cap \hat{D} .
$$

In fact given $y$ as in (3.12) choose $\hat{y} \in \partial \hat{D}$ with $|y-\hat{y}|=d(y, \partial \hat{D})$. Put $\hat{\rho}=2|y-\hat{y}|$ and observe that Lemma 3.9 can be used with $x, \rho$ replaced by $\hat{y}, \hat{\rho}$. Doing this and using Harnack's inequality we get claim (3.12). Our goal is to prove a boundary Harnack inequality for $L$. Using the argument in [22, ch. 2, sec. 3] or [6, Lemma 2.2], it will turn out that (3.12), (3.3)( $\left.c^{\prime}\right)$, and Harnack's inequality are enough to give this inequality.

Lemma 3.13. - Let $w \in \partial \hat{D}, r>0$. Suppose that $\hat{z} \notin B(w, 8 r)$ and either $B(w, 8 r) \cap$ $\partial \Omega^{-}=\emptyset$ or $B(w, 8 r) \cap \partial \Omega^{+}=\emptyset$. Let $h_{1}, h_{2}$ be positive solutions to $L$ in $\hat{D} \cap B(w, r)$ with $h_{i}=0, i=1,2$, continuously on $\partial \hat{D} \cap B(w, r)$. There exists $\hat{c}$ (depending only on $\left.p, n, \beta\right)$, such that if $\hat{r}=r / \hat{c}$, then

$$
\hat{c}^{-1} \frac{h_{1}\left(a_{\hat{r}}(w)\right)}{h_{2}\left(a_{\hat{r}}(w)\right)} \leqslant \frac{h_{1}(y)}{h_{2}(y)} \leqslant \hat{c} \frac{h_{1}\left(a_{\hat{r}}(w)\right)}{h_{2}\left(a_{\hat{r}}(w)\right)}
$$

whenever $y \in B(w, \hat{r}) \cap \hat{D}$.

Proof. - Let $w^{\prime} \in \hat{D}$ be the point on the ray from $\hat{z}$ through $w$ with $\left|w-w^{\prime}\right|=r / 4$. We assume, as we may, that $h_{i}\left(w^{\prime}\right)=1, i=1,2$, since otherwise we divide $h_{i}$ by these constants for $i=1,2$. We note that interior Hölder continuity estimates and Harnack's inequality can be stated for $h_{1}, h_{2}$ as in Lemma 3.5 with $g(\cdot, y)$ replaced by $h_{1}, h_{2}$. From Harnack's inequality and starlike Lipschitzness of $\hat{D}$ we see that in order to prove Lemma 3.13 it suffices to show for some $c$ that

$$
c^{-1} \leqslant h_{1}(y) / h_{2}(y) \leqslant c \text { for } y \in B(w, r / c) .
$$

To prove (3.14), we note as in (2.46) that if $c^{\prime} \geqslant 10^{10}$ is large enough (depending only on $p, n, \beta$ ), then the domain $\hat{\Omega} \subset B(w, r) \cap \hat{D}$, formed by drawing all open line segments from points in $\partial \hat{D} \cap B\left(w, r / c^{\prime}\right)$ to points in $B\left(w^{\prime}, r / c^{\prime}\right)$, is a starlike Lipschitz domain with center $w^{\prime}$ and Lipschitz constant $\leqslant c(\beta+1)$. Moreover if $\rho=r /\left(c^{\prime}\right)^{2}$, then

$$
B(w, 1000 \rho) \cap \hat{D}=B(w, 1000 \rho) \cap \hat{\Omega} .
$$

With $c^{\prime}$ now fixed let $g^{\prime}$ denote Green's function for $\hat{\Omega}$. If $y \in B(w, \rho / 100) \cap \hat{D}$, choose $\tilde{y} \in \partial \hat{D} \cap \partial \hat{\Omega}$ with $|y-\tilde{y}|=d(y, \partial \hat{\Omega})$. This choice is possible thanks to (3.15). Let $\rho_{m}=2^{-m} \rho$, $\hat{\rho}_{m}=2^{-1 / 2} \rho_{m}$, for $m=0,1,2, \ldots$ and $G_{j}=\bar{B}\left(\tilde{y}, \rho_{j}\right) \cap \partial \hat{D} \backslash B\left(\tilde{y}, \rho_{j+1}\right)$ for $j=0,1,2, \ldots$ From (3.15), (3.3) $\left(c^{\prime}\right),(3.12)$ with $x$ replaced by points in $\partial B\left(\tilde{y}, \hat{\rho}_{0}\right) \cap \partial \hat{D}$, Harnack's inequality, and the weak boundary maximum principle for $L$, we see that

$$
c h_{1}(\cdot) \geqslant\left[\hat{u}\left(a_{\rho}(\tilde{y})\right)\right]^{p-2} r^{n-p} g^{\prime}\left(\cdot, a_{\rho}(\tilde{y})\right) \quad \text { and } \quad h_{2}(\cdot) \leqslant c \hat{\omega}\left(G_{0}, \cdot\right)
$$


in $\left[B(\tilde{y}, \rho / 2) \backslash B\left(a_{\rho}(\tilde{y}), \frac{\left.d\left[a_{\rho}(\tilde{y}), \partial \hat{\Omega}\right)\right]}{1000}\right)\right] \cap \hat{\Omega}$. From (3.16) we conclude that to prove $h_{1}(y) /$ $h_{2}(y) \geqslant c^{-1}$ it suffices to show that

$$
\hat{\omega}\left(G_{0}, y\right) \leqslant c\left[\hat{u}\left(a_{\rho}(\tilde{y})\right)\right]^{p-2} r^{n-p} g^{\prime}\left(y, a_{\rho}(\tilde{y})\right) .
$$

(3.17) is a consequence of the following claim. Given $\epsilon>0$ sufficiently small (depending on $p, n, \beta)$ there exists $c(\epsilon)$ (depending only on $\epsilon, p, n, \beta$ ) such that

$$
\begin{aligned}
& \hat{\omega}\left(G_{j-1}, \cdot\right) \leqslant \epsilon \hat{\omega}\left(G_{j}, \cdot\right)+c(\epsilon)\left[\hat{u}\left(a_{\rho_{j}}(\tilde{y})\right)\right]^{p-2} \rho_{j}^{n-p} g^{\prime}\left(\cdot, a_{\rho_{j}}(\tilde{y})\right) \\
& \quad \text { in } B\left(\tilde{y}, \rho_{j+1}\right) \cap \hat{\Omega}
\end{aligned}
$$

whenever $j=1,2, \ldots$ To prove (3.18) for a fixed $j$, let $\epsilon^{\prime}<10^{-10}$, and let $N$ be the largest positive integer $\leqslant 1 / \epsilon^{\prime}$. Set

$$
E_{i j}=\left\{\zeta \in G_{j}:\left(1+\frac{i-1}{100 N}\right) \hat{\rho}_{j} \leqslant|\zeta-\tilde{y}|<\left(1+\frac{i}{100 N}\right) \hat{\rho}_{j}\right\} \quad \text { for } i=1,2, \ldots, N .
$$

Then

$$
\left\{E_{i j}\right\}_{1}^{N} \text { are pairwise disjoint, } \bigcup_{i=1}^{N} E_{i j} \subset G_{j} \text { and } \hat{\omega}\left(E_{i j}, \cdot\right) \geqslant c^{-1}
$$

on

$$
\begin{aligned}
F_{i j}= & \bigcup\left\{B\left(\zeta, \frac{\epsilon^{\prime} \rho_{j}}{10000}\right) \cap \hat{\Omega}: \zeta \in E_{i j}\right. \text { and } \\
& \left.\left(1+\frac{(i-3 / 4)}{100 N}\right) \hat{\rho}_{j} \leqslant|\zeta-\tilde{y}|<\left(1+\frac{(i-1 / 4)}{100 N}\right) \hat{\rho}_{j}\right\},
\end{aligned}
$$

as we see from (3.12). From (3.19) we find that if

$$
z \in H_{i j}=\left(\hat{\Omega} \cap \partial B\left[\tilde{y},\left(1+\frac{i-1 / 2}{100 N}\right) \hat{\rho}_{j}\right]\right) \backslash F_{i j}
$$

then $d(z, \partial \hat{\Omega}) \geqslant \epsilon^{\prime} \rho_{j} / c$. Using this fact, Harnack's inequality, and (3.3) $\left(c^{\prime}\right)$ once again we find for some $c\left(\epsilon^{\prime}\right)$ that

$$
c\left(\epsilon^{\prime}\right)\left[\hat{u}\left(a_{\rho_{j}}(\tilde{y})\right)\right]^{p-2} \rho_{j}^{n-p} g^{\prime}\left(\cdot, a_{\rho_{j}}(\tilde{y})\right) \geqslant 1 \quad \text { on } H_{i j}
$$

for $1 \leqslant i \leqslant N, j=0,1, \ldots$ Adding (3.19), (3.20) we get that

$$
\hat{\omega}\left(G_{j-1}, \cdot\right) \leqslant 1 \leqslant c \hat{\omega}\left(E_{i j}, \cdot\right)+c\left(\epsilon^{\prime}\right) \hat{u}\left[a_{\rho_{j}}(\tilde{y})\right]^{p-2} \rho_{j}^{n-p} g^{\prime}\left(\cdot, a_{\rho_{j}}(\tilde{y})\right)
$$

on $\partial B\left[\tilde{y},\left(1+\frac{i-1 / 2}{100 N}\right) \hat{\rho}_{j}\right] \cap \hat{\Omega}$. Using the boundary maximum principle for $L$ we deduce that (3.21) is valid in $B\left(\tilde{y}, \rho_{j+1}\right) \cap \hat{\Omega}$. Summing (3.21) for a fixed $j$ from $i=1$ to $N$, we obtain from (3.19) (after division by $N$ ) that

$$
\hat{\omega}\left(G_{j-1}, \cdot\right) \leqslant(c / N) \hat{\omega}\left(G_{j}, \cdot\right)+c\left(\epsilon^{\prime}\right)\left[\hat{u}\left(a_{\rho_{j}}(\tilde{y})\right)\right]^{p-2} \rho_{j}^{n-p} g^{\prime}\left(\cdot, a_{\rho_{j}}(\tilde{y})\right)
$$

$4^{\text {e }}$ SÉRIE - TOME $40-2007-\mathrm{N}^{\circ} 5$ 
in $B\left(\tilde{y}, \rho_{j+1}\right) \cap \hat{\Omega}$. Choose $\epsilon^{\prime}$ so that $c / N<\epsilon$ or equivalently $c \epsilon^{\prime}=\epsilon$ for some $c$. Rewriting (3.22) in terms of $\epsilon$ we obtain claim (3.18) provided $0<\epsilon<1 / c$ and $c$ is large enough.

To prove (3.17) let $k$ be the positive integer with $y \in B\left(\tilde{y}, \rho_{k+1}\right) \backslash \bar{B}\left(\tilde{y}, \rho_{k+2}\right)$. Iterating (3.18) from 1 to $k$ we get

$$
\hat{\omega}\left(G_{0}, y\right) \leqslant \epsilon^{k} \hat{\omega}\left(G_{k}, y\right)+c(\epsilon) \sum_{j=1}^{k} \epsilon^{j-1}\left[\hat{u}\left(a_{\rho_{j}}(\tilde{y})\right)\right]^{p-2} \rho_{j}^{n-p} g^{\prime}\left(y, a_{\rho_{j}}(\tilde{y})\right) .
$$

Observe from $(3.3)\left(a^{\prime}\right)$ that Lemma 3.5 holds with $g(\cdot, y)$ replaced by $g(y, \cdot)$. Thus if

$$
y \notin \bigcup_{i} B\left[a_{\rho_{i}}(\tilde{y}), \frac{d\left(a_{\rho_{i}}(\tilde{y}), \partial \hat{\Omega}\right)}{100}\right],
$$

then from Harnack's inequality applied to $g^{\prime}(y, \cdot), \hat{u}$, we deduce

$$
\epsilon^{j-1}\left[\hat{u}\left(a_{\rho_{j}}(\tilde{y})\right)\right]^{p-2} \rho_{j}^{n-p} g^{\prime}\left(y, a_{\rho_{j}}(\tilde{y})\right) \leqslant c(1 / 2)^{j}\left[\hat{u}\left(a_{\rho}(\tilde{y})\right)\right]^{p-2} \rho^{n-p} g^{\prime}\left(y, a_{\rho}(\tilde{y})\right)
$$

for $j=1,2, \ldots$, provided $\epsilon$ is sufficiently small (depending only on $p, n, \beta$ ). Likewise

$$
\epsilon^{k} \hat{\omega}\left(G_{k}, y\right) \leqslant(1 / 2) \hat{\omega}\left(G_{0}, y\right)
$$

for small $\epsilon$. Fix $\epsilon>0$ so that (3.25), (3.26) are true. Using (3.25), (3.26) in (3.23) we obtain after some arithmetic that (3.17) is valid when (3.24) holds. Otherwise we note the existence of $y^{*}$ satisfying (3.24) (with $y$ replaced by $y^{*}$ ) and with $\left|y^{*}-y\right| \approx d\left(y^{*}, \partial \hat{\Omega}\right) \approx \rho_{k}$. Then (3.17) is valid for $y^{*}$ and from Harnack's inequality it follows that (3.17) is valid for $y$. From the remark above (3.17) we get the lower bound in (3.14). Interchanging $h_{1}, h_{2}$ we also get the upper bound in (3.14). This proves Lemma 3.13.

We end this section by proving the following lemma.

Lemma 3.27. - Let $w \in \partial \hat{D}, r, \hat{r}$ and $h_{1}, h_{2}$ be as in Lemma 3.13. There exist $\lambda, 0<\lambda<1$, and $c^{*}$ (depending only on $p, n, \beta$ ) such that

$$
\left|\frac{h_{1}(y)}{h_{2}(y)}-\frac{h_{1}\left(y^{\prime}\right)}{h_{2}\left(y^{\prime}\right)}\right| \leqslant c^{*}\left(\frac{\left|y-y^{\prime}\right|}{r}\right)^{\lambda} \frac{h_{1}\left(a_{\hat{r}}(w)\right)}{h_{2}\left(a_{\hat{r}}(w)\right)}
$$

whenever $y, y^{\prime} \in B(w, \hat{r} / 4)$.

Proof. - Given $\tilde{y} \in \partial \hat{D} \cap B(w, \hat{r} / 2)$ set

$$
\begin{aligned}
& M(s)=M(s, \tilde{y})=\sup _{B(\tilde{y}, s)} \frac{h_{1}}{h_{2}}(y), \\
& m(s)=m(s, \tilde{y})=\inf _{B(\tilde{y}, s)} \frac{h_{1}}{h_{2}}(y),
\end{aligned}
$$

when $0<s<\hat{r} / 2$, where $\hat{c}$ is the constant in Lemma 3.13. Then $h_{1}-m(s) h_{2} \geqslant 0$ and $M(s) h_{2}-h_{1} \geqslant 0$ in $B(\tilde{y}, s) \cap \hat{D}$. Observe from Harnack's inequality that each of these functions is either positive or identically zero in $B(\tilde{y}, s) \cap \hat{D}$. If both functions are positive then from Lemma 3.13 with $h_{1}, h_{2}$ replaced by $h_{1}-m(s) h_{2}, h_{2}$, and $h_{2} M(s)-h_{1}, h_{2}$, respectively, we 
find that if $\hat{c}$ is the constant in Lemma 3.13 and $\hat{s}=s / \hat{c}$, then

( $\alpha) \quad M(\hat{s})-m(s) \leqslant \hat{c}[m(\hat{s})-m(s)]$,

(ß) $M(s)-m(\hat{s}) \leqslant \hat{c}[M(s)-M(\hat{s})]$.

If $\psi(t)=\psi(t, \tilde{y})=M(t)-m(t)$, then adding $(3.28)(\alpha),(\beta)$ we get

$$
\psi(s)+\psi(\hat{s}) \leqslant \hat{c}[\psi(s)-\psi(\hat{s})]
$$

or

$$
\psi(\hat{s}) \leqslant \frac{\hat{c}-1}{\hat{c}+1} \psi(s) .
$$

Clearly this inequality is also valid if either of the above functions vanishes. If $y, y^{\prime} \in$ $B(w, \hat{r} / 4) \cap \hat{D}$ and $\left|y-y^{\prime}\right|>\frac{\hat{r}}{1000}$, then from Lemma 3.13 we see that Lemma 3.27 holds. If $\left|y-y^{\prime}\right| \leqslant \min \left[\frac{\hat{r}}{1000}, d(y, \partial \hat{D}) / 2\right]$ we can use Harnack's inequality and interior Hölder continuity estimates for $h_{1}, h_{2}$ to get Lemma 3.27 in this case. Otherwise, choose $\tilde{y} \in \partial \hat{D} \cap B(w, \hat{r} / 2)$ with $|y-\tilde{y}|=d(y, \partial \hat{D})$. Iterating (3.29) starting from $s=\hat{r} / 4$ and finishing with $s \approx 4\left|y-y^{\prime}\right|$, we deduce for some $\lambda, 0<\lambda<1$, and $c$,

$$
\begin{aligned}
\left|\frac{h_{1}(y)}{h_{2}(y)}-\frac{h_{1}\left(y^{\prime}\right)}{h_{2}\left(y^{\prime}\right)}\right| & \leqslant M\left(2\left|y-y^{\prime}\right|, \tilde{y}\right)-m\left(2\left|y-y^{\prime}\right|, \tilde{y}\right) \\
& \leqslant c\left(\frac{\left|y-y^{\prime}\right|}{r}\right)^{\lambda} \frac{h_{1}\left(a_{\hat{r}}(w)\right)}{h_{2}\left(a_{\hat{r}}(w)\right)} .
\end{aligned}
$$

Hence Lemma 3.27 holds in all cases.

Remark. - Let $\hat{D}, w, r, \hat{r}$, be as in Lemma 3.27 and suppose that $\hat{\zeta} \in \hat{D} \backslash B(w, 8 r)$. If $h_{1}, h_{2}$ are positive solutions to $L$ in $\hat{D} \backslash\{\hat{\zeta}\}$, then Lemmas 3.13,3.27 are valid with $\hat{r}$ replaced by $r$. Indeed, we can first apply Lemmas 3.13, 3.27, with $w, r$ replaced by $\tilde{y}, \hat{s}=r / 10$, whenever $\tilde{y} \in \partial \hat{D} \cap B(w, r)$. Using $h_{i}\left(a_{\hat{s}}(\tilde{y})\right) \approx h_{i}\left(a_{\hat{r}}(w)\right), i=1,2$, we then get Lemmas 3.13, 3.27 with $\hat{r}$ replaced by $r$.

\section{Deformation of ring domains and proof of Theorem 1}

Let $\Omega_{i}^{-}, \Omega_{i}^{+}, i=1,2$, be starlike Lipschitz domains with center $\hat{z}$ and $\bar{\Omega}_{i}^{-} \subset \Omega_{i}^{+}$for $i=1,2$. Let $R_{i}^{+}, R_{i}^{-}, i=1,2$, be the corresponding graph functions and let $D_{i}=\Omega_{i}^{+} \backslash \bar{\Omega}_{i}^{-}, i=1,2$. For fixed $p, 1<p<\infty$, let $u, v$, be the $p$ capacitary functions for $D_{1}, D_{2}$, respectively. We assume that

$$
R_{i}^{+}, R_{i}^{-} \in C_{0}^{\infty}(\partial B(0,1)) \text { for } i=1,2,
$$

and also that $w \in \partial D_{1} \cap \partial D_{2}, r>0$, with

(a) $B(w, 8 r) \cap D_{1}=B(w, 8 r) \cap D_{2}$.

(b) Either $\bar{B}(w, 8 r) \cap\left[\Omega_{1}^{-} \cup \Omega_{2}^{-}\right]=\emptyset$ or $\bar{B}(w, 8 r) \cap\left[\mathbb{R}^{n} \backslash \Omega_{1}^{+} \cup \mathbb{R}^{n} \backslash \Omega_{2}^{+}\right]=\emptyset$.

We first study properties of the deformation touted above (1.10). To this end we define for $0 \leqslant \tau<1$ and $\omega \in \partial B(0,1)$,

$$
R^{+}(\tau, \omega)=\left[R_{2}^{+}(\omega)\right]^{\tau}\left[R_{1}^{+}(\omega)\right]^{1-\tau}, \quad R^{-}(\tau, \omega)=\left[R_{2}^{-}(\omega)\right]^{\tau}\left[R_{1}^{-}(\omega)\right]^{1-\tau} .
$$

$4^{\mathrm{e}}$ SÉRIE - TOME $40-2007-\mathrm{N}^{\circ} 5$ 
Let $\Omega^{+}(\tau), \Omega^{-}(\tau)$, be starlike Lipschitz domains with center $\hat{z}$ and graph functions, $R^{+}(\tau, \cdot)$, $R^{-}(\tau, \cdot)$, while $D(\tau)=\Omega^{+}(\tau) \backslash \bar{\Omega}^{-}(\tau)$ is the corresponding ring domain. For fixed $p$, $1<p<\infty$, let $u(\cdot, \tau), \tau \in[0,1]$, be the $p$ capacitary function for $D(\tau)$. Extend $u(\cdot, \tau)$ to $\mathbb{R}^{n}$ by putting $u(\cdot, \tau) \equiv 0$ on $\mathbb{R}^{n} \backslash \Omega^{+}(\tau)$ and $u(\cdot, \tau) \equiv 1$ on $\bar{\Omega}^{-}(\tau)$. From Lemma 2.1(c) we see that $\{u(\cdot, \tau), 0 \leqslant \tau \leqslant 1\}$ are Hölder continuous on $\mathbb{R}^{n}$ with exponent independent of $\tau \in[0,1]$. From Lemma 2.5 and the same argument as in the proof of Lemma 2.4, we see that $u(\cdot, \tau), \tau \in[0,1]$, has an infinitely differentiable extension to $\bar{D}(\tau)$. Moreover, a check of the argument shows that there exists $\delta, \epsilon>0$, for a given nonnegative integer $k$ and fixed $s \in[0,1]$, such that

(-) $\quad \min _{\bar{D}(\tau)}|\nabla u(\cdot, \tau)|>\epsilon$ provided $\tau \in(s-\delta, s+\delta)$,

$(--) \quad$ the $C^{k}$ norm of $u(\cdot, \tau)$ in $\bar{D}(\tau)$ is uniformly bounded for $\tau \in(s-\delta, s+\delta)$.

Using Ascoli's theorem on $\mathbb{R}^{n},(4.3)$, and uniqueness of $u(\cdot, \tau)$ we see that if $\partial^{l} u(\cdot, \tau)$ denotes an arbitrary $l$-th partial derivative of $u(\cdot, \tau)$ in $D(\tau)$ (with respect to $x$ ), then for $s \in[0,1]$,

$$
\lim _{\tau \rightarrow s} \partial^{l} u(\cdot, \tau)=\partial^{l} u(\cdot, s) \quad \text { as } \tau \rightarrow s, \text { uniformly on compact subsets of } D(s) .
$$

Next we consider regularity in $\tau$.

LEMMA 4.5. - Let $u(\cdot, \cdot)$ be as above. Then $u_{\tau}(x, \tau)=\frac{\partial}{\partial \tau} u(x, \tau)$ exists continuously whenever $(x, \tau) \in \bigcup_{t \in[0,1]} D(t) \times\{t\}$. Moreover for $\tau \in[0,1], u_{\tau}(\cdot, \tau)$ extends continuously to $\bar{D}(\tau)$ and

$(\alpha) u_{\tau}(\cdot, \tau)$ is a solution to $(1.7)$ in $D(\tau)$ with $b_{i j}$ defined relative to $u(\cdot, \tau)$,

( $\beta) u_{\tau}(y, \tau)=-\langle y-\hat{z}, \nabla u(y, \tau)\rangle \log \left(R_{2}^{\prime} / R_{1}^{\prime}\right)\left(\frac{y-\hat{z}}{|y-\hat{z}|}\right)$ when $y \in \partial D(\tau)$, where ${ }^{\prime}=+$ if $y \in \partial \Omega^{+}(\tau)$ and ${ }^{\prime}=-$ if $y \in \partial \Omega^{-}(\tau)$.

( $\gamma) \log \left(\frac{v(x)-a}{u(x)-a}\right)=\int_{0}^{1} \frac{u_{\tau}(x, \tau)}{u(x, \tau)-a} d \tau$ whenever $a=0,1$ and $x \in B(w, 8 r) \cap D_{1}$.

Proof. - We assume, as we may, that $\hat{z}=0$. If $\tau=0,1$ in Lemma 4.5, then $u_{\tau}$ should be interpreted as a limit of one-sided difference quotients. To begin, if $\xi=\left(\xi_{1}, \ldots, \xi_{n}\right)$, $w=\left(w_{1}, \ldots, w_{n}\right) \in \mathbb{R}^{n} \backslash\{0\}$, and $1 \leqslant i \leqslant n$ we note that

$$
\begin{aligned}
|\xi|^{p-2} \xi_{i}-|w|^{p-2} w_{i} & =\int_{0}^{1} \frac{d}{d \lambda}\left\{|\lambda \xi+(1-\lambda) w|^{p-2}\left[\lambda \xi_{i}+(1-\lambda) w_{i}\right]\right\} d \lambda \\
& =\sum_{j=1}^{n}(\xi-w)_{j}\left(\int_{0}^{1} a_{i j}[\lambda \xi+(1-\lambda) w] d \lambda\right),
\end{aligned}
$$

where for $1 \leqslant i, j \leqslant n$,

$$
a_{i j}(\eta)=|\eta|^{p-4}\left[(p-2) \eta_{i} \eta_{j}+\delta_{i j}|\eta|^{2}\right] \quad \text { for } \eta \in \mathbb{R}^{n} \backslash\{0\} .
$$

In this display $\delta_{i j}$, once again, denotes the Kronecker delta. Using (4.3), (4.4), and (1.2), we deduce for given $s \in[0,1]$ that if

$$
U(x)=U(x, \tau, s)=\frac{u(x, s)-u(x, \tau)}{s-\tau},
$$




$$
A_{i j}(x)=A_{i j}(x, \tau, s)=\int_{0}^{1} a_{i j}[\lambda \nabla u(x, s)+(1-\lambda) \nabla u(x, \tau)] d \lambda,
$$

then, whenever $x \in D(\tau) \cap D(s), \tau \in[s-\delta, s+\delta]$, and $1 \leqslant i, j \leqslant n$,

$$
\tilde{L} U(x)=\sum_{i, j=1}^{n} \frac{\partial}{\partial x_{i}}\left[A_{i j}(x) U_{x_{j}}\right]=0 \quad \text { on } D(\tau) \cap D(s) .
$$

Moreover, if $x \in D(\tau) \cap D(s)$, then it is easily seen that

$$
\text { (*) }\left.\quad c^{-1}|\xi|^{2}|| \nabla u(x, s)|+| \nabla u(x, \tau)\right|^{p-2} \leqslant \sum_{i, j=1}^{n} A_{i j}(x) \xi_{i} \xi_{j}
$$
whenever $\xi \in \mathbb{R}^{n} \backslash\{0\}$,

$$
(* *) \quad \sum_{i, j=1}^{n}\left|A_{i j}(x)\right| \leqslant c|| \nabla u(x, s)|+| \nabla u(x, \tau) \|^{p-2}
$$

where $c$ depends only on $p, n$.

Given $\epsilon_{1}>0$ small, choose $\delta_{1}, \delta_{2}>0$ so small that

$$
\begin{aligned}
& \left|\nabla u(t \omega, \tau)-\nabla u\left[\left(1-\delta_{1}\right) R^{+}(s, \omega) \omega, \tau\right]\right| \\
& \quad+\left|\nabla u(\hat{t} \omega, \tau)-\nabla u\left[\left(1+\delta_{1}\right) R^{-}(s, \omega) \omega, \tau\right]\right| \leqslant \epsilon_{1}
\end{aligned}
$$

whenever $t \in\left[\left(1-\delta_{1}\right) R^{+}(s, \omega), R^{+}(\tau, \omega)\right], \hat{t} \in\left[R^{-}(\tau, \omega),\left(1+\delta_{1}\right) R^{-}(s, \omega)\right]$, and $\omega \in \partial B(0,1)$, $\tau \in\left(s-\delta_{2}, s+\delta_{2}\right)$. Also, we require

$$
|\nabla u(y, s)-\nabla u(y, \tau)|<\epsilon_{1} \quad \text { on } \Gamma_{1} \cup \Gamma_{2} \text { if } \tau \in\left(s-\delta_{2}, s+\delta_{2}\right)
$$

where

$$
\begin{aligned}
& \Gamma_{1}=\left\{\left(1-\delta_{1}\right) R^{+}(s, \omega) \omega: \omega \in \partial B(0,1)\right\}, \\
& \Gamma_{2}=\left\{\left(1+\delta_{1}\right) R^{-}(s, \omega) \omega: \omega \in \partial B(0,1)\right\} .
\end{aligned}
$$

This choice of $\delta_{1}, \delta_{2}>0$ is possible thanks to (4.3), (4.4). Let $y \in \partial[D(s) \cap D(\tau)]$, for some $\tau \in\left(s-\delta_{2}, s+\delta_{2}\right)$ with $y /|y|=\omega$. Let $a_{1}=\min \left\{R^{\prime}(\tau, \omega), R^{\prime}(s, \omega)\right\}$ and $a_{2}=$ $\max \left\{R^{\prime}(\tau, \omega), R^{\prime}(s, \omega)\right\}$ where $^{\prime}=+$ if $y$ lies in the unbounded component of $D(\tau) \cap D(s)$ while $^{\prime}=-$ if $y$ is in the bounded component of $D(\tau) \cap D(s)$. We note that

$$
U(y, \tau, s)= \pm \int_{a_{1}}^{a_{2}} \frac{\partial}{\partial t} U(t \omega, \tau, s) d t
$$

where the - sign is taken if ${ }^{\prime}=+$ and the + sign if ${ }^{\prime}=-$. Using this note (4.9), (4.10), and the chain rule, we deduce that

$$
U(y, \tau, s)=\frac{R^{\prime}(\tau, \omega)-R^{\prime}(s, \omega)}{s-\tau}\left\{e+\left[R^{\prime}(s, \omega)\right]^{-1}\langle\nabla u(y, s), y\rangle\right\}
$$

where $|e| \leqslant 3 \epsilon_{1}$. Since

$$
R_{s}^{\prime}(s, \omega)=\left[R^{\prime}(s, \omega)\right] \log \left(R_{2}^{\prime} / R_{1}^{\prime}\right)(\omega) \quad \text { for }^{\prime} \in\{+,-\},
$$

$4^{\mathrm{e}}$ SÉRIE - TOME $40-2007-\mathrm{N}^{\circ} 5$ 
we conclude from (4.11) that $U(\cdot, \tau, s)$ converges uniformly on $\partial[D(\tau) \cap D(s)]$ to the right-hand side of Lemma $4.5(\beta)$ as $\tau \rightarrow s$ in the sense that given $\epsilon_{2}>0$, there is a $\delta_{3}>0$ such that

$$
\left|U(y, \tau, s)+\langle y, \nabla u(y, s)\rangle \log \left(R_{2}^{\prime} / R_{1}^{\prime}\right)\left(\frac{y}{|y|}\right)\right| \leqslant \epsilon_{2}
$$

for all $y \in \partial[D(\tau) \cap D(s)]$ and $\tau \in\left(s-\delta_{3}, s+\delta_{3}\right)$. Let $\psi$ be a classical solution to

$$
L \psi=\sum_{i, j=1}^{n} \frac{\partial}{\partial x_{i}}\left[b_{i j}(x) \frac{\partial \psi}{\partial x_{j}}\right]=0 \quad \text { in } D(s)
$$

with continuous boundary values

$$
\psi(y)=-\langle y, \nabla u(y, s)\rangle \log \left(R_{2}^{\prime} / R_{1}^{\prime}\right)\left(\frac{y}{|y|}\right) \quad \text { on } \partial D(s) .
$$

Here $b_{i j}(x)=a_{i j}(\nabla u(x, s)), 1 \leqslant i, j \leqslant n, x \in D(s)$, and $a_{i j}$ are as in (4.6). Since $\left(b_{i j}\right)$ are uniformly elliptic it follows from the usual minimizing argument that

$$
\int_{D(s)}|\nabla \psi|^{2} d x \leqslant M<\infty .
$$

To prove Lemma $4.5(\alpha),(\beta)$, we need to show that $\psi=u_{s}(\cdot, s)$. To this end, we observe from (4.3), (4.4), (4.8) that $A_{i j}(\cdot, s, t), 1 \leqslant i, j \leqslant n$, are uniformly elliptic and $C^{l}, l=1,2, \ldots$ with ellipticity and norm constants in $D(s) \cap D(\tau)$ that are independent of $\tau \in\left(s-\delta_{3}, s+\delta_{3}\right)$. Also, from (4.12), (4.7), (4.8), and the boundary maximum principle for $\tilde{L}$ we see that the functions $U(\cdot, \tau, s)$ are uniformly bounded in $D(s) \cap D(\tau), \tau \in\left(s-\delta_{3}, s+\delta_{3}\right)$.

Using the above facts and Schauder theory, we find that if $\lim _{k \rightarrow \infty} \tau_{k}=s$, then a subsequence of $\left\{U\left(\cdot, \tau_{k}, s\right)\right\}$ converges in the $C^{l}$ norm on each compact subset of $D(s)$ for $l=0,1,2, \ldots$ Let $V$ be such a limit. From these remarks and (4.7), (4.8), we deduce that $L V=0$ in $D(s)$ where $L$ is as in (4.13). Let

$$
\begin{gathered}
\phi_{1}(x)=\max \left(U(x, \tau, s)-\psi(x)-2 \epsilon_{2}, 0\right), \\
\phi_{2}(x)=\min \left(U(x, \tau, s)-\psi(x)+2 \epsilon_{2}, 0\right)
\end{gathered}
$$

when $x \in D(s) \cap D(\tau)$ and $\tau \in\left(s-\delta_{3}, s+\delta_{3}\right)$. From (4.12) we see that $\phi_{k}, k=1,2$, vanishes continuously on $\partial[D(s) \cap D(\tau)]$. This fact and (4.7) imply

$$
\int_{D(s) \cap D(\tau)} \sum_{i, j=1}^{n} A_{i j} U_{x_{j}}\left(\phi_{k}\right)_{x_{i}} d x=0=\int_{D(s) \cap D(\tau)} \sum_{i, j=1}^{n} b_{i j} \psi_{x_{j}}\left(\phi_{k}\right)_{x_{i}} d x
$$

for $k=1,2$. This equality, (4.3), and (4.8) yield for some $0<K<\infty$, depending on various smoothness constants but independent of $s, \tau \in\left(s-\delta_{3}, s+\delta_{3}\right)$, that

$$
\begin{aligned}
& K^{-1} \int_{D(s) \cap D(\tau) \cap\left\{|U-\psi|>2 \epsilon_{2}\right\}}|\nabla U(\cdot, \tau, s)-\nabla \psi|^{2} d x \\
& \leqslant \int_{D(s) \cap D(\tau)} \sum_{i, j=1}^{n} A_{i j}\left(U_{x_{j}}-\psi_{x_{j}}\right)\left(\phi_{1}+\phi_{2}\right)_{x_{i}} d x
\end{aligned}
$$




$$
=\int_{D(s) \cap D(\tau)} \sum_{i, j=1}^{n}\left(b_{i j}-A_{i j}\right) \psi_{x_{j}}\left(\phi_{1}+\phi_{2}\right)_{x_{i}} d x .
$$

Using Cauchy's inequality with $\epsilon^{\prime} s,(4.3),(4.4),(4.8),(4.15)$, it follows for some $K_{1}$ having the same dependence as $K$ that

$\int_{D(s) \cap D(\tau) \cap\left\{|U-\psi|>2 \epsilon_{2}\right\}}|\nabla U(\cdot, \tau, s)-\nabla \psi|^{2} d x \leqslant K_{1} \int_{D(s) \cap D(\tau)} \sum_{i, j=1}^{n}\left|b_{i j}-A_{i j}\right|^{2}|\nabla \psi|^{2} d x \rightarrow 0$

as $\tau \rightarrow s$. Using Fatou's lemma and Poincaré's inequality, we conclude that

$$
\begin{gathered}
\int_{D(s) \cap D(\tau) \cap\left\{|V-\psi|>2 \epsilon_{2}\right\}}|V-\psi|^{2} d x \\
\leqslant \liminf _{k \rightarrow \infty} \int_{D(s) \cap D(\tau) \cap\left\{|U-\psi|>2 \epsilon_{2}\right\}}\left|U\left(\cdot, \tau_{k}, s\right)-\psi\right|^{2} d x=0 .
\end{gathered}
$$

Since $\epsilon_{2}>0$ is arbitrary we deduce from (4.16) that $\psi \equiv V$. Since every subsequence of $\{U(\cdot, \tau, s)\}$ must converge to $\psi$ we conclude that $u_{s} \equiv \psi$. Thus Lemma $4.5(\alpha),(\beta)$, holds. Continuity of $u_{s}$ as in Lemma 4.5, is a consequence of (4.3), (4.4), Lemma 4.5( $\left.\alpha\right),(\beta)$, and Schauder theory. Finally Lemma $4.5(\gamma)$ follows from continuity of $u_{s}$, the fact that $0<u(\cdot, s)<1$ in $D(s)$, the fundamental theorem of calculus, and the observation that (4.2) yields, $x \in D(s), s \in[0,1]$, whenever $x \in B(w, 8 r) \cap D_{1}$. The proof of Lemma 4.5 is now complete.

We now consider some applications of Lemma 4.5. We show that under certain deformations, a boundary Harnack inequality applies to the integrand in Lemma $4.5(\gamma)$ for each fixed $\tau$. Thus we will essentially obtain Theorem 2 in certain special cases. To prove Theorem 2, we shall deform $\hat{D}_{1}$ into $\hat{D}_{2}$ by a series of such deformations, ending in Section 5 . At the end of this section we pause to deduce Theorem 1 from Lemma 4.34. To begin suppose that $D=\Omega^{+} \backslash \Omega^{-}$ is a starlike Lipschitz ring domain with Lipschitz constant $\beta$, center $\hat{z}$, and graph functions $R^{+}, R^{-}$. Let $u$ be the $p$ capacitary function for $D$. As in Theorem 2 we assume for $w \in \partial D$, $r>0$, that

$$
\hat{z} \notin B(w, 16 r) \text { and either } \bar{B}(w, 8 r) \cap \Omega^{-}=\emptyset \text { or } \bar{B}(w, 8 r) \cap \mathbb{R}^{n} \backslash \Omega^{+}=\emptyset .
$$

We prove

Lemma 4.18. - Given $p, 1<p<\infty$, let $D, R^{+}, R^{-}, u$, be as above. Let $w, r, \hat{z}$ satisfy (4.17) and suppose that $w \in \partial \Omega^{+}$. If $R^{+}, R^{-} \in C^{\infty}(\partial B(0,1))$, then there exists a starlike Lipschitz ring domain $D^{*}=\Omega^{+} \backslash B(\hat{z}, \rho)$ with $\rho \approx d\left(\hat{z}, \partial \Omega^{+}\right)$and the following properties:

(i) $\bar{B}(w, 8 r) \cap B(\hat{z}, 2 \rho)=\emptyset$ and $B(\hat{z}, 2 \rho) \subset \Omega^{+}$.

(j) If $u^{*}$ denotes the $p$ capacitary function for $D^{*}$, then for some $c$

$$
c^{-1} \frac{u^{*}\left(a_{r}(w)\right)}{u\left(a_{r}(w)\right)} \leqslant \frac{u^{*}(z)}{u(z)} \leqslant c \frac{u^{*}\left(a_{r}(w)\right)}{u\left(a_{r}(w)\right)} \text { whenever } z \in B(w, r / 2) \cap D .
$$

(k) Let $u^{*}$ be as in $(j)$ and $w_{1}, w_{2} \in B(w, r / 2) \cap D$. Then there exist $c, \lambda, 0<\lambda<1$, such that

$$
\left|\frac{u^{*}\left(w_{1}\right) / u\left(w_{1}\right)}{u^{*}\left(w_{2}\right) / u\left(w_{2}\right)}-1\right|+\left|\frac{u\left(w_{1}\right) / u^{*}\left(w_{1}\right)}{u\left(w_{2}\right) / u^{*}\left(w_{2}\right)}-1\right| \leqslant c\left(\frac{\left|w_{1}-w_{2}\right|}{r}\right)^{\lambda} .
$$

$4^{\text {e }}$ SÉRIE - TOME $40-2007-\mathrm{N}^{\circ} 5$ 
Moreover if (i) holds with $\rho$ replaced by $\tilde{\rho}$ and $\tilde{u}$ is the $p$ capacitary function for $\tilde{D}=$ $\Omega^{+} \backslash B(\hat{z}, \tilde{\rho})$, then $(j),(k)$ are valid with either $u, D$ or $u^{*}, D^{*}$ replaced by $\tilde{u}, \tilde{D}$.

Proof. - In Lemma 4.18, $\lambda, c$ and the proportionality constant for $\rho / d\left(\hat{z}, \partial \Omega^{+}\right)$depend only on $p, n, \beta$. In the proof of Lemma 4.18 we assume, as usual, that $\hat{z}=0$. We consider two cases. First suppose that

$$
\bar{\Omega}^{-} \cap \partial B\left(0, d\left(0, \partial \Omega^{+}\right) / 4\right) \neq \emptyset .
$$

In this case since $\Omega^{-}$is starlike Lipschitz, we can choose $c^{\prime}$, depending only on $p, n, \beta$, to be the largest number such that if $\rho=d\left(0, \partial \Omega^{+}\right) / c^{\prime}$, then

$$
B(0,2 \rho) \subset \Omega^{-} .
$$

(i) of Lemma 4.18 follows from (4.17), (4.20). Let $R_{2}^{-}(\omega)=\rho \omega$ when $\omega \in \partial B(0,1)$ and set $R_{i}^{+}=R^{+}$for $i=1,2, R_{1}^{-}=R^{-}$. Applying the deformation at the beginning of this section, with $D_{1}=D, D_{2}=D^{*}$, we get $u(\cdot, \tau)$, the $p$ capacitary function for $D(\tau)=\Omega^{+}(\tau) \backslash \bar{\Omega}^{-}(\tau)$. From (4.20) and starlike Lipschitzness of $\Omega^{+}$we see that $2 \leqslant R^{-} / \rho \leqslant c$. This inequality, Lemma 4.5( $\beta)$, and Lemma 2.5(i) imply that $u_{\tau} \equiv 0$ on $\partial \Omega^{+}$and

$$
0<-u_{\tau} \leqslant-c\langle x, \nabla u(x)\rangle
$$

for $x \in \partial \Omega^{-}(\tau)$. Since $u_{\tau},\langle x, \nabla u(x, \tau)\rangle$, both satisfy (1.7), (1.8) with $\left(b_{i j}\right)$ defined relative to $u(\cdot, \tau)$ in $D(\tau)$ it follows from the weak maximum principle for this divergence form equation that (4.21) also holds in $D(\tau)$. Let

$$
r(\tau)=\sup \left\{s: \bar{B}(w, 2 s) \cap \Omega^{-}(\tau)=\emptyset\right\} .
$$

From (4.20), (4.17), and the definition of $R(\tau, \cdot)$, we see that $r$ is increasing and continuous on $[0,1]$ with $r(0) \geqslant 4 r, r(1) \geqslant \rho$. Next we note that Lemmas 3.13, 3.27 can be applied with $h_{1}, h_{2}$ replaced by $-u_{\tau}(\cdot, \tau), u(\cdot, \tau)$ and $\hat{D} \cap B(w, 8 r)$ by $D(\tau) \cap B(w, 2 r(\tau))$. Using this note, Remark at the end of Section 3, and Lemma 4.5 $(\gamma)$ we get for $w_{1}, w_{2} \in B(w, r)$,

$$
\begin{aligned}
\left|\log \left(\frac{u^{*}\left(w_{1}\right)}{u\left(w_{1}\right)}\right)-\log \left(\frac{u^{*}\left(w_{2}\right)}{u\left(w_{2}\right)}\right)\right| & \leqslant \int_{0}^{1}\left|\frac{u_{\tau}\left(w_{1}, \tau\right)}{u\left(w_{1}, \tau\right)}-\frac{u_{\tau}\left(w_{2}, \tau\right)}{u\left(w_{2}, \tau\right)}\right| d \tau \\
& \leqslant c\left(\frac{\left|w_{1}-w_{2}\right|}{r}\right)^{\lambda} \int_{0}^{1} \frac{-u_{\tau}\left(a_{r(\tau)}(w), \tau\right)}{u\left(a_{r(\tau)}(w), \tau\right)} d \tau
\end{aligned}
$$

where $a_{r(\tau)}(w)$ is defined relative to $D(\tau)$. If $r(0) \geqslant \rho / 1000$, we deduce from Lemma 2.5 that

$$
\frac{-u_{\tau}\left(a_{r(\tau)}(w), \tau\right)}{u\left(a_{r(\tau)}(w), \tau\right)} \leqslant c
$$

Using (4.23) in (4.22) we find for $w_{1}, w_{2} \in B(w, r)$ that

$$
\left|\log \left(\frac{u^{*}\left(w_{1}\right)}{u\left(w_{1}\right)}\right)-\log \left(\frac{u^{*}\left(w_{2}\right)}{u\left(w_{2}\right)}\right)\right| \leqslant c\left(\frac{\left|w_{1}-w_{2}\right|}{r}\right)^{\lambda} .
$$

ANNALES SCIENTIFIQUES DE L'ÉCOLE NORMALE SUPÉRIEURE 
Choosing $w_{1}=a_{r}(w) \in D \cap B(w, r)$, and exponentiating (4.24) we conclude that $(j)$ of Lemma 4.18 is valid. ( $k$ ) follows from $(j),(4.24)$, and the fact that $|\log t| /|t-1|$ is bounded below for $t \in(0, c]$ by a constant depending only on $p, n, \beta$. If $r(0)<\rho / 1000$ we choose, $\tau_{1}, \tau_{2}, \ldots, \tau_{m} \in[0,1]$, so that $\tau_{i}, 1 \leqslant i \leqslant m$, is the value in $[0,1]$ with $r\left(\tau_{i}\right)=2^{i} r(0), 1 \leqslant i \leqslant m$, and $\rho / 1000 \leqslant r\left(\tau_{m}\right)<\rho / 500$. Also let $\tau_{m+1}=1$ and note that $\tau_{1}=0$ by definition. We claim that

$$
0<\tau_{i+1}-\tau_{i} \leqslant \operatorname{cr}\left(\tau_{i}\right) / \rho \text { for } 1 \leqslant i \leqslant m
$$

To prove this claim observe from the definition of $r(\tau)$ that there exists $\omega_{i}, 1 \leqslant i \leqslant m$, such that $\omega_{i} \in \partial B(0,1)$ and $R^{-}\left(\tau_{i}, \omega_{i}\right) \omega_{i} \in \partial B\left(w, r\left(\tau_{i}\right)\right)$ for $i=1,2, \ldots, m$. From the definition of $\tau_{i}$ we see that

$$
R^{-}\left(\tau_{i}, \omega_{i}\right) / R^{-}\left(\tau_{i+1}, \omega_{i+1}\right) \leqslant e^{c r\left(\tau_{i}\right) / \rho} .
$$

On the other hand from the definition of $R^{-}(\tau, \cdot)$ and the definition of $\tau_{i}$ we deduce that

$$
\begin{aligned}
\frac{R^{-}\left(\tau_{i}, \omega_{i}\right)}{R^{-}\left(\tau_{i+1}, \omega_{i+1}\right)} & =\left[R^{-}\left(\omega_{i+1}\right) / \rho\right]^{\left(\tau_{i+1}-\tau_{i}\right)} \cdot\left[R^{-}\left(\omega_{i+1}\right) / R^{-}\left(\omega_{i}\right)\right]^{\left(\tau_{i}-1\right)} \\
& \geqslant e^{-c r\left(\tau_{i}\right) / \rho} 2^{\left(\tau_{i+1}-\tau_{i}\right)}
\end{aligned}
$$

Combining (4.26), (4.27) and then taking logarithms, we get claim (4.25).

Observe from Lemma 2.5 and (4.21) that

$$
\frac{-u_{\tau}\left(a_{r\left(\tau_{i}\right)}(w), \tau\right)}{u\left(a_{r\left(\tau_{i}\right)(w), \tau}\right)} \leqslant \frac{c \rho}{r\left(\tau_{i}\right)} \quad \text { whenever } \tau \in\left[\tau_{i}, \tau_{i+1}\right], 1 \leqslant i \leqslant m .
$$

Applying Lemmas 3.13, 3.27, as above, and using (4.25), (4.28) we find for $w_{1}, w_{2} \in$ $B(w, r) \cap D$ that

$$
\begin{aligned}
\int_{\tau_{i}}^{\tau_{i+1}}\left|\frac{u_{\tau}\left(w_{1}, \tau\right)}{u\left(w_{1}, \tau\right)}-\frac{u_{\tau}\left(w_{2}, \tau\right)}{u\left(w_{2}, \tau\right)}\right| d \tau & \leqslant c\left(\frac{\left|w_{1}-w_{2}\right|}{r\left(\tau_{i}\right)}\right)^{\lambda} \int_{\tau_{i}}^{\tau_{i+1}} \frac{-u_{\tau}\left(a_{r\left(\tau_{i}\right)}(w), \tau\right)}{u\left(a_{r\left(\tau_{i}\right)}(w), \tau\right)} d \tau \\
& \leqslant c 2^{-i \lambda}\left(\frac{\left|w_{1}-w_{2}\right|}{r(0)}\right)^{\lambda} \leqslant c 2^{-i \lambda}\left(\frac{\left|w_{1}-w_{2}\right|}{r}\right)^{\lambda} .
\end{aligned}
$$

From (4.29) and Lemma 4.5( $\gamma)$ we conclude for $w_{1}, w_{2} \in B(w, r)$ that

$$
\begin{aligned}
\left|\log \left(\frac{u^{*}\left(w_{1}\right)}{u\left(w_{1}\right)}\right)-\log \left(\frac{u^{*}\left(w_{2}\right)}{u\left(w_{2}\right)}\right)\right| & \leqslant \sum_{i=1}^{m} \int_{\tau_{i}}^{\tau_{i+1}}\left|\frac{u_{\tau}\left(w_{1}, \tau\right)}{u\left(w_{1}, \tau\right)}-\frac{u_{\tau}\left(w_{2}, \tau\right)}{u\left(w_{2}, \tau\right)}\right| d \tau \\
& \leqslant c\left[\left(\sum_{i=1}^{m-1} 2^{-i \lambda}\right)+1\right]\left(\frac{\left|w_{1}-w_{2}\right|}{r}\right)^{\lambda} .
\end{aligned}
$$

From (4.30) we see first that (4.24) is valid and second that Lemma 4.18 holds for our choice of $\rho$ when (4.19) is true.

If (4.19) is false, choose $t$ to be the largest number in $[0,1]$ with

$$
\{x: u(x) \geqslant t\} \cap \partial B\left(0, d\left(0, \partial \Omega^{+}\right) / 4\right) \neq \emptyset .
$$

$4^{\text {e }}$ SÉRIE - TOME $40-2007-\mathrm{N}^{\circ} 5$ 
Then from Lemma 2.5 we see that $\min (u / t, 1)$ is the $p$ capacitary function for the starlike Lipschitz ring domain, $\tilde{D}=\{x: 0<u(x)<t\}$ with Lipschitz constant depending only on $p, n, \beta$. Let $\rho$ be the largest number so that $B(0,2 \rho) \subset\{x: u(x)>t\}$. We can now repeat our earlier argument with $\tilde{D}, u / t$ replacing $u, D$. Doing this we get Lemma 4.18 for $u / t$. Clearly we can replace $u / t$ by $u$ in $(j),(k)$ of this lemma. The proof of the first part of Lemma 4.18 is now complete.

If $\tilde{\rho}$ is as in Lemma 4.18 , we can use Lemma 4.5 to deform $D^{*}$ into $\tilde{D}$. Using the above argument with either $B(\hat{z}, \rho)$ or $B(\hat{z}, \tilde{\rho})$ replacing $\Omega^{-}$we get Lemma 4.18 for $u^{*}, \tilde{u}$. Clearly Lemma 4.18 for $u, u^{*}$ and $u^{*}, \tilde{u}$ implies Lemma 4.18 for $u, \tilde{u}$.

Next we state an analogue of Lemma 4.18 when $w \in \partial \Omega^{-}$.

Lemma 4.32. - Given $p, 1<p<\infty$, let $D, R^{+}, R^{-}$, $u$, be as in Lemma 4.18. Let $w, r, \hat{z}$ satisfy (4.17) and suppose that $w \in \partial \Omega^{-}$. If $R^{+}, R^{-} \in C^{\infty}(\partial B(0,1))$, then there exists a starlike Lipschitz ring domain $D^{*}=B(\hat{z}, \hat{\rho}) \backslash \Omega^{-}$with $\hat{\rho} \approx d(\hat{z}, D)$ and the following properties:

$\left(i^{\prime}\right) \bar{B}(w, 8 r) \cap B(\hat{z}, \hat{\rho} / 2)=\emptyset$ and $\Omega^{-} \subset B(\hat{z}, \hat{\rho} / 2)$.

$\left(j^{\prime}\right)$ If $u^{*}$ denotes the $p$ capacitary function for $D^{*}$ and $u_{+}=1-u, u_{*}=1-u^{*}$, then for some $c$,

$$
c^{-1} \frac{u_{*}\left(a_{r}(w)\right)}{u_{+}\left(a_{r}(w)\right)} \leqslant \frac{u_{*}(z)}{u_{+}(z)} \leqslant c \frac{u_{*}\left(a_{r}(w)\right)}{u_{+}\left(a_{r}(w)\right)} \quad \text { whenever } z \in B(w, r / 2) \cap D .
$$

$\left(k^{\prime}\right)$ Let $u_{*}, u_{+}$, be as in $\left(j^{\prime}\right)$ and $w_{1}, w_{2} \in B(w, r / 2) \cap D$. Then there exist $c, \lambda, 0<\lambda<1$, such that

$$
\left|\frac{u_{*}\left(w_{1}\right) / u_{+}\left(w_{1}\right)}{u_{*}\left(w_{2}\right) / u_{+}\left(w_{2}\right)}-1\right|+\left|\frac{u_{+}\left(w_{1}\right) / u_{*}\left(w_{1}\right)}{u_{+}\left(w_{2}\right) / u_{*}\left(w_{2}\right)}-1\right| \leqslant c\left(\frac{\left|w_{1}-w_{2}\right|}{r}\right)^{\lambda} .
$$

Moreover if $\left(i^{\prime}\right)$ holds with $\rho$ replaced by $\tilde{\rho}$ and $\tilde{u}$ is the $p$ capacitary function for $\tilde{D}=$ $B(\hat{z}, \tilde{\rho}) \backslash \Omega^{-}$, then $\left(j^{\prime}\right),\left(k^{\prime}\right)$ are valid with either $u_{+}, D$ or $u_{*}, D^{*}$ replaced by $1-\tilde{u}, \tilde{D}$. Constants have the same dependence as in Lemma 4.18. If

Proof. - Let $s^{-}=\max _{\partial B(0,1)} R^{-}$and $s^{+}=\max _{\partial B(0,1)} R^{+}$. Again one considers two cases.

$$
\bar{B}\left(\hat{z}, 4 s^{-}\right) \cap \partial \Omega^{+} \neq \emptyset
$$

put $\hat{\rho}=2 s^{+}$and define $D^{*}$ as in Lemma 4.32. Arguing as in the case when (4.19) of Lemma 4.18 held, we obtain $\left(i^{\prime}\right),\left(j^{\prime}\right),\left(k^{\prime}\right)$ of Lemma 4.32. If (4.33) is false we choose $t$ to be the smallest number in $[0,1]$ with $\{x: 0<u(x) \leqslant t\} \cap \bar{B}\left(\hat{z}, 4 s^{-}\right) \neq \emptyset$. In this case we put $\hat{D}=\{x: t<$ $u(x)<1\}, \hat{u}=\max \{(u-t) /(1-t), 0\}$ and note from Lemma 2.5 that $\hat{D}$ is starlike Lipschitz with Lipschitz constant depending only on $p, n, \beta$. Let $\tilde{s}=\max \{|x-\hat{z}|: x \in \partial \hat{D}\}$ and set $\hat{\rho}=2 \tilde{s}$. Define $D^{*}, u^{*}$ as previously relative to $\hat{\rho}$. Proceeding as in the first case with $u_{+}, D$, replaced by $1-\hat{u}=\frac{1-u}{1-t}, \hat{D}$, we get Lemma 4.32 for $1-\hat{u}, u_{*}$. Since $\left(j^{\prime}\right),\left(k^{\prime}\right)$ are unchanged upon multiplying a given function by a constant, we conclude that the first part of Lemma 4.32 holds in this case also. To prove the second part of Lemma 4.32 we use Lemma 4.5 to deform $D^{*}$ into $\tilde{D}$ and argue as in the first part of this lemma.

LEMMA 4.34. - Let $\Omega_{i}^{+}, i=1,2$, be starlike Lipschitz domains with center $\hat{z}$ and $C^{\infty}$ graph functions, $R_{i}^{+}$. Let $D_{i}=\Omega_{i}^{+} \backslash \bar{B}\left(\hat{z}, \rho_{1}\right)$ and suppose for fixed $p, 1<p<\infty$, that $u_{i}$ is the $p$ capacitary function for $D_{i}, i=1,2$. If $w \in \partial \Omega_{1}^{+} \cap \partial \Omega_{2}^{+}, r, \rho_{1}>0$, assume that 


$$
\begin{aligned}
& B(w, 8 r) \cap \Omega_{1}^{+}=B(w, 8 r) \cap \Omega_{2}^{+}, \quad B(w, 8 r) \cap B\left(\hat{z}, 2 \rho_{1}\right)=\emptyset, \\
& B\left(\hat{z}, 2 \rho_{1}\right) \subset \Omega_{i}^{+} \quad \text { for } i=1,2 .
\end{aligned}
$$

Then $(j),(k)$ of Lemma 4.18 are valid with $u, u^{*}$ replaced by $u_{1}, u_{2} . c, \lambda$ depend only on $p, n$ and the Lipschitz constants for $D_{i}, i=1,2$.

Proof. - As usual we assume that $\hat{z}=0$. We employ the same strategy as in the proof of Lemma 4.18. Applying the deformation at the beginning of Section 4 , we get $u(\cdot, \tau), \tau \in(0,1)$, satisfying Lemma 4.5. For fixed $\tau \in[0,1]$ let $g(\cdot, y)$ be Green's function for $D(\tau)$ and the operator $L$ in (1.7) with pole at $y \in D(\tau)$. Here $\left(b_{i j}\right)$ are defined relative to $u(\cdot, \tau)$. Then $u(\cdot, \tau), g(\cdot, y)$ have $C^{\infty}$ extensions to $\partial D(\tau)$ and since $\nabla g, \nabla u$ are both parallel to the inner normal at a given point of $\partial D(\tau)$, we have

$$
\sum_{i, j=1}^{n} b_{i j} g_{x_{i}} g_{x_{j}}=(p-1)|\nabla u|^{p-2}|\nabla g(\cdot, y)|^{2} \quad \text { on } \partial D(\tau)
$$

Let

$$
E_{j}=\partial \Omega^{+}(\tau) \cap\left[B\left(w, 2^{j} r\right) \backslash B\left(w, 2^{j-1} r\right)\right] \text { for } j=4,5, \ldots, m,
$$

where $m$ is the first positive integer with $E_{j}=\emptyset$ for $j>m$. If $y \in D(\tau)$, let

$$
I_{j}(y)=-(p-1) \int_{E_{j}}\langle x, \nabla u(x, \tau)\rangle \log \left(R_{2}^{+} / R_{1}^{+}\right)\left(\frac{x}{|x|}\right)|\nabla u(x, \tau)|^{p-2}|\nabla g(x, y)| d H^{n-1}(x) .
$$

From (4.35), (3.6), and Lemma 4.5 we see that

$$
u_{\tau}(y, \tau)=\sum_{j=4}^{m} I_{j}(y) \quad \text { whenever } y \in D(\tau) .
$$

Let $\beta_{i}$ be the Lipschitz constant for $D_{i}, i=1,2$, and set $\hat{r}=\sup \left\{s: B(w, 2 s) \cap \bar{B}\left(0, \rho_{1}\right)=\emptyset\right\}$. We note that

$$
\hat{r} \approx d\left(0, \partial \Omega^{+}(\tau)\right) \approx \operatorname{diam} D(\tau)
$$

with proportionality constants depending only on $\beta_{1}, \beta_{2}, p, n$. From (4.37) and Lipschitzness of $\log R_{i}^{+}, i=1,2$, we find that

$$
\left|\log \left(R_{2}^{+} / R_{1}^{+}\right)\right| \leqslant c 2^{j} r / \hat{r} \quad \text { on } E_{j}, 4 \leqslant j \leqslant m .
$$

Let $k$ be the largest positive integer with $E_{k} \subset B(w, \hat{r})$. From Lemmas 3.13, 3.27, and (3.3) $\left(c^{\prime}\right)$, we observe for $\zeta \in D(\tau)$ near a point of $E_{j}, 4 \leqslant j \leqslant k$, and $w_{1}, w_{2} \in B(w, r) \cap D_{1}$, as in Remark at the end of Section 3, that

$$
\begin{aligned}
& \left|\frac{g\left(\zeta, w_{1}\right)}{u(\zeta, \tau) u\left(w_{1}, \tau\right)}-\frac{g\left(\zeta, w_{2}\right)}{u(\zeta, \tau) u\left(w_{2}, \tau\right)}\right| \leqslant c\left(\frac{\left|w_{1}-w_{2}\right|}{2^{j} r}\right)^{\lambda} \frac{g\left(\zeta, a_{2^{j} r}(w)\right)}{u(\zeta, \tau) u\left(a_{2^{j} r}(w), \tau\right)} \\
& \quad \leqslant c\left(\frac{\left|w_{1}-w_{2}\right|}{2^{j} r}\right)^{\lambda}\left(2^{j(p-n)} r^{p-n}\right) u\left(a_{2^{j} r}(w), \tau\right)^{-p}
\end{aligned}
$$

$4^{\mathrm{e}}$ SÉRIE - TOME $40-2007-\mathrm{N}^{\circ} 5$ 
A similar argument yields (4.39) with $a_{2^{j} r}(w)$ replaced by $a_{\hat{r}}(w)$ when $k<j \leqslant m$. Letting $\zeta \rightarrow x \in E_{j}$ we obtain from (4.39) and smoothness of $u(\cdot, \tau), g$ that

$$
\begin{aligned}
& \left|\frac{\left|\nabla g\left(x, w_{1}\right)\right|}{|\nabla u(x, \tau)| u\left(w_{1}, \tau\right)}-\frac{\left|\nabla g\left(x, w_{2}\right)\right|}{|\nabla u(x, \tau)| u\left(w_{2}, \tau\right)}\right| \\
& \quad \leqslant c\left(\frac{\left|w_{1}-w_{2}\right|}{2^{j} r}\right)^{\lambda}\left(2^{j} r\right)^{p-n} u\left(a_{2^{j} r}(w), \tau\right)^{-p}
\end{aligned}
$$

when $4 \leqslant j \leqslant k$. If $k<j \leqslant m$, we replace $a_{2^{j} r}(w)$ by $a_{\hat{r}}(w)$ in (4.40). We can now use (4.40), (4.38), (4.37) to estimate $I_{j}$. If $4 \leqslant j \leqslant k$ and $w_{1}, w_{2} \in B(w, r) \cap D$ we find

$$
\begin{aligned}
& \left|\frac{I_{j}\left(w_{1}\right)}{u\left(w_{1}, \tau\right)}-\frac{I_{j}\left(w_{2}\right)}{u\left(w_{2}, \tau\right)}\right| \\
& \quad \leqslant c 2^{j} r \int_{E_{j}}|\nabla u(x, \tau)|^{p}\left|\frac{\left|\nabla g\left(x, w_{1}\right)\right|}{|\nabla u(x, \tau)| u\left(w_{1}, \tau\right)}-\frac{\left|\nabla g\left(x, w_{2}\right)\right|}{|\nabla u(x, \tau)| u\left(w_{2}, \tau\right)}\right| d H^{n-1}(x) \\
& \quad \leqslant c\left(\int_{E_{j}}|\nabla u|^{p} d x\right)\left(\frac{\left|w_{1}-w_{2}\right|}{2^{j} r}\right)^{\lambda}\left(2^{j(p+1-n)} r^{p+1-n}\right) u\left(a_{2^{j} r}(w), \tau\right)^{-p} .
\end{aligned}
$$

From Lemma 2.39 and Lemma $2.35(b)$ we deduce for $1 \leqslant j \leqslant k$ that

$$
\int_{E_{j}}|\nabla u|^{p} d H^{n-1} \leqslant c u\left(a_{2^{j}} r(w), \tau\right)^{p}\left(2^{j} r\right)^{n-1-p} .
$$

Putting (4.42) in (4.41) it follows that

$$
\left|\frac{I_{j}\left(w_{1}\right)}{u\left(w_{1}, \tau\right)}-\frac{I_{j}\left(w_{2}\right)}{u\left(w_{2}, \tau\right)}\right| \leqslant c 2^{-j \lambda}\left(\frac{\left|w_{1}-w_{2}\right|}{r}\right)^{\lambda} .
$$

A similar argument yields that (4.43) also holds for $k<j \leqslant m$. Note that $m-k \leqslant c$, thanks to (4.37). From this note, (4.36), (4.43) we see that

$$
\left|\frac{u_{\tau}\left(w_{1}, \tau\right)}{u\left(w_{1}, \tau\right)}-\frac{u_{\tau}\left(w_{2}, \tau\right)}{u\left(w_{2}, \tau\right)}\right| \leqslant \sum_{j=4}^{m}\left|\frac{I_{j}\left(w_{1}\right)}{u\left(w_{1}, \tau\right)}-\frac{I_{j}\left(w_{2}\right)}{u\left(w_{2}, \tau\right)}\right| \leqslant c\left(\frac{\left|w_{1}-w_{2}\right|}{r}\right)^{\lambda} .
$$

Using (4.44) and (4.5) $(\gamma)$ as in (4.22) we conclude that for $w_{1}, w_{2} \in B(w, r) \cap D$,

$$
\left|\log \left(\frac{u_{2}\left(w_{1}\right)}{u_{1}\left(w_{1}\right)}\right)-\log \left(\frac{u_{2}\left(w_{2}\right)}{u_{1}\left(w_{2}\right)}\right)\right| \leqslant c\left(\frac{\left|w_{1}-w_{2}\right|}{r}\right)^{\lambda} .
$$

(4.45) is easily seen to imply Lemma 4.34 (see the discussion after (4.24)).

Next we state a version of Lemma 4.34 when $w \in \partial \Omega_{1}^{-} \cap \partial \Omega_{2}^{-}$.

LEMMA 4.46. - Let $\Omega_{i}^{-}, i=1,2$, be starlike Lipschitz ring domains with center $\hat{z}$ and $C^{\infty}$ graph functions, $R_{i}^{-}$. Let $D_{i}=B\left(0, \rho_{1}\right) \backslash \bar{\Omega}_{i}^{-}$and suppose for fixed $p, 1<p<\infty$, that $u_{i}$ is the 
p capacitary function for $D_{i}, i=1,2$. If $w \in \partial \Omega_{1}^{-} \cap \partial \Omega_{2}^{-}, r, \rho_{1}>0$, assume that

$$
B(w, 8 r) \cap \Omega_{1}^{-}=B(w, 8 r) \cap \Omega_{2}^{-}, \quad \hat{z} \notin B(w, 16 r), \Omega_{i}^{-} \subset B\left(\hat{z}, \rho_{1} / 2\right), i=1,2 .
$$

Then $\left(j^{\prime}\right),\left(k^{\prime}\right)$ of Lemma 4.32 are valid with $u_{+}, u_{*}$, replaced by $1-u_{1}, 1-u_{2} . c, \lambda$ depend only on $p, n$ and the Lipschitz constants for $D_{i}, i=1,2$.

Proof. - The proof of Lemma 4.46 is essentially identical to the proof of Lemma 4.34. We omit the details.

Proof of Theorem 1. - We now use Lemma 4.34 to prove Theorem 1. First we claim that

$$
\begin{aligned}
& \text { Lemma } 4.34 \text { remains true without the assumption that } \\
& \qquad R_{i}^{+} \in C^{\infty}(\partial B(0,1)), i=1,2 .
\end{aligned}
$$

Indeed, let $\left\{R_{i, m}^{+}\right\}, i=1,2, m=1,2, \ldots$, be a sequence of $C^{\infty}(\partial B(0,1))$ real valued functions with

(a) $\| R_{i, m}^{+} \hat{\|}_{\partial B(0,1)} \leqslant c \beta_{i} \quad$ for $i=1,2, m=1,2, \ldots$.

(b) $R_{i, m}^{+} \rightarrow R_{i}^{+} \quad$ uniformly on $\partial B(0,1)$.

(c) $R_{1, m}^{+}=R_{2, m}^{+}$on $\left\{\frac{x-\hat{z}}{|x-\hat{z}|}: x \in B(w, 8 r-1 / m)\right\} \cap \partial D$.

In (4.48)(a), $c$ depends only on $n$. Existence of $\left\{R_{i, m}^{+}\right\}$satisfying (4.48) is easily shown. For example extend $R_{i}^{+}, i=1,2$ to $\mathbb{R}^{n} \backslash\{\hat{z}\}$ by putting $R_{i}^{+}(x)=R_{i}^{+}\left(\frac{x-\hat{z}}{|x-\hat{z}|}\right)$ when $x \in \mathbb{R}^{n} \backslash\{\hat{z}\}$. One can take $\log R_{i, m}^{+}=\log R_{i}^{+} * \theta_{\epsilon_{m}}$ for certain $\epsilon_{m}>0, m=1,2, \ldots$, with $\lim _{m \rightarrow \infty} \epsilon_{m}=0$. Here $\theta \in C_{0}^{\infty}[B(0,1)]$ with $\int_{\mathbb{R}^{n}} \theta d x=1$ and $\theta_{\epsilon}(x)=\epsilon^{-n} \theta(x / \epsilon), x \in \mathbb{R}^{n}$. Also, $*$ denotes convolution on $\mathbb{R}^{n}$. Let $\Omega_{i, m}^{+}$be the starlike Lipschitz domain with center $\hat{z}$ and graph function $R_{i, m}^{+}$for $i=1,2, m=1,2, \ldots$ Put $D_{i, m}=\Omega_{i, m}^{+} \backslash B\left(\hat{z}, \rho_{1}\right)$ and let $u_{i, m}$ be the $p$ capacitary functions for $D_{i, m}$. From (4.48) and Lemma 2.2 we see that $u_{i, m}, D_{i, m}$ converge to $u_{i}, D_{i}$ in Hölder and Hausdorff distance norms, as $m \rightarrow \infty$. We apply Lemma 4.34 with $u_{i}, D_{i}, 8 r$ replaced by $u_{i, m}, D_{i, m}, 8 r-1 / m$. Since the constants in Lemma 4.34 depend only on $p, n$ and the Lipschitz constants for $D_{i, m}$ we can let $m \rightarrow \infty$ and conclude that (4.47) is true.

Let $\tilde{u}, \tilde{v}, r, w, \phi, G$ be as in Theorem 1 and let $\tilde{w}=w+\frac{r}{4} e_{n}$. As in (2.46), observe that if $c^{\prime}$ is large enough (depending on $p, n$ and the Lipschitz norm of $\phi$ ), then the domain $\Omega \subset G \cap B(w, r)$ obtained from drawing all open line segments from points in $B\left(w, r / c^{\prime}\right) \cap \partial G$ to points in $B\left(\tilde{w}, r / c^{\prime}\right)$ is Lipschitz starlike with center $\tilde{w}$ and Lipschitz constant $\leqslant c\left(\||\nabla \phi|\|_{\infty}+1\right)$, where $c$ depends only on $n$. Let $r^{\prime}=\frac{r}{4 c^{\prime}}$ and let $u$ be the $p$ capacitary function for $D=\Omega \backslash \bar{B}\left(\tilde{w}, r^{\prime} / 4\right)$. Since $\tilde{u}(\tilde{w})=1=\tilde{v}(\tilde{w})$, it follows from Harnack's inequality and the weak maximum principle for the $p$ Laplacian that

$$
c \min (\tilde{u}, \tilde{v}) \geqslant u \text { in } D
$$

Let

$$
\begin{gathered}
R(\omega)=|y-\tilde{w}| \quad \text { when } \omega=\frac{y-\tilde{w}}{|y-\tilde{w}|} \text { and } y \in B\left(w, r^{\prime}\right) \cap \partial G \\
K_{i}=\left\{\frac{y-\tilde{w}}{|y-\tilde{w}|}: y \in B\left(w, 2^{-i} r^{\prime}\right)\right\} \quad \text { for } i=0,1,2 \\
L=\sup _{K_{0}} R .
\end{gathered}
$$

$4^{\mathrm{e}}$ SÉRIE - TOME $40-2007-\mathrm{N}^{\circ} 5$ 
From our construction, we observe for some $c$ (depending on $p, n$, and the Lipschitz constant for $\phi$ ) that

$$
\min \left\{d\left(K_{2}, \partial B(0,1) \backslash K_{1}\right), d\left(K_{1}, \partial B(0,1) \backslash K_{0}\right)\right\} \geqslant c^{-1} .
$$

Let $0 \leqslant \alpha \leqslant 1, \alpha \in C_{0}^{\infty}\left(\mathbb{R}^{n}\right)$, with $\alpha \equiv 1$ on $K_{2}$ and $\alpha \equiv 0$ on $\partial B(0,1) \backslash K_{1}$. We can choose $\alpha$ so that

$$
|\nabla \alpha| \leqslant c^{-1}
$$

thanks to (4.50). Let

$$
\log R^{\prime}(\omega)= \begin{cases}\alpha \log R+(1-\alpha) \log (2 L) & \text { when } \omega \in K_{0}, \\ \log (2 L) & \text { when } \omega \in \partial B(0,1) \backslash K_{0}\end{cases}
$$

Using (4.51) it is easily shown that

$$
\| \log R^{\prime} \hat{\|}_{\partial B(0,1)} \leqslant c\left(\| \log R \hat{\|}_{\partial K_{0}}+1\right) .
$$

Let $\Omega^{\prime}$ be the starlike Lipschitz domain with center at $\tilde{w}$ and graph function $R^{\prime}$. Let $D^{\prime}=$ $\Omega^{\prime} \backslash B\left(\tilde{w}, r^{\prime} / 4\right)$ and let $u^{\prime}$ be the $p$ capacitary function for $D^{\prime}$. Then from our construction, the fact that $L \geqslant c r^{\prime}$, Lemma 2.2, and Harnack's inequality we deduce first that $c u^{\prime} \geqslant \max (\tilde{u}, \tilde{v})$ on $G \cap \partial B\left(w, 3 r^{\prime} / 4\right)$ and second from the weak maximum principle that $c u^{\prime} \geqslant \max (\tilde{u}, \tilde{v})$ in $G \cap B\left(w, 3 r^{\prime} / 4\right)$. Using this inequality and (4.49) we conclude that to prove Theorem 1 it suffices to prove this theorem for $u, u^{\prime}$. Now from our construction it is easily checked that the hypotheses of Lemma 4.34 are satisfied with $u_{1}, u_{2}, r$ replaced by $u, u^{\prime}, r^{\prime} / 100$ so Theorem 1 holds for $u, u^{\prime}$. From our earlier reasoning we conclude that Theorem 1 is true.

\section{Proof of Theorems 2,3 , and 4}

Let $\hat{D}_{i}, i=1,2$, be as in Theorem 2 with centers $\hat{x}, \hat{y}$ and corresponding $p$ capacitary functions $\hat{u}_{i}, i=1,2$. We note that Lemmas 4.18, 4.34 imply the validity of Theorem 2 when $w \in \partial \hat{D}_{i}$, $i=1,2$, lies in the unbounded components of $\mathbb{R}^{n} \backslash \hat{D}_{i}, i=1,2$, and $\hat{x}=\hat{y}$ (i.e. $\hat{D}_{i}, i=1,2$ have the same center). Moreover, Lemmas 4.32, 4.46 imply the validity of Theorem 2 when $w \in \partial \hat{D}_{i}$ lies in the bounded components of $\mathbb{R}^{n} \backslash \hat{D}_{i}, i=1,2$, and $\hat{x}=\hat{y}$. Indeed, the $C^{\infty}$ smoothness assumption on the various graph functions in the above lemmas can be done away with as in (4.48). Also, if $w$ lies in the unbounded components of $\mathbb{R}^{n} \backslash \hat{D}_{i}, i=1,2$, and $\hat{x}=\hat{y}$, then we can first use Lemma 4.18 to reduce the proof of Theorem 2 to the case when $\hat{D}_{i}=\hat{\Omega}_{i}^{+} \backslash \bar{B}\left(\hat{x}, \rho_{1}\right)$, $i=1,2$, where $\partial \hat{\Omega}_{i}^{+}$is the outer boundary of $\hat{D}_{i}$. Applying Lemma 4.34 we then get Theorem 2 . A similar argument holds when $w$ lies in the bounded components of $\mathbb{R}^{n} \backslash \hat{D}_{i}, i=1,2$, and $\hat{x}=\hat{y}$. Thus

$$
\text { Theorem } 2 \text { is valid when } \hat{D}_{1}, \hat{D}_{2} \text { have the same center. }
$$

To complete the proof of Theorem 2 we need to consider the case when $\hat{x} \neq \hat{y}$. To this end recall that $w \in \partial \hat{D}_{1} \cap \partial \hat{D}_{2}$ with

$$
B(w, 2 r) \cap \hat{D}_{1}=B(w, 2 r) \cap \hat{D}_{2} .
$$

Also, $B(w, 8 r)$ does not contain any points in either both bounded components or both unbounded components of $\mathbb{R}^{n} \backslash \hat{D}_{i}, i=1,2$. Once again we consider two cases. If $w$ lies in 
the unbounded component of $\mathbb{R}^{n} \backslash \hat{D}_{i}$ for $i=1,2$, let $\hat{R}_{i}^{+}$be the corresponding graph functions for the outer boundary of $\hat{D}_{i}, i=1,2$. Put $\hat{\Omega}_{1}^{+}=\left\{\hat{x}+t \hat{R}_{1}^{+}(\omega) \omega: \omega \in \partial B(0,1), 0 \leqslant t<1\right\}$ and $\hat{\Omega}_{2}^{+}=\left\{\hat{y}+t \hat{R}_{2}^{+}(\omega) \omega: \omega \in \partial B(0,1), 0 \leqslant t<1\right\}$. From Lemma 4.18 we see that since $w \in \partial \hat{\Omega}_{1}^{+} \cap \partial \hat{\Omega}_{2}^{+}$we may also assume that

$$
\begin{gathered}
\hat{D}_{1}=\hat{\Omega}_{1}^{+} \backslash \bar{B}\left(\hat{x}, \rho_{1}\right), \quad \hat{D}_{2}=\hat{\Omega}_{2}^{+} \backslash \bar{B}\left(\hat{y}, \rho_{2}\right), \\
\text { where } \rho_{1} \approx d\left(\hat{x}, \partial \hat{\Omega}_{1}^{+}\right), \rho_{2} \approx d\left(\hat{y}, \partial \hat{\Omega}_{2}^{+}\right) .
\end{gathered}
$$

Moreover,

$$
\bar{B}(w, 8 r) \cap\left[B\left(\hat{x}, 2 \rho_{1}\right) \cup B\left(\hat{y}, 2 \rho_{2}\right)\right]=\emptyset .
$$

Given $z \in B(w, r) \cap \partial \hat{D}_{i}, i=1,2$, we claim that it suffices to show there exists $c$, depending only on $p, n$, and the Lipschitz constants for $\hat{\Omega}_{i}^{+}, i=1,2$, such that if $z \in B(w, r) \cap \partial \hat{\Omega}_{i}^{+}, i=1,2$, then

$$
\text { the conclusion of Theorem } 2 \text { holds with } w, r \text { replaced by } z, r / c \text {. }
$$

In fact assuming (5.5) one can cover $B(w, r) \cap \partial \hat{D}_{i}, i=1,2$, by at most $c^{+}$such balls to get Theorem 2 near $B(w, r) \cap \partial \hat{D}_{1}$. One can then use interior Hölder continuity and Harnack estimates for $\hat{u}_{1}, \hat{u}_{2}$ (see Lemma 2.1) to get Theorem 2 for the rest of $B(w, r) \cap \hat{D}_{1}$. Hence we prove only (5.5). Let $z \in B(w, r) \cap \partial \hat{\Omega}_{i}^{+}, i=1,2$, and let $l$ denote the open line segment drawn from $\hat{x}$ to $z$. Let $\beta_{1}$ be the Lipschitz constant for $\hat{D}_{1}$. If $\zeta \in l$, let $\Omega=\Omega(\zeta)$, be the domain obtained from drawing all open line segments connecting points in $B\left(\zeta,|\zeta-z| / c^{\prime}\right)$ to points in $B\left(z,|\zeta-z| / c^{\prime}\right) \cap \partial \hat{\Omega}_{1}^{+}$. If $c^{\prime}$ is large enough (depending only on $\left.p, n, \beta_{1}\right)$ and $|\zeta-z| \geqslant r / 8$, it follows from the same reasoning as in (2.46) that $\Omega$ is starlike Lipschitz with center at $\zeta$, constant $\leqslant c\left(\beta_{1}+1\right)$, and $\Omega \subset \hat{D}_{1}$. Let $D(\zeta)=\Omega(\zeta) \backslash B\left(\zeta, \frac{1}{2}|\zeta-z| / c^{\prime}\right)$. We note that if $\xi \in B\left(\zeta, \frac{1}{4}|\zeta-z| / c^{\prime}\right)$, then

$$
D(\zeta) \text { is also a starlike Lipschitz ring with center at } \xi \text { and constant } \leqslant c\left(\beta_{1}+1\right) .
$$

Let $\zeta_{1} \in l$ be such that $\left|\zeta_{1}-z\right|=r / 8$. From our construction it is easily seen that there exists $0<\theta<1 / 2$ (depending only on $p, n, \beta_{1}$ ) and a sequence $\left\{\zeta_{i}\right\}_{1}^{N}$ with

$$
\begin{aligned}
& \text { (a) } \frac{\left|\zeta_{i+1}-z\right|}{\left|\zeta_{i}-z\right|} \geqslant 1+\theta, \quad 1 \leqslant i \leqslant N-1 \\
& \text { (b) } \zeta_{i+1} \in B\left(\zeta_{i}, \frac{1}{4}\left|\zeta_{i}-z\right| / c^{\prime}\right) \text { for } 1 \leqslant i \leqslant N-1 \text { and } \hat{x} \in B\left(\zeta_{N}, \frac{1}{4}\left|\zeta_{N}-z\right| / c^{\prime}\right) .
\end{aligned}
$$

Let $v_{i}, i=1,2, \ldots, N$, be the $p$ capacitary function for $D\left(\zeta_{i}\right)$. From (5.6) we see that $D\left(\zeta_{i}\right), D\left(\zeta_{i+1}\right)$ are both starlike Lipschitz with respect to $\zeta_{i+1}$. Also $v_{1} \approx v_{2} \approx 1$ at a point in $D\left(\zeta_{i}\right) \cap D\left(\zeta_{i+1}\right)$ with distance $\approx\left|\zeta_{i}-z\right|$ from $\partial \hat{D}_{i}, i=1,2$. From these facts, (5.1), (5.6), and our construction we see that Theorem 2 can be applied with $\hat{u}_{1}, \hat{u}_{2}, r, w$ replaced by $v_{i}, v_{i+1}, \frac{\left|\zeta_{i}-z\right|}{100 c^{\prime}}, z$ to get first, for $c \geqslant 10^{6} c^{\prime}$, that $v_{j} / v_{k} \leqslant c$ on $B\left(z,\left|\zeta_{i}-z\right| / c\right) \cap \hat{D}_{1}$ whenever $j, k \in\{i, i+1\}$ and thereupon, from (5.7), that

$$
\begin{aligned}
& \left|\log \left(\frac{v_{i+1}\left(w_{1}\right)}{v_{i}\left(w_{1}\right)}\right)-\log \left(\frac{v_{i+1}\left(w_{2}\right)}{v_{i}\left(w_{2}\right)}\right)\right| \\
& \quad \leqslant c\left(\frac{\left|w_{1}-w_{2}\right|}{\left|\zeta_{i}-z\right|}\right)^{\lambda} \leqslant c(1+\theta)^{-i \lambda}\left(\frac{\left|w_{1}-w_{2}\right|}{r}\right)^{\lambda}
\end{aligned}
$$

$4^{\text {e }}$ SÉRIE - TOME $40-2007-\mathrm{N}^{\circ} 5$ 
whenever $w_{1}, w_{2} \in B(z, r / c) \cap \hat{D}_{1}$. Summing this inequality we deduce that

$$
\begin{aligned}
& \left|\log \left(\frac{v_{N}\left(w_{1}\right)}{v_{1}\left(w_{1}\right)}\right)-\log \left(\frac{v_{N}\left(w_{2}\right)}{v_{1}\left(w_{2}\right)}\right)\right| \\
& \quad \leqslant \sum_{i=1}^{N-1}\left|\log \left(\frac{v_{i+1}\left(w_{1}\right)}{v_{i}\left(w_{1}\right)}\right)-\log \left(\frac{v_{i+1}\left(w_{2}\right)}{v_{i}\left(w_{2}\right)}\right)\right| \leqslant c\left(\frac{\left|w_{1}-w_{2}\right|}{r}\right)^{\lambda} .
\end{aligned}
$$

In view of (5.1), (5.6), (5.7), we see that Theorem 2 can also be applied to $v_{N}, \hat{u}_{1}$. Doing this and using (5.9) we get

$$
\left|\log \left(\frac{\hat{u}_{1}\left(w_{1}\right)}{v_{1}\left(w_{1}\right)}\right)-\log \left(\frac{\hat{u}_{1}\left(w_{2}\right)}{v_{1}\left(w_{2}\right)}\right)\right| \leqslant c\left(\frac{\left|w_{1}-w_{2}\right|}{r}\right)^{\lambda}
$$

whenever $w_{1}, w_{2} \in B(z, r / c) \cap \hat{D}_{1}$ and $c$ is large enough, depending only on $p, n, \beta_{1}$.

Let $\beta_{2}$ be the Lipschitz constant for $\hat{D}_{2}$ and let $\eta$ be a point in $\hat{D}_{2}$ on the line segment from $\hat{y}$ to $z$ with $|\eta-z|=r / 8$. Let $H(\eta)$ be the domain obtained from drawing all infinite rays with tips in $B\left(\eta,|\eta-z| / c^{\prime}\right)$ through points in $B\left(z,|\eta-z| / c^{\prime}\right) \cap \partial \hat{\Omega}_{2}^{+}$. Let $\Omega(\eta)=H(\eta) \cap \hat{D}_{2}$. If $c^{\prime}$ is large enough, it follows as in (2.46) or the construction of $\Omega(\zeta)$, that $\Omega(\eta)$ is the union of all open line segments joining points in $B\left(\eta,|\eta-z| / c^{\prime}\right)$ to points in $B\left(z,|\eta-z| / c^{\prime}\right) \cap \partial \hat{\Omega}_{2}^{+}$. Thus $\Omega(\eta)$ is starlike with respect to centers in $\bar{B}\left(\eta, \frac{1}{16} r / c^{\prime}\right)$ and starlike Lipschitz with respect to points in $B\left(\eta, \frac{1}{32} r / c^{\prime}\right)$. Again $c^{\prime}$ and the Lipschitz constant can be chosen to depend only on $p, n, \beta_{2}$. Let $D(\eta)=\Omega(\eta) \backslash B\left(\eta, \frac{1}{16} r / c^{\prime}\right)$ and let $v$ be the $p$ capacitary function for $D(\eta)$. Arguing as in the proof of (5.10) we deduce that

$$
\left|\log \left(\frac{\hat{u}_{2}\left(w_{1}\right)}{v\left(w_{1}\right)}\right)-\log \left(\frac{\hat{u}_{2}\left(w_{2}\right)}{v\left(w_{2}\right)}\right)\right| \leqslant c\left(\frac{\left|w_{1}-w_{2}\right|}{r}\right)^{\lambda}
$$

whenever $w_{1}, w_{2} \in B(z, r / c) \cap \hat{D}_{1}$ and $c$ is large enough, depending only on $p, n, \beta_{2}$. With $c^{\prime}$ now fixed we draw the closed line segment $l^{\prime}$ connecting $\eta$ to $\zeta_{1}$. If $y \in l^{\prime}$ let $\Omega^{\prime}(y)$ be the union of all open line segments connecting points in $B\left(y, \frac{1}{8} r / c^{\prime}\right)$ to points in $B\left(z, \frac{1}{8} r / c^{\prime}\right) \cap \partial \hat{D}_{i}, i=1,2$. We claim that

$$
G=\bigcup_{y \in l^{\prime}} \Omega^{\prime}(y) \subset \mathbb{R}^{n} \backslash\left[B\left(z, \frac{1}{8} r / c^{\prime}\right) \cap \partial \Omega_{2}^{+}\right]
$$

so that $\Omega(y), y \in l^{\prime}$, is starlike Lipschitz with respect to any point in $B\left(y, \frac{1}{32} r / c^{\prime}\right)$. Indeed, otherwise we first observe that

$$
L=\bigcup_{y^{\prime} \in l^{\prime}} B\left(y^{\prime}, \frac{1}{8} r / c^{\prime}\right) \subset \mathbb{R}^{n} \backslash\left[B\left(z, \frac{1}{8} r / c^{\prime}\right) \cap \partial \Omega_{2}^{+}\right]
$$

since a ray drawn from a point in $B\left(\eta, \frac{1}{8} r / c^{\prime}\right)$ through a point in $B\left(z, \frac{1}{8} r / c^{\prime}\right) \cap \partial \Omega_{2}^{+}$can never reenter $\hat{D}_{2}$ once it leaves $\hat{D}_{2}$ and $B\left(\zeta_{1}, \frac{1}{8} r / c^{\prime}\right) \subset D_{2}$. Using this observation, we see that if (5.12) is false, then there are points $P, Q, R$ with $P \in L, Q, R \in B\left(z, \frac{1}{8} r / c^{\prime}\right) \cap \partial \hat{\Omega}_{i}^{+}, i=1,2$, such that the line segment $l_{1}$ from $P$ to $Q$ contains $R$. Choose $P_{1}, \in \Omega\left(\zeta_{1}\right)$ and $P_{2} \in \Omega(\eta)$ such that the line segment, $l^{\prime \prime}$, from $P_{1}$ to $P_{2}$ is parallel to $l^{\prime}$ and contains $P$. Let $l_{2}$ be the line segment from $P_{1}$ to $Q$. Then $P_{1}, P_{2}, P, Q, R$ all lie in a two-dimensional plane and $P_{2}$ lies outside the triangle with vertices $P_{1}, P, R$. Draw the infinite ray, $P_{2}$ through $R$. Let $\sigma$ denote the part of this ray 
connecting $R$ to $\infty$. Then by our choice of $\Omega(\eta)$, we must have $\sigma \subset \mathbb{R}^{n} \backslash \hat{D}_{2}$. However clearly $\sigma$ must intersect the open line segment $l^{*}$ from $P_{1}$ to $Q$ and

$$
l^{*} \subset \Omega\left(\zeta_{1}\right) \subset \hat{D}_{1} \cap B(w, 2 r)=\hat{D}_{2} \cap B(w, 2 r)
$$

thanks to our construction of $\Omega\left(\zeta_{1}\right)$ and (5.2). We have reached a contradiction. Thus claim (5.12) is true. From (5.12) we can conclude that $D(y)=\Omega(y) \backslash B\left(y, \frac{1}{16} r / c^{\prime}\right)$ is starlike Lipschitz with center at $\xi$ and constant depending only on $p, n, \beta_{1}, \beta_{2}$, whenever $\xi \in B\left(y, \frac{1}{32} r / c^{\prime}\right)$ and $y \in l^{\prime}$. We can now use Theorem 2 at most $c$ times, as in the proof of (5.9) in order to conclude that

$$
\left|\log \left(\frac{v_{1}\left(w_{1}\right)}{v\left(w_{1}\right)}\right)-\log \left(\frac{v_{1}\left(w_{2}\right)}{v\left(w_{2}\right)}\right)\right| \leqslant c\left(\frac{\left|w_{1}-w_{2}\right|}{r}\right)^{\lambda}
$$

provided $w_{1}, w_{2} \in B(z, r / c) \cap \hat{D}_{1}$ and $c$ is large enough (again depending only on $p, n, \beta_{1}, \beta_{2}$ ). Combining (5.10), (5.11), (5.13), we deduce first that (5.5) is true and thereupon, from our earlier remarks, that Theorem 2 is true when $w$ lies in the unbounded components of $\mathbb{R}^{n} \backslash \hat{D}_{i}, i=1,2$.

Using essentially the same argument we can deduce Theorem 2 when $w$ lies in the bounded components of $\mathbb{R}^{n} \backslash \hat{D}_{i}, i=1,2$. One small change in the proof is to choose $c^{\prime}$ so large that rays drawn from $B\left(\eta, \frac{1}{8} r / c^{\prime}\right)$ through points in $B\left(z, \frac{1}{8} r / c^{\prime}\right) \cap \partial \hat{D}_{2}$ cannot reenter $\hat{D}_{2}$ in $B(w, 2 r)$. Then $\Omega(\eta)=B(w, 2 r) \cap H(\eta) \cap \hat{D}_{2}$ is the union of line segments joining points in $B\left(\eta, \frac{1}{8} r / c^{\prime}\right)$ with $B\left(w, r / c^{\prime}\right) \cap \partial \hat{D}_{2}$ and the proof of (5.13) is unchanged. We omit the rest of the details. The proof of Theorem 2 is now complete.

Proof of Theorem 3. - Let $D$ be the starlike Lipschitz ring domain in Theorem 3 with center $\hat{x}$ and $p$ capacitary function $u$. Let $\mu=\mu^{+}-\mu^{-}$be the measure corresponding to $u$ as in Lemma 2.35. Let $w \in \partial D, r$ be as in (1.12) and recall from Lemma 2.39 that $d \mu / d H^{n-1}=$ $\pm k^{p-1}$ on $B(w, 8 r) \cap D$ where the + sign is taken if $w$ is in the outer boundary of $D$ while the - sign is taken if $w$ lies in the inner boundary of $D$. Moreover, applying Lemma 2.39 with $w, r$ replaced by $y \in B(w, 8 r) \cap \partial D$ and $s$ so that $B(y, 8 s) \subset B(w, 8 r)$, we deduce

$$
\int_{B(y, s) \cap \partial D} k^{p} d H^{n-1} \leqslant c s^{-\frac{n-1}{p-1}}\left(\int_{B(y, s) \cap \partial D} k^{p-1} d H^{n-1}\right)^{p /(p-1)} .
$$

The above inequality and Lemma 2.35(b) imply (see [18]) that for some $q^{\prime}>p$ (depending only on $p, n$ and the Lipschitz constant for $D$ ) that

$$
\int_{B(w, 3 r) \cap \partial D} k^{q^{\prime}} d H^{n-1} \leqslant c r^{-\frac{(n-1)\left(q^{\prime}+1-p\right)}{p-1}}\left(\int_{B(w, 3 r) \cap \partial D} k^{p-1} d H^{n-1}\right)^{q^{\prime} /(p-1)} .
$$

Let $y \in \partial D \cap B(w, 2 r)$ and $z \in \Gamma(y) \cap B(y, r / 4)$, where $\Gamma(y), y \in B(w, 2 r) \cap D$, is defined for a fixed constant $b$ above (1.13). From Lemmas 2.35 and 2.5 with $w, x$ replaced by $y, z$ and $s=|z-y|$, we obtain that

$$
\begin{aligned}
|\nabla u(z)| & \leqslant c \min \{u(z), 1-u(z)\} / s \leqslant c^{2} s^{-1}\left[s^{p-n}|\mu(B(y, s))|\right]^{1 /(p-1)} \\
& =c^{2}\left[s^{1-n} \int_{B(y, s) \cap \partial D} k^{p-1} d H^{n-1}\right]^{1 /(p-1)} \leqslant c^{2}\left[M\left(k^{p-1}\right)(y)\right]^{1 /(p-1)}
\end{aligned}
$$

$4^{\text {e }}$ SÉRIE - TOME $40-2007-\mathrm{N}^{\circ} 5$ 
where

$$
M(f)(y)=\sup _{0<s<r / 4}\left[s^{1-n} \int_{B(y, s)} f d H^{n-1}\right]
$$

whenever $f$ is an integrable function on $\partial D \cap B(w, 4 r)$. Let

$$
N_{1}(|\nabla u|)(y)=\sup _{\Gamma(y) \cap B(y, r / 4)}|\nabla u| .
$$

Then from (5.14), (5.15), and the Hardy-Littlewood maximal theorem, we get for $q=\left(q^{\prime}+p\right) / 2$ that

$$
\begin{aligned}
& \int_{\substack{B(w, 3 r) \cap \partial D \\
6)}} N_{1}(|\nabla u|)^{q} d H^{n-1} \leqslant c \int_{B(w, 3 r) \cap \partial D} M\left(k^{p-1}\right)^{q /(p-1)} d H^{n-1} \\
& \leqslant c^{2} r^{-\frac{(n-1)(q+1-p)}{p-1}}\left(\int_{B(w, 3 r) \cap \partial D} k^{p-1} d H^{n-1}\right)^{q /(p-1)} .
\end{aligned}
$$

Using Lemma 2.3 we see that $|\nabla u(x)| \leqslant c r^{-1}$ when $x \in \Gamma(y) \backslash B(y, r / 2)$ and $y \in \partial D$. Thus $N(|\nabla u|) \leqslant N_{1}(|\nabla u|)+c r^{-1}$ on $\partial D \cap B(w, 2 r)$. In view of (5.16) it now follows that $(a)$ of Theorem 3 is true.

The proof that $\nabla u$ has nontangential limits $H^{n-1}$ almost everywhere is by contradiction. Suppose for a fixed constant, in the definition of $\Gamma(z), z \in \partial D$, that

$$
\lim _{y \in \Gamma(z), y \rightarrow z} \nabla u(y) \text { does not exist for } z \in F \subset \partial D \text { with } H^{n-1}(F)>0 .
$$

Let $w \in F$ be an $H^{n-1}$ point of density for $F$. Then $\lim _{t \rightarrow 0} t^{1-n} H^{n-1}[B(w, t) \cap \partial D \backslash F]=0$, so if $r>0$ is small enough, we deduce that

$$
c H^{n-1}[\partial \tilde{\Omega} \cap B(w, r) \cap F] \geqslant r^{n-1}
$$

where $\tilde{\Omega} \subset D$ is as in Lemma 2.45. From this lemma, we have $|\nabla u| \approx c$ on $\tilde{\Omega}$. Define $\left(b_{i j}(x)\right)$ as in (1.8) with $\hat{u}$ replaced by $u$ and let $L$ be the corresponding divergence form elliptic operator defined as in (1.7). Then as noted above (2.26) we have $L u_{x_{k}}=0$ in $D$ for $1 \leqslant k \leqslant n$. Moreover $L$ is uniformly elliptic on $\tilde{\Omega}$ and $u_{x_{k}}$ is bounded on $\tilde{\Omega}$. It now follows from a well-known argument (see [10]) that $u_{x_{k}}$ has nontangential limits at almost every boundary point of $\tilde{\Omega}$ with respect to elliptic measure defined relative to $L$ (see Section 3). Here $b, 1<b<\infty$, is arbitrary in the choice of nontangential cones for $\tilde{\Omega}$. From Theorem 3.11 we conclude that $u_{x_{k}}$ has nontangential limits at $H^{n-1}$ almost every point in $\partial \tilde{\Omega}$. Since $b$ is arbitrary, and $\partial \tilde{\Omega}$ has tangent planes $H^{n-1}$ a.e., we see that this limit can only exist if (5.17) is false.

Let $\nabla u(w), w \in \partial D$, denote the nontangential limit of $\nabla u$ whenever this limit exists. To prove $(b)$ of Theorem 3 we can retrace the argument leading to Lemma 2.39. Indeed in (2.44) we showed that if $w$ is in the outer boundary of $D, D(t)=\{y \in D: t<u(y)<1\}$, and $t$ is near 0 , then

$$
\int_{B(w, 2 r) \cap\{u=t\}}|\nabla u|^{p} d H^{n-1} \leqslant c r^{-\frac{n-1}{p-1}}\left(\int_{B(w, 2 r) \cap\{u=t\}}|\nabla u|^{p-1} d H^{n-1}\right)^{p /(p-1)} .
$$


If $w$ lies in the inner boundary of $D$, this inequality also holds provided $t$ is near enough 1 . From Lemma 2.5 we see that if $\{x: u(x)=t\}=\left\{R_{t}(\omega) \omega: \omega \in \partial B(0,1)\right\}$, then $\left\{\log R_{t}(\cdot)\right\}$ are uniformly Lipschitz on $\partial B(0,1)$. Using this fact, the fact that $\nabla u$ has radial limits $H^{n-1}$ almost everywhere, Theorem $3(a)$, and dominated convergence, we get that (5.18) holds with $\{u=t\}$ replaced by $\partial D$. Also from (2.38) and Lemma 2.39 we find that

$$
\nu \approx|\mu| \quad \text { where } d \nu=|\nabla u|^{p-1} d H^{n-1} \text { on } \partial D .
$$

Theorem 3(b) now follows from (5.14) and (5.19). Theorem 3(b) implies Theorem 3(c) (see [11]). The proof of Theorem 3 is complete.

Remark. - For use in the proof of Theorem 3 we point out that a slightly more sophisticated argument in Theorem 3 yields,

$$
\nu=|\mu| \quad \text { on } \partial D \cap B(w, 2 r) .
$$

In fact extend $R_{t}$ to $\mathbb{R}^{n} \backslash\{0\}$ by putting $R_{t}(x)=R_{t}\left(\frac{x}{|x|}\right)$. Since $u\left(R_{t}(\omega) \omega\right)=t$ for $\omega \in \partial B(0,1)$, we can use the implicit function theorem and the chain rule to deduce for $\omega=\left(\omega_{1}, \ldots, \omega_{n}\right)$ and $x=|x| \omega$ that

$$
\nabla \log R_{t}(\omega)=-\frac{|x| \nabla u(x)}{\langle x, \nabla u(x)\rangle}+\frac{x}{|x|} .
$$

We note that $|\nabla u| \neq 0, H^{n-1}$ almost everywhere, on $\partial D$ as follows from Theorem 3 and [11]. Using this note, (5.21), Lemma 2.5, and Theorem 3, we see that if $y \in \Gamma(z) \cap\{x: u(x)=t\} \rightarrow$ $z \in \partial D$, then

$$
\nabla \log R_{t}(y /|y|) \rightarrow-\frac{|z| \nabla u(z)}{\langle z, \nabla u(z)\rangle}+\frac{z}{|z|},
$$

$H^{n-1}$ almost everywhere. Let $R$ be a graph function for either the outer or inner boundary of $D$. Since $\log R_{t} \rightarrow \log R$ uniformly on $\partial B(0,1)$ as $t \rightarrow 0$ or 1 , it follows that the right-hand side of (5.22) is a distributional gradient of $\log R$. Thus it is equal $H^{n-1}$ almost everywhere to the gradient of $\log R$ at $z /|z|$. Using this fact, (5.18), (2.38), the change of variables theorem, Lemma 2.5, Theorem 3, and dominated convergence we get (5.20). Finally note that taking limits in (5.22) it also follows that $\nabla u$ is $H^{n-1}$ almost everywhere normal to the tangent planes through points in $\partial D$. Taking limits in (2.41) we conclude that (2.41) holds with $D(t)$ replaced by $D$ where $\nu=\frac{\nabla u}{|\nabla u|}, H^{n-1}$ almost everywhere on $\partial D$.

Proof of Theorem 4. - Let $D, w, r, u, p$ be as in Theorem 3 and suppose also that $\partial D \cap$ $B(w, 8 r)$ is $C^{1}$. To prove Theorem 4 it suffices by way of a lemma of Sarason (see [27]) to show that there exists $\epsilon_{0}>0, r_{0}=r_{0}(\epsilon)$ defined for $\epsilon \in\left(0, \epsilon_{0}\right)$, such that whenever $y \in B(w, r) \cap \partial D$ and $0<s<r_{0}(\epsilon)$, then

$$
f_{B(y, s) \cap \partial D}|\nabla u|^{p} d H^{n-1} \leqslant(1+\epsilon)\left(f_{B(y, s) \cap \partial D}|\nabla u|^{p-1} d H^{n-1}\right)^{p /(p-1)} .
$$

Here

$$
f_{E} f d H^{n-1}=\left[H^{n-1}(E)\right]^{-1} \int_{E} f d H^{n-1}
$$

$4^{\text {e }}$ SÉRIE - TOME $40-2007-\mathrm{N}^{\circ} 5$ 
whenever $E \subset \partial D$ is Borel measurable with finite positive $H^{n-1}$ measure and $f$ is an $H^{n-1}$ integrable function on $E$. The proof of (5.23) is by contradiction. Indeed, otherwise, there exist sequences $\left(s_{m}\right)_{1}^{\infty},\left(y_{m}\right)_{1}^{\infty}$ satisfying $y_{m} \in \partial D \cap B(w, r), m=1,2, \ldots, \lim _{m \rightarrow \infty} s_{m}=0$, and for which (5.23) is false with $y, s$ replaced by $y_{m}, s_{m}, m=1,2, \ldots$. To obtain a contradiction we note from $C^{1}$ smoothness of $\partial D \cap B(w, 8 r)$ that $\partial D \cap B(w, 4 r)$ is Reifenberg flat with vanishing constant (see [27]) in the following sense. Given $\delta>0$, small, there is an $a=a(\delta)<10^{-6} r$, such that whenever $y \in \partial D \cap B(w, 4 r)$ and $0<s \leqslant a$, then

$$
\begin{gathered}
\{z+t \nu \in B(y, s / \delta), z \in P, t>\delta s\} \subset D, \\
\{z-t \nu \in B(y, s / \delta), z \in P, t>\delta s\} \subset \mathbb{R}^{n} \backslash \bar{D} .
\end{gathered}
$$

Here $P=P(y)$ is the tangent plane to $\partial D \cap B(w, 4 r)$ at $y$ and $\nu=\nu(y)$ is the corresponding unit normal pointing into $D$. For fixed $A>10^{6}$ we can also choose $\delta_{0}=\delta_{0}(A)>0$ so small that if $y^{\prime}=y+A s \nu(y)$, then the domain $\Omega\left(y^{\prime}\right)$, obtained by drawing all line segments from points in $B\left(y^{\prime}, \frac{A s}{4}\right)$ to points in $\partial D \cap B(y, A s)$, is starlike Lipschitz with respect to $y^{\prime}$. We assume, as we may, that $s_{m} \leqslant a\left(\delta_{0}\right)$, for $m=1,2, \ldots$ Let $y_{m}^{\prime}$ be the point corresponding to $y_{m}$ as above and put $D_{m}=\Omega\left(y_{m}^{\prime}\right) \backslash \bar{B}\left(y_{m}^{\prime}, A s_{m} / 8\right)$. From $C^{1}$ smoothness of $\partial D \cap B(w, 8 r)$, we see that $D_{m}, m=1,2, \ldots$, has Lipschitz constant $\leqslant c_{0}$ where $c_{0}$ is an absolute constant. Let $u_{m}$ be the $p$ capacitary function for $D_{m}, m=1,2, \ldots$. From Theorem 2 with $r, w, \hat{u}_{1}, \hat{u}_{2}$ replaced by $\frac{A s_{m}}{100}, y_{m}, u, u_{m}$, we deduce that if $w_{1}, w_{2} \in B\left(y_{m}, 2 s_{m}\right) \cap D, m=1,2, \ldots$, then

$$
\left|\log \left(\frac{u_{m}\left(w_{1}\right)}{u\left(w_{1}\right)}\right)-\log \left(\frac{u_{m}\left(w_{2}\right)}{u\left(w_{2}\right)}\right)\right| \leqslant c A^{-\alpha},
$$

where $c, \alpha$ are the constants in Theorem 2, so are independent of $m$. Letting $w_{1}, w_{2} \rightarrow z_{1}, z_{2} \in$ $\partial D \cap B\left(y_{m}, 2 s_{m}\right)$, and using Theorem 3, we get $H^{n-1}$ almost everywhere that

$$
\left|\log \left(\frac{\left|\nabla u_{m}\left(z_{1}\right)\right|}{\left|\nabla u\left(z_{1}\right)\right|}\right)-\log \left(\frac{\left|\nabla u_{m}\left(z_{2}\right)\right|}{\left|\nabla u\left(z_{2}\right)\right|}\right)\right| \leqslant c A^{-\alpha} .
$$

Exponentiating this inequality we have for $c$ large enough,

$$
\left(1-\tilde{c} A^{-\alpha}\right) \frac{\left|\nabla u_{m}\left(z_{1}\right)\right|}{\left|\nabla u_{m}\left(z_{2}\right)\right|} \leqslant \frac{\left|\nabla u\left(z_{1}\right)\right|}{\left|\nabla u\left(z_{2}\right)\right|} \leqslant\left(1+\tilde{c} A^{-\alpha}\right) \frac{\left|\nabla u_{m}\left(z_{1}\right)\right|}{\left|\nabla u_{m}\left(z_{2}\right)\right|},
$$

where $\tilde{c}$ depends only on $p, n$, and the Lipschitz constant for $D$. Using this inequality and the fact that (5.23) is false, we obtain

$$
\begin{aligned}
& \frac{f_{B\left(y_{m}, s_{m}\right) \cap \partial D}\left|\nabla u_{m}\right|^{p} d H^{n-1}}{\left(f_{B\left(y_{m}, s_{m}\right) \cap \partial D}\left|\nabla u_{m}\right|^{p-1} d H^{n-1}\right)^{p /(p-1)}} \\
& \geqslant\left(1-c A^{-\alpha}\right) \frac{f_{B\left(y_{m}, s_{m}\right) \cap \partial D}|\nabla u|^{p} d H^{n-1}}{\left(f_{B\left(y_{m}, s_{m}\right) \cap \partial D}|\nabla u|^{p-1} d H^{n-1}\right)^{p /(p-1)}} \geqslant\left(1-c A^{-\alpha}\right)(1+\epsilon) .
\end{aligned}
$$

Let $T_{m}$ be a conformal affine mapping of $\mathbb{R}^{n}$ which maps the origin, $e_{n}$, onto $y_{m}, y_{m}^{\prime}$ respectively and $W=\left\{x \in \mathbb{R}^{n}: x_{n}=0\right\}$ onto $P\left(y_{m}\right)$ for $m=1,2, \ldots$ It is easily seen that $T_{m}$ is the composition of a translation, rotation, dilation. Let $D_{m}^{\prime}, u_{m}^{\prime}$ be such that $T_{m}\left(D_{m}^{\prime}\right)=D_{m}$ and $u_{m}\left(T_{m} x\right)=u_{m}^{\prime}(x)$ whenever $x \in D_{m}^{\prime}$. Since the $p$ Laplace equation is invariant under 
translations, rotations, and dilations, we see that $u_{m}^{\prime}$ is the $p$ capacitary function for $D_{m}^{\prime}$. From (5.25) we also get

$$
\begin{aligned}
& \frac{f_{B(0,1 / A) \cap \partial D_{m}^{\prime}}\left|\nabla u_{m}^{\prime}\right|^{p} d H^{n-1}}{\left(f_{B(0,1 / A) \cap \partial D_{m}^{\prime}}\left|\nabla u_{m}^{\prime}\right|^{p-1} d H^{n-1}\right)^{p /(p-1)}} \\
& =\frac{f_{B\left(y_{m}, s_{m}\right) \cap \partial D}\left|\nabla u_{m}\right|^{p} d H^{n-1}}{\left(f_{B\left(y_{m}, s_{m}\right) \cap \partial D}\left|\nabla u_{m}\right|^{p-1} d H^{n-1}\right)^{p /(p-1)}} \geqslant\left(1-c A^{-\alpha}\right)(1+\epsilon) .
\end{aligned}
$$

Letting $m \rightarrow \infty$ we see from Lemmas 2.1,2.2 that $u_{m}^{\prime}$ converges uniformly on $\mathbb{R}^{n}$ to $u^{\prime}$ where $u^{\prime}$ is the $p$ capacitary function for the starlike Lipschitz ring domain, $D^{\prime}=\hat{\Omega} \backslash B\left(e_{n}, 1 / 8\right)$. Also $\hat{\Omega}$ is obtained by drawing all line segments connecting points in $B(0,1) \cap W$ to points in $B\left(e_{n}, 1 / 4\right)$. To get a contradiction we shall show that

$$
\begin{gathered}
\limsup _{m \rightarrow \infty}\left[\frac{f_{B(0,1 / A) \cap \partial D_{m}^{\prime}}\left|\nabla u_{m}^{\prime}\right|^{p} d H^{n-1}}{\left(f_{B(0,1 / A) \cap \partial D_{m}^{\prime}}\left|\nabla u_{m}^{\prime}\right|^{p-1} d H^{n-1}\right)^{p /(p-1)}}\right] \\
\leqslant \frac{f_{B(0,1 / A) \cap W}\left|\nabla u^{\prime}\right|^{p} d H^{n-1}}{\left(f_{B(0,1 / A) \cap W}\left|\nabla u^{\prime}\right|^{p-1} d H^{n-1}\right)^{p /(p-1)}}
\end{gathered}
$$

To see how (5.27) leads to a contradiction, note from Schwarz reflection that $u^{\prime}$ has a $p$ harmonic extension to $B(0,1 / 8)$ with $u^{\prime} \equiv 0$ on $W \cap B(0,1 / 8)$. From barrier estimates we have $c^{-1} \leqslant\left|\nabla u^{\prime}\right| \leqslant c$ on $B(0,1 / 16)$ where $c$ depends only on $p, n$, and from Lemma 2.3 we find that $\left|\nabla u^{\prime}\right|$ is Hölder continuous with exponent $\sigma$ on $\bar{B}(0,1 / 16) \cap W$ (one could take $\sigma=1$ here). Using these facts we conclude first that for some $c$

$$
\left(1-c A^{-\sigma}\right)\left|\nabla u^{\prime}(0)\right| \leqslant\left|\nabla u^{\prime}(z)\right| \leqslant\left(1+c A^{-\sigma}\right)\left|\nabla u^{\prime}(0)\right|
$$

when $z \in B(0,1 / A)$ and second from (5.26), (5.27) that

$$
\left(1+c A^{-\sigma}\right) \geqslant \frac{f_{B(0,1 / A) \cap W}\left|\nabla u^{\prime}\right|^{p} d H^{n-1}}{\left(\int_{B(0,1 / A) \cap W}\left|\nabla u^{\prime}\right|^{p-1} d H^{n-1}\right)^{p /(p-1)}} \geqslant\left(1-c A^{-\alpha}\right)(1+\epsilon) .
$$

Clearly this inequality cannot hold if $A=e^{1 / \epsilon}$ and $\epsilon_{0}$ is sufficiently small. From this contradiction we conclude that in order to prove Theorem 4 it suffices to prove (5.27). To prove (5.27) we shall need the following lemma.

LEMMA 5.28. - Given $\eta>0$ small, there exists $c$, depending only on $p, n$, and the Lipschitz constant for $D$ and $m_{0}=m_{0}(\eta)$, such that if $m \geqslant m_{0}, x \in \partial D_{m}^{\prime} \cap B(0,1 / 100)$, and $0 \leqslant s \leqslant$ $1 / 100$, then $u_{m}^{\prime}\left(a_{s}(x)\right) \leqslant c s^{1-\eta}$.

Proof. - Let $\nu_{m}^{\prime}$ be the outer unit normal to $\partial D_{m}^{\prime}$. We note for large $m$ that since $\partial D$ is $C^{1}$, there is a truncated cone $L \subset \mathbb{R}^{n} \backslash D_{m}^{\prime}$ with vertex at $x$, axis parallel to $\nu_{m}^{\prime}(x)$, angle opening $\theta>\pi / 2$, and height $1 / 50$, where $\theta$ can be arbitrarily near $\pi / 2$. Let $T$ be the cone with vertex at the origin, axis parallel to $e_{n}$, and of angle opening $\theta$. Arguing as in [33] we see there exists a positive solution $f=f(\cdot, \theta)$ to the $p$ Laplacian in $T$ of the form

$$
f(z)=|z|^{\gamma} \psi\left(\frac{\left\langle z, e_{n}\right\rangle}{|z|}\right), \quad z \in T
$$

$4^{e}$ SÉRIE - TOME $40-2007-\mathrm{N}^{\circ} 5$ 
where $\psi \in C^{\infty}[\cos \theta, 1]$ and $\psi(1)=1, \psi(\cos \theta)=0$. Using the maximum principle for $p$ harmonic functions, rotation and translation invariance of the $p$ Laplacian, as well as Theorem 1, it follows that the conclusion of Lemma 5.28 holds with $1-\eta$ replaced by $\gamma=\gamma(\theta)$. Thus to complete the proof of Lemma 5.28 it suffices to show that $\gamma(\theta) \rightarrow 1$ continuously as $\theta \rightarrow \pi / 2$. This can be easily verified using a compactness argument, since any convergence subsequence say $\left\{f\left(\cdot, \theta_{j}\right)\right\}$ with $\theta_{j} \rightarrow \pi / 2$ is a positive solution to the $p$ Laplacian on $\left\{x: x_{n}>0\right\}$ which vanishes on $W$. This solution also has the form (5.29) with $\gamma=\lim _{j \rightarrow \infty} \gamma\left(\theta_{j}\right)$. Using Theorem 1 and the fact that $x_{n}$ is also a positive solution to the $p$ Laplacian on $\left\{x: x_{n}>0\right\}$ which vanishes on $W$, it follows that $\gamma=1$.

Another proof of Lemma 5.28 which uses only the fact that $\partial D \cap B(w, 4 r)$ is Reifenberg flat with vanishing constant (see (5.24)) and an iteration argument, can be given as in [36, (2.37)]. The proof in [36] gives a lower bound for $u_{m}^{\prime}\left(a_{s}(x)\right)$ and is only in $\mathbb{R}^{2}$. However thanks to Theorem 1, essentially the same iterative argument can be used to get Lemma 5.28.

Finally we use Lemma 5.28 to prove (5.27). Let $\mu_{m}=\mu_{m}^{+}-\mu_{m}^{-}$, be the measure associated with $u_{m}^{\prime}$ for $m=1,2, \ldots$. From (5.20) we see that

$$
d\left|\mu_{m}\right|=\left|\nabla u_{m}^{\prime}\right|^{p-1} d H^{n-1} \quad \text { on } \partial D_{m}^{\prime} .
$$

From Lemmas 2.1, 2.2, and 2.35 we see that $\left\{\left|\mu_{m}\right|\right\}$ converges weakly to $\left|\mu^{\prime}\right|$ where $\mu^{\prime}=$ $\mu_{\prime}^{+}-\mu_{\prime}^{-}$is the measure associated with $u^{\prime}$. Also (5.30) holds for $\left|\mu_{\prime}\right|, u^{\prime}$. From the definition of weak convergence and (5.30) we deduce

$$
\int_{B(0,1 / A) \cap W}\left|\nabla u^{\prime}\right|^{p-1} d H^{n-1}=\lim _{m \rightarrow \infty} \int_{B(0,1 / A) \cap \partial D_{m}^{\prime}}\left|\nabla u_{m}^{\prime}\right|^{p-1} d H^{n-1} .
$$

Let $C=\left\{x=\left(x^{\prime}, x_{n}\right) \in \mathbb{R}^{n}:\left|x^{\prime}\right|<1 / A,\left|x_{n}\right|<1 / A\right\}$ be the symmetric cylinder of radius $1 / A$, height $2 / A$ with center at the origin and axis parallel to $e_{n}$. Let $\nu^{*}$ be the outer unit normal to $C$. To handle the top integral in (5.27) we argue as in the proof of Lemma 2.39 leading to (2.41). As noted after (5.22), this inequality holds with $u, D(t), B(w, s)$, replaced by $u_{m}^{\prime}, D_{m}^{\prime}, C$, and with $\nabla u_{m}^{\prime}$ as the normal, $H^{n-1}$ almost everywhere, to $\partial D_{m}^{\prime}$. We get

$$
L_{m}=(p-1) \int_{\partial D_{m}^{\prime} \cap C}\left\langle e_{n}-x, \nabla u_{m}^{\prime}\right\rangle\left|\nabla u_{m}^{\prime}\right|^{p-1} d H^{n-1}=J_{m}+K_{m},
$$

where

$$
J_{m}=(p-n) \int_{D_{m}^{\prime} \cap C}\left|\nabla u_{m}^{\prime}\right|^{p} d x
$$

and

$$
\begin{aligned}
K_{m}= & \int_{D_{m}^{\prime} \cap \partial C}\left\langle x-e_{n}, \nu^{*}\right\rangle\left|\nabla u_{m}^{\prime}\right|^{p} d H^{n-1} \\
& -p \int_{D_{m}^{\prime} \cap \partial C}\left\langle\nabla u_{m}^{\prime}, \nu^{*}\right\rangle\left\langle\nabla u_{m}^{\prime}, x-e_{n}\right\rangle\left|\nabla u_{m}^{\prime}\right|^{p-2} d H^{n-1} .
\end{aligned}
$$

Now $u_{m}^{\prime}, \nabla u_{m}^{\prime}$ converge uniformly on compact subsets of $D^{\prime}$ to $u^{\prime}, \nabla u^{\prime}$. Replace $u$ by $u_{m}^{\prime}$ in Theorem 3. From the proof of Theorem 3(a) (see (5.16)) we deduce that the $L^{q}$ norm $(q>p)$ of 
$N\left(\left|\nabla u_{m}^{\prime}\right|\right)$ on $\partial D_{m}^{\prime} \cap B(0,1 / 8)$ is uniformly bounded for $m=1,2, \ldots$ Using these facts and standard convergence theorems we see that

$$
\lim _{m \rightarrow \infty} J_{m}=(p-n) \int_{D^{\prime} \cap C}\left|\nabla u^{\prime}\right|^{p} d x .
$$

The same argument can be applied to $K_{m}$ to get

$$
\begin{aligned}
\lim _{m \rightarrow \infty} K_{m}= & \int_{D^{\prime} \cap \partial C}\left\langle x-e_{n}, \nu^{*}\right\rangle\left|\nabla u^{\prime}\right|^{p} d H^{n-1} \\
& -p \int_{D^{\prime} \cap \partial C}\left\langle\nabla u^{\prime}, \nu^{*}\right\rangle\left\langle\nabla u^{\prime}, x-e_{n}\right\rangle\left|\nabla u^{\prime}\right|^{p-2} d H^{n-1}
\end{aligned}
$$

provided we first show that

$$
\int_{D_{m}^{\prime} \cap \partial C}\left|\nabla u_{m}^{\prime}\right|^{p+1} d H^{n-1} \leqslant M<\infty
$$

for sufficiently large $m$, say $m \geqslant m_{0}$. To prove (5.35) note from our construction that $\partial D_{m}^{\prime} \cap \partial C$ is a Lipschitz image of $\left\{x^{\prime} \in \mathbb{R}^{n-1}:\left|x^{\prime}\right|=1 / A\right\}$, with Lipschitz constant independent of $m$. Let

$$
\Lambda_{k}=\left\{x \in D_{m}^{\prime} \cap \partial C: A^{-1} 2^{-k-1+n}<d\left(x, \partial D_{m}^{\prime}\right)<A^{-1} 2^{-k-n}\right\} \quad \text { for } k=3, \ldots
$$

From Lemma 5.28 and Lemma 2.5(ii) for $u_{m}^{\prime}$, we see that if $m_{0}$ is large enough, then for $m>m_{o}$,

$$
\left|\nabla u_{m}^{\prime}\right|^{p+1} \leqslant c A 2^{k / 2}
$$

on $\Lambda_{k}$ where $c$ is independent of $m$ and $k$. If $\left|x^{\prime}\right|=1 / A$, and $l$ denotes the line through $x^{\prime}$ parallel to $e_{n}$, then from (5.36) and the above note we deduce that

$$
\int_{l \cap \Lambda_{k}}\left|\nabla u_{m}^{\prime}\right|^{p+1}\left(x^{\prime}, \cdot\right) d H^{1} \leqslant c 2^{-k / 2} .
$$

Integrating with respect to $H^{n-2}$ measure over the sphere of radius $1 / A$ in $\mathbb{R}^{n-1}$, it follows that

$$
\int_{\Lambda_{k}}\left|\nabla u_{m}^{\prime}\right|^{p+1} d H^{n-1} \leqslant c 2^{-k / 2} A^{2-n} .
$$

Let $\Lambda=\bigcup_{k=1}^{\infty} \Lambda_{k}$. Summing (5.37) we get

$$
\int_{\Lambda}\left|\nabla u_{m}^{\prime}\right|^{p+1} d H^{n-1} \leqslant c A^{2-n} .
$$

Now from (5.24) we see for $m_{0}$ large enough that $c d\left(C \backslash \Lambda, \partial D_{m}^{\prime}\right) \geqslant A^{-1}$ where $c$ is independent of $m$. From this fact,(5.38), and uniform convergence of $\nabla u_{m}^{\prime}$ to $\nabla u^{\prime}$ on compact subsets of $D^{\prime}$

$4^{\text {e }}$ SÉRIE - TOME $40-2007-\mathrm{N}^{\circ} 5$ 
we find that (5.35) is valid. Thus (5.34) is also valid. Now, (5.32) also holds with $u_{m}^{\prime}$ replaced by $u^{\prime}$ and $\partial D_{m}$ by $\partial D^{\prime}$. In view of (5.33), (5.34) and (5.32) for $u^{\prime}, u_{m}^{\prime}$ we deduce that

$$
\begin{aligned}
\lim _{m \rightarrow \infty} L_{m} & =(p-1) \int_{W \cap B(0,1 / A)}\left\langle e_{n}-x, \nabla u^{\prime}\right\rangle\left|\nabla u^{\prime}\right|^{p-1} d H^{n-1} \\
& =(p-1) \int_{W \cap B(0,1 / A)}\left|\nabla u^{\prime}\right|^{p} d H^{n-1} .
\end{aligned}
$$

Since $\nabla u_{m}^{\prime}(x), x \in \partial D_{m}^{\prime}$, is normal $H^{n-1}$ almost everywhere to the tangent plane through $x$, we deduce from $C^{1}$ smoothness of $\partial D \cap B(w, 8 r)$ and our construction that

$$
|| \nabla u_{m}^{\prime}\left|+\left\langle x-e_{n}, \nabla u_{m}^{\prime}\right\rangle\right| \leqslant b_{m}\left|\nabla u_{m}^{\prime}\right| \quad \text { on } \partial D_{m}^{\prime} \cap C
$$

where $b_{m} \rightarrow 0$ as $m \rightarrow \infty$. Thus,

$$
\lim _{m \rightarrow \infty} L_{m}=(p-1) \lim _{m \rightarrow \infty} \int_{\partial D_{m}^{\prime} \cap C}\left|\nabla u_{m}^{\prime}\right|^{p} d H^{n-1} .
$$

Next we note from $C^{1}$ smoothness of $\partial D \cap B(w, 8 r)$ that

$$
H^{n-1}\left[\partial D_{m}^{\prime} \cap B(0,1 / A)\right] \rightarrow H^{n-1}[W \cap B(0,1 / A)] \quad \text { as } m \rightarrow \infty .
$$

Using (5.40) in (5.39), the fact that $B(0,1 / A) \subset C$, (5.41), and (5.31), we conclude that (5.27) is true. From our earlier remarks, we now get Theorem 4.

\section{Acknowledgements}

Finally the first author would like to thank Nicola Garofalo who challenged him on Theorem 1 nearly 20 years ago and Nages Shanmugalingam for her interest and encouragement in proving Theorem 1.

\section{REFERENCES}

[1] Aikawa H., Shanmugalingam N., Carleson type estimates for $p$ harmonic functions and the conformal Martin boundary of John domains in metric measure spaces, Michigan Math. J. 53 (1) (2005) 165-188.

[2] Aikawa H., Kilpeläinen T., Shanmugalingam N., Zhong X., Boundary Harnack principle for $p$ harmonic functions in smooth Euclidean domains, submitted for publication.

[3] Alt H.W., Caffarelli L., Existence and regularity for a minimum problem with free boundary, J. reine angew. Math. 325 (1981) 105-144.

[4] AnCONA A., Principe de Harnack à la frontière et théorème de Fatou pour un opérateur elliptique dans un domaine lipschitzien, Ann. Inst. Fourier (Grenoble) 28 (4) (1978) 169-213.

[5] Bennewitz B., LeWIS J., On the dimension of $p$ harmonic measure, Ann. Acad. Sci. Fenn. 30 (2005) 459-505.

[6] BENNEWITZ B., LEWIS J., On weak reverse Hölder inequalities for nondoubling harmonic measures, Complex Variables 49 (7-9) (2004) 571-582.

[7] CAfFARELli L., A Harnack inequality approach to the regularity of free boundaries. Part I. Lipschitz free boundaries are $C^{1, \alpha}$, Rev. Math. Iberoamericana 3 (1987) 139-162. 
[8] Caffarelli L., A Harnack inequality approach to the regularity of free boundaries. II. Flat free boundaries are Lipschitz, Comm. Pure Appl. Math. 42 (1) (1989) 55-78.

[9] CAfFARelli L., A Harnack inequality approach to the regularity of free boundaries. III. Existence theory, compactness, and dependence on X, Ann. Scuola Norm. Sup. Pisa Cl. Sci. (4) 15 (4) (1989) 583-602.

[10] Caffarelli L., Fabes E., Mortola S., Salsa S., Boundary behavior of nonnegative solutions of elliptic operators in divergence form, Indiana J. Math. 30 (4) (1981) 621-640.

[11] CoIfMAnN R., FefFerman C., Weighted norm inequalities for maximal functions and singular integrals, Studia Math. 51 (1974) 241-250.

[12] Dahlberg B., On estimates of harmonic measure, Arch. Ration. Mech. Anal. 65 (1977) 275-288.

[13] DiBenedetto E., $C^{1+\alpha}$ local regularity of weak solutions of degenerate elliptic equations, Nonlinear Anal. 7 (1983) 827-850.

[14] Eremenko A., Lewis J., Uniform limits of certain $A$-harmonic functions with applications to quasiregular mappings, Ann. Acad. Sci. Fenn. AI, Math. 16 (1991) 361-375.

[15] FABes E., Kenig C., SERAPIONi R., The local regularity of solutions to degenerate elliptic equations, Comm. Partial Differential Equations 7 (1) (1982) 77-116.

[16] Fabes E., Jerison D., Kenig C., The Wiener test for degenerate elliptic equations, Ann. Inst. Fourier (Grenoble) 32 (3) (1982) 151-182.

[17] FABEs E., JERISON D., KENIG C., Boundary behavior of solutions to degenerate elliptic equations, in: Conference on Harmonic Analysis in Honor of Antonio Zygmund, vols. I, II, Chicago, IL, 1981, in: Wadsworth Math. Ser., Wadsworth, Belmont, CA, 1983, pp. 577-589.

[18] Gehring F., On the $L^{p}$ integrability of the derivatives of a quasiconformal mapping, Acta Math. 130 (1973) 265-277.

[19] GARIEPY R., ZIEMER W.P., A regularity condition at the boundary for solutions of quasilinear elliptic equations, Arch. Ration. Mech. Anal. 67 (1977) 25-39.

[20] Gilbarg D., Trudinger N.S., Elliptic Partial Differential Equations of Second Order, second ed., Springer-Verlag, 1983.

[21] Heinonen J., Kilpeläinen T., Martio O., Nonlinear Potential Theory of Degenerate Elliptic Equations, Oxford University Press, 1993.

[22] Hofmann S., LEWIS J., The Dirichlet problem for parabolic operators with singular drift term, Mem. Amer. Math. Soc. 151 (719) (2001) 1-113.

[23] JERISON D., Regularity of the Poisson kernel and free boundary problems, Colloq. Math. 60-61 (1990) 547-567.

[24] JERISON D., KENIG C., Boundary behavior of harmonic functions in nontangentially accessible domains, Adv. Math. 46 (1982) 80-147.

[25] JeRISON D., Kenig C., The logarithm of the Poisson kernel of a $C^{1}$ domain has vanishing mean oscillation, Trans. Amer. Math. Soc. 273 (1984) 781-794.

[26] KEMPER J., A boundary Harnack inequality for Lipschitz domains and the principle of positive singularities, Comm. Pure Appl. Math. 25 (1972) 247-255.

[27] Kenig C., Toro T., Harmonic measure on locally flat domains, Duke Math. J. 87 (1997) 501-551.

[28] Kenig C., Toro T., Free boundary regularity for harmonic measure and Poisson kernels, Ann. of Math. 150 (1999) 369-454.

[29] Kenig C., Toro T., Poisson kernel characterization of Reifenberg flat chord arc domains, Ann. Sci. Ecole Norm. Sup. (4) 36 (3) (2003) 323-401.

[30] Kenig C., TORo T., Free boundary regularity below the continuous threshold: 2-phase problems, submitted for publication.

[31] Kenig C., Pipher J., The Dirichlet problem for elliptic operators with drift term, Publ. Mat. 45 (1) (2001) 199-217.

[32] KilpeläInen T., Zhong X., Growth of entire $A$-subharmonic functions, Ann. Acad. Sci. Fenn. AI, Math. 28 (2003) 181-192.

[33] KROL' I.N., On the behavior of the solutions of a quasilinear equation near null salient points of the boundary, Proc. Steklov Inst. Math. 125 (1973) 130-136.

[34] Lewis J., Vogel A., Uniqueness in a free boundary problem, Comm. Partial Differential Equations 31 (2006) 1591-1614.

$4^{\text {e }}$ SÉRIE - TOME $40-2007-\mathrm{N}^{\circ} 5$ 
[35] LEWIS J., Vogel A., Symmetry problems and uniform rectifiability, submitted for publication.

[36] LEWIS J., Note on $p$ harmonic measure, Comput. Methods Funct. Theory 6 (1) (2006) 109-144.

[37] LEWIS J., Regularity of the derivatives of solutions to certain degenerate elliptic equations, Indiana Univ. Math. J. 32 (6) (1983) 849-858.

[38] Lieberman G.M., Boundary regularity for solutions of degenerate elliptic equations, Nonlinear Anal. 12 (11) (1988) 1203-1219.

[39] Littman W., Stampacchia G., Weinberger H.F., Regular points for elliptic equations with discontinuous coefficients, Ann. Scuola Norm. Sup. Pisa (3) 17 (1963) 43-77.

[40] Mattila P., Geometry of Sets and Measures in Euclidean Spaces, Cambridge University Press, 1995.

[41] SERRIN J., Local behavior of solutions of quasilinear elliptic equations, Acta Math. 111 (1964) 247302.

[42] STEIN E.M., Singular Integrals and Differentiability Properties of Functions, Princeton University Press, Princeton, NJ, 1970.

[43] TolksdorfF P., Everywhere regularity for some quasi-linear systems with lack of ellipticity, Ann. Math. Pura Appl. 134 (4) (1984) 241-266.

[44] WU J.M., Comparisons of kernel functions, boundary Harnack principle and relative Fatou theorem on Lipschitz domains, Ann. Inst. Fourier (Grenoble) 28 (4) (1978) 147-167.

(Manuscrit reçu le 12 février 2007;

accepté, après révision, le 3 septembre 2007.)

John L. LEWIS

Department of Mathematics, University of Kentucky,

Lexington, KY 40506-0027, USA

E-mail: john@ms.uky.edu

Kaj NYSTRÖM

Department of Mathematics,

Umeå University,

S-90187 Umeå, Sweden

E-mail: kaj.nystrom@math.umu.se 